\title{
The effect of different road load implementation strategies on fuel economy of USPS step vans
}

\author{
Seiar Ahmad Zia \\ West Virginia University
}

Follow this and additional works at: https://researchrepository.wvu.edu/etd

\section{Recommended Citation}

Zia, Seiar Ahmad, "The effect of different road load implementation strategies on fuel economy of USPS step vans" (2009). Graduate Theses, Dissertations, and Problem Reports. 4556.

https://researchrepository.wvu.edu/etd/4556

This Thesis is protected by copyright and/or related rights. It has been brought to you by the The Research Repository @ WVU with permission from the rights-holder(s). You are free to use this Thesis in any way that is permitted by the copyright and related rights legislation that applies to your use. For other uses you must obtain permission from the rights-holder(s) directly, unless additional rights are indicated by a Creative Commons license in the record and/ or on the work itself. This Thesis has been accepted for inclusion in WVU Graduate Theses, Dissertations, and Problem Reports collection by an authorized administrator of The Research Repository @ WVU. For more information, please contact researchrepository@mail.wvu.edu. 


\title{
The Effect of Different Road Load Implementation Strategies on Fuel Economy of USPS Step Vans
}

\author{
Seiar Ahmad Zia \\ Thesis submitted to the \\ College of Engineering and Mineral Resources \\ At West Virginia University \\ In partial fulfillment of the requirements \\ for the degree of \\ Master of Science \\ in \\ Mechanical Engineering \\ Benjamin C. Shade, Ph.D.. Chair \\ Mridul Gautam, Ph. D. \\ Nigel N. Clark, Ph. D. \\ Department of Mechanical and Aerospace Engineering
}

\author{
Morgantown, West Virginia
}

2009

Keywords: Hybrids, Chassis Testing, Road Load, Coastdowns, Fuel Economy, State of Charge 


\title{
ABSTRACT \\ The Effect of Different Road Load Implementation Strategies on Fuel Economy of USPS Step Vans
}

\author{
Seiar Ahmad Zia
}

West Virginia University (WVU) is continuously improving and updating their testing quality, procedures and goals. As tests are conducted on the Transportable Heavy Duty Vehicle Emissions Testing Laboratory (THDVETL), real world implementation is crucial in order to compare and contrast vehicles within fleets, use of exhaust aftertreatment devices and hybridized vehicles. WVU implements road loads on the chassis dynamometer using a method described in 40 CFR $\S 86.1229-85$. The proposed method requires three variables from the vehicle: maximum height, maximum width and vehicle weight. The issue with this method arises due to the fact that it does not cover a wide range of heavy duty vehicle physical characteristics.

An alternative form of implementing road loads is to conduct on-road coastdowns and use regression analysis to determine the vehicles' characteristics such as coefficient of drag and coefficient of rolling resistance. The coastdown procedure involves driving a vehicle to a speed and setting the transmission to neutral and letting the vehicle slow until it reaches a complete stop. Since there is no power being transmitted to the wheels, regression analysis of the speed versus time can be used to determine physical characteristics of the vehicle. Using the road load equation, which consists of four components (hill climbing load, inertial load, aerodynamic resistance and rolling resistance) one can implement real world power demand on the chassis dynamometer.

20 tests were conducted using the FTP-75 test schedule and two USPS step vans with one being a hybridized version. Four test configurations were used for this study, loaded and unloaded for each of the two vehicles. The empirical method of road load implementation proved to be more suitable for this USPS step van compared to the theoretical method. The theoretical method assumes that the vehicle's aerodynamic drag is 0.735 compared to the empirical method's equates to 0.669 . WVU uses eddy current dynamometers as a power absorption system to simulate aerodynamic drag and rolling resistance. The power absorption setting for the theoretical model was higher than that of empirical model, as expected. A noticeable fuel economy comparison for both vehicles arose due to more aggressive setting from the theoretical method compared to that of empirical method. The hybrid vehicle showed a $34.4 \%$ better fuel economy compared to the baseline vehicle using the empirical method. The theoretical method showed an improvement of $24.8 \%$ from the hybrid vehicle compared to the baseline vehicle. Comparing the amount of work done for each vehicle during the test cycles, the theoretical method showed a $2.29 \%$ difference between the two vehicles compared to the empirical method of $15.0 \%$. This study proves that the theoretical model forces the hybrid vehicle to operate at higher loads where the full potential of the system is not used. 


\section{ACKNOWLEDGEMENTS}

I have never thought of ever reaching this far in my life after the experiences I have been through. Going to graduate school was one my best choices I have ever made in my collegiate career and my life.

I would never have gotten through engineering with help of my colleagues, friends, advisors, and my family. I first would like to thank Dr. Benjamin Shade for taking a chance with me and giving me the opportunity to prove myself. I have learned a great deal by working with him closely in the laboratory and being involved in technical sessions. I would like to thank Daniel Carder as well, who introduced me to the USPS project and gave me the opportunity to the study on these vehicles.

I would also like to thank my committee members Dr. Gautam and Dr. Clark for the support that have given me. Dr. Gautam, who introduced to Thomas Spencer and encouraged me to get involved in the field of engines and emissions in my undergraduate years, and Dr. Clark, who has been patient with me as I have interrupted him many times in his office to ask him a question regarding hybrid vehicles and systems.

I had the opportunity to work with many other people on this study and other exciting studies: Richard Atkinson, Zac Luzader, Jason England, Chris Rowe, Neil Buzzerd, Bradley Ralston, Ron Jerrette, Curtis Leasor.

Throughout my life, I have had the love and support of the most wonderful family in the world. Thank you Mom and Dad for teaching me the value of hard work and motivating me to excel myself. The rest of my family, my brother and sister, my relatives in Virginia and Netherlands for having played a huge role in my life achievements. Thank you everyone! 


\section{TABLE OF CONTENTS}

ABSTRACT _ ii

ACKNOWLEDGEMENTS __ iii

TABLE OF CONTENTS ___ iv

LIST OF EQUATIONS __ viii

LIST OF FIGURES __ ix

NOMENCLATURE __ xi

1. Introduction

1.1. Goal _ـ 1

1.2. Objectives___ 2

2. Literature Review __ 3

2.1. Hybrid Vehicles and Fuel Economy __ 4

2.1.1. Hino Motors _ـ 4

2.1.2. Idaho National Laboratory ___ 4

2.1.3. Organization for the Promotion of Low Emission Vehicles (LEVO)

2.1.4. West Virginia University ___ 7

2.1.5. West Virginia University __ 8

2.2. Chassis Testing and Road Load Implementation___ 9

2.2.1. West Virginia University ___ 9

2.2.2. National Renewable Energy Laboratory___ 10

2.2.3. General Motors _ 10

2.2.4. University of Maryland____ 11

2.2.5. Loughborough University____ 11

2.3. Drag __ 12

2.3.1. University of Maryland___ 12

2.3.2. University of Illinois ___ 13

2.3.3. The Motor Industry Research Association ___ 14

2.4. Rolling Resistance ___ 14

2.4.1. General Motors __ 14

2.4.2. Jet Propulsion Laboratory ___ 15 
2.4.3. GM Road Surface Study ___ 15

3. Experimental Setup __ 17

4. Procedure __ 26

4.1. Empirical and Theoretical Model___ 26

4.1.1. Road Load Equation ___ 26

4.1.2. Empirical Model__ 28

4.1.3. Theoretical Model__ 29

4.2. Vehicle Preparations and Coastdowns Procedure ___ 30

4.2.1. Vehicle Installation and Instrumentation __ 33

4.2.2. Vehicle Coastdown on Chassis Dynamometer and Determination of Parasitic

Losses 33

4.2.3. FTP -75 Test Procedure and State of Charge Determination 35

5. Results _ 37

5.1. Coastdown _ 37

5.1.1. On-road Coastdowns___ 37

5.1.2. Evaluation of Coastdowns ___ 43

5.1.3. Empirical and Theoretical Model Results___ 46

5.1.4. Evaluation of Chassis Coastdowns Results ___ 48

5.2. Test Cycle Verification __ 51

5.2.1. FTP Cycles _ـ 51

5.2.2. State of Charge Results___ 53

5.3. Power Absorber Settings Based on the Method of Coastdown __ 54

5.3.1. Hybrid Vehicle __ 54

5.3.2. Baseline Vehicle ___ 69

5.4. Fuel Economy and Work __ 73

6. Conclusion and Recommendations __ 77

6.1. Conclusion _ 77

6.2. Recommendations _ 78

7. References _ 79

8. Appendix __ 82 


\section{LIST OF TABLES}

Table 1. The Basic metrics of the FTP-75 test cycle _ 22

Table 2. Test matrix of the weights and weight of the flywheels used__ 27

Table 3. The characteristics of the coastdown curves__ 46

Table 4. Road load power required at $50 \mathrm{mph} \ldots 47$

Table 5. The results of the Theoretical $\mathrm{C}_{\mathrm{d}}$ and $\mathrm{C}_{\mathrm{rr}}$ based on the EPA model __ 48

Table 6. The FTP-75 test cycle verification for the baseline vehicle ___ 52

Table 7. The FTP-75 test cycle verification for the hybrid vehicle _ 52

Table 8. Summary of all the runs for the hybrid vehicle and the determination of the SOC _ 53

Table 9. The PA setting for the hybrid vehicle during the FTP-75 test schedule using method of

theoretical loaded coastdown __ 54

Table 10. The PA setting for the hybrid vehicle during the FTP-75 test schedule using method of

empirical loaded coastdown _ 54

Table 11. The PA setting for the hybrid vehicle during the FTP-75 test schedule using method of theoretical unladen coastdown 68

Table 12. The PA setting for the baseline vehicle during the FTP-75 test schedule using method of theoretical loaded coastdown __ 69

Table 13. The PA setting for the baseline vehicle during the FTP-75 test schedule using method of empirical loaded coastdown __ 69

Table 14. The PA settings for the baseline vehicle during the FTP-75 test schedule using method of theoretical unladen coastdown __ 71

Table 15. The PA setting for the baseline vehicle during the FTP-75 test schedule using method of empirical unladed coastdown __ 71

Table 16. Summary of the average fuel economy for both methods and vehicles ___ 74 Table 17. Fuel economy comparisons of the loaded vehicles with different methods of road load implementation $\quad 75$

Table 18. Summary of work per distance traveled for both methods and vehicles. ___ 75

Table 19. Comparison of the Vehicles with different coastdown method under loaded condition.

Table 20. Eaton Hybrid Vehicle Description___ 82

Table 21. Baseline (stock) Vehicle Description __ 83

Table 22. Fuel specification from local station___ 87

Table 23. The coefficient of regression from analysis of speed data from FTP-75 test schedule using the method of theoretical unladen coastdown__ 87

Table 24. The coefficient of regression from analysis of speed data from FTP-75 test schedule using the method of theoretical loaded coastdown__ 87

Table 25. The coefficient of regression from analysis of speed data from FTP-75 test schedule using the method of empirical loaded coastdown 
Table 26. The coefficient of regression from analysis of speed data from FTP-75 test schedule using the empirical unladed coastdown

Table 27. The coefficient of regression from analysis of speed data from FTP-75 test schedule using the method of empirical loaded coastdown 88

Table 28. The coefficient of regression from the analysis of speed data from FTP-75 test schedule using the method of theoretical loaded coastdown 88

Table 29. The coefficient of regression from the analysis of speed data from FTP-75 test schedule using the method of theoretical unladen coastdown 88

Table 30. The coefficient of regression from the analysis of power data from the FTP-75 test schedule using the method of theoretical unladed coastdown 88 Table 31. The coefficient of regression from the analysis of power data from the FTP-75 test schedule using the method of theoretical loaded coastdown Table 32. The coefficient of regression from the analysis of power data from the FTP-75 test schedule using the method of empirical coastdown 88

Table 33. The coefficient of regression from the analysis of power data from the FTP-75 test schedule using the method of theoretical loaded coastdown

Table 34. The coefficient of regression from the analysis of power data from the FTP-75 test schedule using the method of empirical loaded coastdown

Table 35. The coefficient of regression from the analysis of power data from the FTP-75 test schedule theoretical unladed coastdown

Table 36. The coefficient of regression from the analysis of power data from the FTP-75 test schedule using the method of empirical unladed coastdown 89

Table 37. FTP-75 test results from the baseline vehicle using the loaded empirical coastdown 90 Table 38. FTP-75 test results from the baseline vehicle using the unladen empirical coastdown 90 Table 39. FTP-75 test results from the baseline vehicle using the loaded theoretical coastdown 90 Table 40. FTP-75 test results from the baseline vehicle using the unladen theoretical coastdown

Table 41. FTP-75 test results from the hybrid vehicle using the loaded empirical coastdown _ 91 Table 42. FTP-75 test results from the hybrid vehicle using the unladen theoretical coastdown 91 Table 43. FTP-75 results from the hybrid vehicle using the loaded theoretical coastdown 92 


\section{LIST OF EQUATIONS}

Equation 1. Test weight determination for the vehicles ................................................... 24

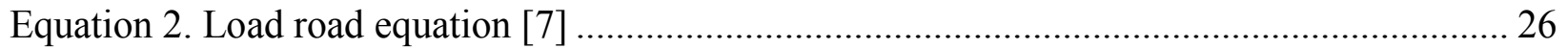

Equation 3. Regression analysis of the deceleration curve ................................................. 28

Equation 4. Regression analysis of deceleration versus speed ............................................... 29

Equation 5. Road load equation as provided by the 40 CFR part 86 subpart M [36]................. 30

Equation 6. Parasitic losses of the chassis dynamometer ..................................................... 34

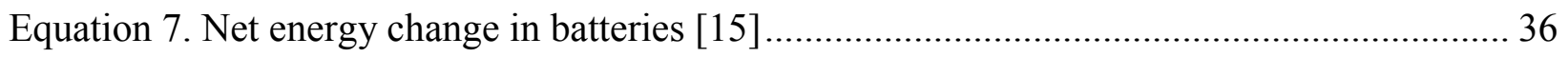

Equation 8. Fuel Energy consumed by the vehicle [15] .................................................... 36

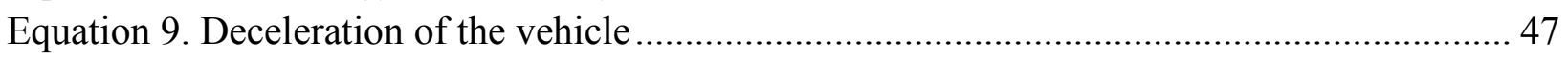




\section{LIST OF FIGURES}

Figure 1. WVU's medium duty chassis dynamometer with a Hybrid USPS Step Van installed at WVU's Westover Laboratory

Figure 2. WVU's 2007 analytical trailer utilizing EPA 40 CFR Part 1065 regulation at WVU's

Westover Laboratory 18

Figure 3. One of the two USPS step van step employed on the chassis dynamometer 19

Figure 4. Four of the six components of the medium duty chassis dynamometer 21

Figure 5. The other two components of the medium duty chassis dynamometer 21

Figure 6. FTP-75 chassis cycle that was exercised for the comparison of coastdowns ___ 22

Figure 7. GPS unit used for the on road coastdowns 24

Figure 8. PA-43, which stretches about 10 miles, is where the coastdown tests were conducted [30] 31

Figure 9. Elevation profile of the road where the coastdowns were conducted [31]

Figure 10. A set of data from the GPS unit during the hybrid vehicle Coastdown on PA-43_ 37

Figure 11. Set of data from the GPS during the unladen baseline vehicle coastdown ___ 38

Figure 12. Set of data from the GPS during the loaded baseline vehicle emission _ 1

Figure 13. The statistical information about the average coastdowns of both vehicles 41

Figure 14. The comparison the loaded coastdown and unladen coastdown using the baseline vehicle 42

Figure 15. The comparison of the average coastdowns with regression analysis 43

Figure 16. The deceleration of all the vehicles with respect to speed 44 Figure 17. The regression analysis of the loaded versus the unladen baseline vehicle coastdowns.

Figure 18. Comparison of the chassis coastdown runs for the hybrid vehicle 49

Figure 19. Comparison of chassis coastdown runs for the baseline vehicle 49

Figure 20. The comparison of the on-road coastdown versus coastdowns conducted on chassis dynamometer

Figure 21. The average of PA settings and the speeds of hybrid vehicle with loaded theoretical method during FTP-75 test schedule, and no motor power was needed during idle periods. 66 Figure 22. The average of the PA settings and the speeds of the hybrid vehicle with loaded empirical method during FTP-75 test schedule, and no motor power were needed during idle periods

Figure 23. The average of the PA settings and speeds of hybrid vehicle with Curb theoretical method, no added motor power was required during idling periods 68 Figure 24. The average of the PA settings and speeds of baseline vehicle with loaded empirical method, no added motor power was required during idling periods 70 Figure 25. The average of the PA settings and speeds of baseline vehicle with loaded theoretical method, no added motor power was required during idling periods 70 
Figure 26. The average of the PA settings and speeds of baseline vehicle with unladen theoretical method during FTP-75 test schedule 72

Figure 27. The average of the PA settings and speeds of baseline vehicle with unladen empirical

method during FTP-75 test schedule 72

Figure 28. Fuel economy comparison of both vehicles for each configuration 74

Figure 29. Work per distance comparison for both vehicles for all four configurations 76

Figure 30. Coastdown comparisons of the hybrid vehicle 84

Figure 31. The elevation change for the hybrid vehicle coastdowns 84

Figure 32. Coastdown comparisons of the unladen baseline vehicle 85

Figure 33. Elevation change for the unladen baseline vehicle for three coastdowns runs 85

Figure 34. Coastdown comparisons of the loaded baseline vehicle 86

Figure 35. Elevation change for the loaded baseline vehicle for the four runs 86 
NOMENCLATURE

a - constants from on-road coastdowns

A - frontal area of the vehicle

ABS - Anti Brake System

AVTA - Advance Vehicle Testing Activity

$\mathrm{b}$ - constants from the deceleration versus speed curve

CAFEE - Center for Alternative Fuels, Engines and Emissions

CAN - computer area network

$\mathrm{C}_{\mathrm{d}}-$ Drag Coefficient

$\mathrm{CO}$ - Carbon Monoxide

$\mathrm{CO}_{2}-$ Carbon Dioxide

$\mathrm{COV}$ - coefficient of variation

$\mathrm{C}_{\mathrm{rr}}-$ Rolling Resistance Coefficient

$\mathrm{F}-$ Factor for Road Load Equations; $\mathrm{F}=1$ for tractor trailer, $\mathrm{F}=.085$ for buses

FTP-75 - Federal Test Procedure 75

g - acceleration due to gravity

GPS - Global Positioning System

GVWR - gross vehicle weight rating

$\mathrm{H}$ - Maximum height

HC - Hydrocarbons

HHDDT - Heavy-Heavy Duty Diesel Truck

$\mathrm{HP}$ - horsepower

JPL - Jet Propulsion Laboratory

LEVO - Promotion of Low Emissions Vehicles

LNG - Liquefied Natural Gas

LVW - Loaded Vehicle Weight

$\mathrm{M}$ - gravimetric mass of the vehicle

MPG - miles per gallon

MPH - Miles Per Hour

MPS - Meters Per Second

NEC - Net Energy Change

$\mathrm{NO}_{\mathrm{x}}$ - Oxides of Nitrogen

NREL - National Renewable Energy Laboratory

PM - Particulate Matter

RESS - Rechargeable Energy Storage System

RLP - Road Load Power in Horsepower

SAE - Society of Automotive Engineers

SG - specific gravity

$\sin \theta$ - road grade

SOC - State of Charge

Std - standard deviation

$\mathrm{t}$ - time

T- torque

USPS - United States Postal Service 
V - velocity

W - Maximum width

whp - wheel horsepower

whp/hr mi - wheel horsepower per hour mile

WVU - West Virginia University

$\omega$ - angular speed

$P$ - power

$\rho$ - density of air 


\section{Introduction}

Chassis dynamometer testing has been developed to evaluate exhaust emissions and fuel economy based on a test schedule developed to simulate vehicles' behavior on the road. West Virginia University (WVU) Center for Alternative Fuels, Engines and Emissions (CAFEE) has specialized in chassis testing over the past decade with many projects [1-5] on heavy duty vehicles using their Transportable Heavy Duty Vehicle Emissions Testing Laboratory (THDVETL) which employs a chassis dynamometer capable of transient vehicle operations. The benefits of using the THDVETL for emissions testing are repeatability of the test schedule and providing a cost effective means of generating emissions data for customers to use for United States regulation [6]. Other benefits of the THDVETL are local businesses' inventory checks and verification of aftertreatment devices and retrofits. In order to implement real world road loads on chassis dynamometers, power absorber settings must be computed for simulation which involves using the road load equation [7]. The unknowns for the road load equation can be determined from either theoretical or empirical methods.

\subsection{Goal}

The primary goal of this study is to compare and contrast an empirical method of coastdowns to a theoretical method through the Federal Test Procedure 75 cycle using WVU's THDVETL and two United States Postal Service (USPS 2-ton) medium duty trucks. The theoretical model, which was the primary method of determining road loads for WVU, involves using a model developed by the Environmental Protection Agency (EPA). The alternative 
method uses the on-road coastdowns and regression analysis to determine the road load characteristics.

\subsection{Objectives}

The primary objective of this study was to show how empirical coastdowns can be more representative of determining the road load constants for chassis testing than the theoretical method that is implemented by EPA. This study could have been done with just a traditional medium duty diesel vehicle, but the addition of a hybrid diesel vehicle will provide another set of data to compare for repeatability of the different coastdown methods.

In addition to investigating the different coastdown methods, another objective was to compare the performance and fuel economy of the baseline vehicle to that of a hybrid vehicle. The work done by each vehicle should be similar, but with hybridization of one vehicle equates to better fuel economy. The proper implementation of road loads is crucial in comparing a hybridized vehicle to a baseline vehicle through chassis dynamometer testing.

Lastly, this study will see how an aggressive power absorber setting influences the performance of the hybrid vehicle and fuel economy. The settings of the power absorbers are determined by the coastdowns. 


\section{Literature Review}

In order to verify road load conditions, the past literature of fuel economy and actual on road behavior of vehicles during chassis testing were examined. With the price and demand for fuel increasing, fuel economy is one of the three main concerns of the engine manufacturers. The other two concerns are reduction of Oxides of Nitrogen $\left(\mathrm{NO}_{\mathrm{x}}\right)$ and Particulate Matter $(\mathrm{PM})[8$, 9]. One way to improve fuel economy is to reduce a vehicle's transient power usage during driving and operate at average power during the demand period. Reduction of power during transient demand can be accomplished by integrating a vehicle with a hybrid system $[2,10]$. During the period of acceleration the hybrid propulsion system would drive the vehicle while the internal combustion engine would be the secondary propulsions system. Numerous papers have been published showing the benefits of hybridization of vehicles in improving fuel economy and reduction of emissions. The results of these publications will be discussed in more detail in the following subsections of this thesis.

In order to investigate fuel economy and emissions from a fleet of vehicles, a test schedule must be used that is best representative of the actual vehicle usage. CAFEE specializes in vehicle data logging and developing cycles that are based on the vehicle's driving behavior during its operation $[3,4,11]$. Data logging involves monitoring parameters such as speed, fueling, throttle position from the ECU and/or instrumented hardware on the vehicle. 


\subsection{Hybrid Vehicles and Fuel Economy}

\subsubsection{Hino Motors}

Hino Motors of Japan successfully developed light duty hybrid trucks that showed three significant achievements: reduction in emissions of $\mathrm{NO}_{\mathrm{x}}$, reduction in emissions of $\mathrm{PM}$, and increased fuel economy. Hino Motor's Hybrid Vehicle Development Division completely redesigned their first light duty hybrid truck with the goal of reducing the size of components of hybrid systems and reducing costs. Hino recognized parallel hybrids as the most cost effective design for development due to the attracted attention from transportation and grocery store commercial businesses. The addition of hybrid vehicles can benefit a truck fleet that has a route with many delivery stops. According to Hino, the design of parallel systems is simple with the engine being the main contributor of propulsion for the vehicle and the hybrid propulsion being mainly used for taking off and accelerating. The fuel consumption of the vehicle decreases while the hybrid system operates during transient and high torque operations, and the diesel engine operates at lower speeds. In conclusion, Hino Motors achieved fuel economy improvements of $18 \%$ to $48 \%$, which were evaluated using various assessment patterns. Concurrently, $\mathrm{NO}_{\mathrm{x}}$ emissions were reduced by $10 \%$ and PM was reduced by $50 \%$. These achievements come from multiple sources such as regenerative control, motor assist control, engine control, weight reduction, and optimization of the overall hybrid system over the course of the evaluation [12].

\subsubsection{Idaho National Laboratory}

US Department of Energy's Advance Vehicle Testing Activity (AVTA) conducted tests baseline performance and fuel economy on a fleet of hybrid passenger and small duty trucks from 2004 to 2006 accumulating 1.4 million miles between 28 hybrid electric vehicles. Even 
though most of the vehicles tested in this study were non-diesel heavy duty vehicles, the study presented a detailed explanation of various methods of fuel economy testing, battery life cycle testing, fleet maintenance, and repair cycle costs. The performances of non-hybrid vehicles were equivalent to the performances of hybridized vehicles of the same model. Some classes of vehicles were designed to maximize performance and some vehicles, such as the Toyota Prius and the Honda Insight, were primarily concentrating on increasing the fuel economy. AVTA also demonstrated the significant impact of air conditioning usage on fuel economy. Fuel economy decreased an average of $21 \%$ when air conditioning was in use at the maximum during the SAE J1634 tests. SAE J1634 technical procedure was used for fuel economy testing. Hybrid electric vehicle repair and maintenance increased considerably as the vehicle progressively aged according to AVTA. There were no correlations between battery degradation and fuel economy loss during battery cycle testing. The Toyota Prius demonstrated a $61.6 \%$ reduction in the battery pack's capacity to hold charge. This decreased the fuel economy by $1.3 \%$. AVTA advised that the $1.3 \%$ change in fuel economy could also be affected by drive train efficiency, driver variability, and sample size of testing. Similar hypotheses can be stated about heavy duty vehicle's battery performance affecting the fuel economy efficiency [13].

\subsubsection{Organization for the Promotion of Low Emission Vehicles (LEVO)}

Takada and et al. [10] investigated fuel economy and $\mathrm{NO}_{\mathrm{x}}$ emissions from light duty hybrid truck in actual traffic conditions. The significance of this study was that it showed correlation among two comparisons consisting of four different factors: fuel economy of the baseline vehicle vs. fuel economy of the hybrid vehicle, and $\mathrm{NO}_{\mathrm{x}}$ emissions of the baseline vehicle vs. $\mathrm{NO}_{\mathrm{x}}$ emissions of the hybrid vehicle. Another factor that was evaluated was the payload of the vehicle over the course of the testing. The hybrid vehicle was a 2003 Hino 2 ton 
grocery delivery truck with a parallel hybrid system consisting of motor/generator, control unit, and battery storage system. The baseline model of this truck did not exist, so a similarly designed vehicle was used to model baseline performance and emissions. The test route consisted of four sections: suburban trip 1, highway trip, urban trip, and suburban trip 2. The total distance the route covered was about 51.8 kilometers and the average speed of the tests was between 22.3 $\mathrm{km} / \mathrm{hr}$ to $29.6 \mathrm{~km} / \mathrm{hr}$. The variation of the average speed was caused by the traffic conditions among the tests. Payloads were set to full, half, and light loading to see how payloads influence fuel economy and $\mathrm{NO}_{\mathrm{x}}$ emissions for the hybrid vehicle. The results of fuel economy testing showed an improvement of $20 \%$ to $40 \%$ during the urban trip and both of the suburban trips compared to the baseline truck. There were no improvements in fuel economy on the highway for the hybrid vehicle compared to the baseline vehicle. The emission of $\mathrm{NO}_{\mathrm{x}}$ improved by $30 \%$ to $40 \%$ compared to the baseline truck. $\mathrm{NO}_{\mathrm{x}}$ improvements include the effect of differences in engine specification, engine control strategy of the hybrid, and the idling stop mechanism. $\mathrm{NO}_{\mathrm{x}}$ emissions was proportional to vehicle payload during the urban trips and both suburban trips, and fuel economy was inversely proportional for trips. Fuel economy and $\mathrm{NO}_{\mathrm{x}}$ emission showed to be constant for the highway trip for the payloads. In the end, the hybrid vehicle demonstrated improvements in fuel economy and $\mathrm{NO}_{\mathrm{x}}$ emissions for the urban and both suburban trips which had utilized many stop-and-go modes. The hybrid vehicle showed to be ineffective during the highway trip and it can be concluded not to be cost effective for the truck fleets. 


\subsubsection{West Virginia University}

West Virginia University THDVETL was used to characterize emissions from a diesel hybrid-powered transit bus, a conventional-drive diesel-powered transit bus, and liquefied natural gas (LNG)-powered transit buses. This program utilized three vehicles for testing: a series-drive diesel hybrid-electric transit bus, a conventional-drive diesel-powered transit bus, and a conventional-drive LNG-powered transit bus. This study followed SAE J2711 Recommended Practice for testing hybrid-electric vehicles for buses. SAE J2711 recommended using the Manhattan cycle, the Orange County Transit Authority cycle (OCTA), and the Urban Dynamometer Driving Schedule (UDDS) presenting low-speed operations, intermediate-speed operations, and high-speed operations, respectively. This study also emphasized the use of longer test cycles, because a single drive cycle is unlikely to affect the state of charge (SOC) at a level sufficient to cause the engine management system to provide additional power to the rechargeable energy storage system (RESS). The use of longer test cycles increases the probability of a smaller net energy change (NEC). SAE J2711 recommended using test cycles around 30 minutes in length. Through the use of statistics, all schedules were modified to fit SAE J2711 criteria. All three vehicles were prepared in accordance to CFR Title 40, Part 86 Subpart N, SAE J2711, and WVU THDVETL standard operating procedures. For road load simulation, WVU used the coastdown experimental method to determine aerodynamics of the vehicle and rolling resistance of the tires. For SOC correction, SAE J2711 recommends determining the energy used by the vehicle from fuel and storage devices. If the \% NEC over the cycle is less than $1 \%$, then there is no need for SOC correction. If the $\%$ NEC over the cycle is between $1 \%$ and 5\%, then SAE J2711 outlines the procedure for emissions and fuel economy correction. And if the $\%$ change is greater than $5 \%$, the test is considered invalid and must be repeated. A significant amount of motive energy is stored onboard the vehicle within RESS and the vehicle 
may remove or add to the energy in the reservoir, depending on the duty cycle. To compare the emissions and fuel economy between hybrid vehicles and conventional vehicles, the data must be corrected so that the NEC in the RESS is essentially zero. In conclusion, the hybrid-electric bus showed the potential to reduce $\mathrm{NO}_{\mathrm{x}}$ emissions by $50 \%$ compared to the conventional bus and by $10 \%$ compared to the LNG powered bus. PM was reduced by $90 \%$, on average, over the different test cycles performed compared to the conventional bus. It was recognized that the hybrid bus was equipped with a catalyzed particulate filter, whereas the conventional diesel bus was equipped with an oxidation catalyst. The hybrid bus showed an average carbon monoxide (CO) reduction of $70 \%$ over the four test cycles and hydrocarbon $(\mathrm{HC})$ reduction of $98 \%$ compared to the conventional diesel bus. There was no fuel economy improvement from the hybrid bus compared to the conventional bus, as was expected $[14,15]$.

\subsubsection{West Virginia University}

West Virginia University THDVETL was used to compare five Lockheed Martin-Orion Hybrid-diesel buses to conventional buses. The vehicles were exercised through three cycles: the Manhattan cycle, the Central Business District cycle, and the New York Bus cycle. The significance of this project was oriented towards the importance of SOC correction and emissions benefits of regeneration. WVU showed comparison of actual emissions produced by the energy used to propel the vehicle to the baseline vehicle. This study used similar laboratory procedures and methods as a previous WVU study [5]. For hybrid testing, WVU used SAE J1711 procedures for testing the hybrid vehicles and even though these procedures are for light duty vehicles, they include similar practices for SOC correction as SAE J2711. The data show that the emissions of $\mathrm{NO}_{\mathrm{x}}$ and $\mathrm{PM}$ were reduced. $\mathrm{NO}_{\mathrm{x}}$ emissions were reduced from $18 \%$ to $40 \%$ depending on the cycle and fuel utilized. Carbon balance was utilized in this study for 
measurement of fuel economy. $\mathrm{NO}_{\mathrm{x}}, \mathrm{CO}_{2}$, and battery $\mathrm{SOC}$ were graphed versus time to show the how they are correlated to each other and why SOC correction is required $[2,15]$.

\subsection{Chassis Testing and Road Load Implementation}

\subsubsection{West Virginia University}

WVU developed a heavy-heavy duty diesel truck (HHDDT) test schedule from speedtime data gathered from 171 heavy duty trucks. The original schedule was considered too aggressive for direct application for HHDDT emissions characterization on a chassis dynamometer. Most class 8 vehicles were determined through prior testing to be unable to follow the original schedule on a chassis dynamometer due to excessive acceleration and deceleration. The new test schedule that was developed used the data from the 171 heavy duty trucks which had produced 1,600 hours or 5.8 million records which was gathered by a global positioning system (GPS). The researcher's development analysis and methodology used HHDDT trips and microtrips. A trip is defined as engine key on and key off and microtrip is defined as stop-to-stop vehicle travel. The results from the data analysis revealed distinct multi-mode patterns of operation defined as idle, creep, transient, and cruise modes and were then reflected in the schedule development [4]. Following preliminary testing, the researchers adopted a test protocol that set the time between each mode to 10 minutes and contained specific driver instructions. The filtered data reduced acceleration and deceleration rate by approximately $50 \%$. 


\subsubsection{National Renewable Energy Laboratory}

The National Renewable Energy Laboratory (NREL) in conjunction with Oshkosh Truck Corporation developed metrics for evaluating duty cycles from a vehicle's energy usage. This study is significant in that it compared hybrid and non-hybrid energy usage independent of the vehicles being used since the equations are derived from the road load equation. By deriving the energy equation from vehicle motion, three applications were introduced. First, metrics were developed to compare and contrast duty cycles from an energy standpoint for their similarities and applicability for hybrid vehicle usage. Second, an equation was formulated from the metrics of the duty cycles to determine the fuel consumption of the vehicle over the target application. The last application includes using a metric developed to check predictions and comparison of hybrid advantage based on how non-propulsion fuel consumption compares to fuel for thrust force of the vehicle [11].

\subsubsection{General Motors}

General Motors implemented a theoretical basis of coastdown testing for establishing the dynamometer loads which simulate the vehicle road loads during fuel economy and emissions testing. Correction for the effects of the ambient conditions on road is also defined in this project. This study was approached by defining the form of the major forces acting on a car as a function of speed with some assumptions, including a level road, steady winds, wind speed being less then vehicle speed, and aerodynamic yaw angles remaining small. Through a series of testing and analyses, it was confirmed that wind is the major source of variance in coastdown data. And it was shown in this study that reducing wind effects on the vehicle can enhance repeatability of the tests. Correction for ambient conditions also significantly reduced the variance in the road test results. These ambient conditions include temperature, barometric pressure, and wind. In the 
end, dynamometer absorber settings were placed to test vehicles with analytically derived variables and it was proven that this was a feasible approach to using coastdowns for road loads [16].

\subsubsection{University of Maryland}

The University of Maryland addressed the problem of fuel consumption with the study of a heavy duty vehicle's aerodynamic drag. A data reduction procedure was presented and successfully used to analyze coastdown data obtained in a windy environment to provide a measure of the aerodynamic drag of a full scale heavy duty vehicle as a function of the yaw angle of the vehicle. The variable being changed in this study is the aerodynamic drag using drag reducing equipment on the vehicle and using coastdown data to produce a drag coefficient. Using coefficients from coastdowns, which is governed by the equation of motion, aerodynamic drag was determined for drag reducing equipment. The highest drag reduction achieved by this process was $32 \%$ at $0^{\circ}$ yaw and $21 \%$ reduction in wind [17].

\subsubsection{Loughborough University}

Loughborough University conducted a detailed drag study using the coastdown method. The difference between this project and that of WVU [14] is that Loughborough University first used a wind tunnel testing to determine coefficient of drag $\left(\mathrm{C}_{\mathrm{d}}\right)$ and it also derived an equation to account for mechanical losses. These mechanical losses included tire losses through rolling resistance $\left(\mathrm{C}_{\mathrm{rr}}\right)$, drivetrain losses, and un-drive tire losses. An anemometer was mounted on the vehicle to establish a time history of relative wind speed and direction. The author stressed the fact that the ambient condition showed to have influence in this analysis just as it was in publication [17]. Through the mathematical model, the author described the aerodynamic drag 
and mechanical drag and determined the total drag as a function of velocity of the vehicle. Four unknown coefficients arise with this final mathematical model of total drag and determinations of these coefficients are the object of this study. For the coastdown, the vehicle was driven at 80 $\mathrm{km} / \mathrm{hr}$ for 20 minutes to warm up the engine and other components such as the tires, transmission, and differential oil. For the results, the $\mathrm{C}_{\mathrm{d}}$ from the experimental method was $7.5 \%$ higher than that of the wind tunnel testing. To have a $C_{d}$ within $1 \%$, the researcher had determined that the user must run 80 coastdown tests. With the 20 tests that were conducted in this study, the accuracy of the four coefficients at the $95 \%$ confidence level were $2.5 \%, 10.8 \%$, $1.9 \%$, and $47 \%$. The $47 \%$ accuracy is originated from the variation of $C_{d}$ from yaw angle. $A$ significant note of this paper is the relatively large losses from tires due to change in inflation pressure [18].

\subsection{Drag}

\subsubsection{University of Maryland}

The University of Maryland conducted a study in which the aerodynamic drags of class 8 heavy duty vehicles were determined. This is the same procedure used for publication in SAE technical procedure in [17] in order to compare the $C_{d}$ from different drag reducing equipment. This article describes three different test method selections for determining aerodynamic drag forces acting on heavy duty trucks. One test method, which has been previously described, is coastdowns. The other two test methods, hill-rolling and constant speed tests, were both found to have more drawbacks than benefits. The vehicle chosen for this experiment was baselined in a wind tunnel in a previous program with three different configurations for drag. Drag reducing 
equipment was designed by the University of Maryland to change the drag configuration for the vehicles. Through a theoretical procedure, equations for all the drag forces were determined. Using coastdowns, anemometers and a few other types of equipment, the constants were determined for the theoretical equations. The results of this study will be correlated in another publication [18] with full scale wind-tunnel tests [19].

\subsubsection{University of Illinois}

The University of Illinois, in conjunction with GM Corporation, Chrysler Corporation, Firestone Corporation, and Goodyear Tire Corporation, evaluated vehicle drag contribution from coastdown tests. The evaluation method was based on the mathematical analysis of a simplified dynamic model which not only allows the separation of aerodynamic and rolling resistance forces, but also utilizes the closed mathematical form of the solution to eliminate the need for differentiating an experimentally determined data curve. A key note stated in this study is the significance of rolling resistance as a function of speed, and affirming that the $\mathrm{C}_{\mathrm{rr}}$ is distinctly constant up to $70 \mathrm{mph}$ for conventional tires, but increases by $53 \%$ if it exceeds that speed. It also provides an illustrated comparison of coastdown tests with variation in tire inflation pressures and stating how it could decrease coastdown time. This decrease in coastdown time could affect $\mathrm{C}_{\mathrm{d}}$ if $\mathrm{C}_{\mathrm{rr}}$ was noted as constant in calculations. The University of Illinois method of determining drag data yielded good results, but cannot be fully considered as a standard method of determining $\mathrm{C}_{\mathrm{rr}}$ due to some large discrepancy in the data for some runs compared to other sources [20]. 


\subsubsection{The Motor Industry Research Association}

White developed an early stage of determining aerodynamic drag of passenger vehicles based on nine feature categories and rating numbers for each category. This method was not intended to be a substitute for wind tunnel testing, but to provide guidelines for the graphic designer so that they can avoid undesirable body features in the early stages of design. Even though this method seems to be a crude way of estimating $C_{d}$, the publication shows results with an accuracy of $\pm 7 \%$ compared to wind tunnel values. The method first takes a vehicle and divides it into six zones and some zones are divided into subzones. For example, the front would be considered zone one and the subzones of one are the outline plan and elevation. All of the zone and subzones add up to a total of nine categories. This method can be the first stage of determining if the $\mathrm{C}_{\mathrm{d}}$ derived from a coastdown method is realistic if there are not wind tunnel data for the vehicle being tested [21].

\subsection{Rolling Resistance}

\subsubsection{General Motors}

Two different departments from GM investigated the energy losses from $\mathrm{C}_{\mathrm{rr}}$ from vehicle tires. They approached this problem by using a simple model of the tire touching a flat ground and applying Newton's Second Law. The uncomplicated model did not depict the magnitude of the rolling resistance to a high level of accuracy; however, the general purpose of this study was simply to expose the basic physics of the $\mathrm{C}_{\mathrm{rr}}$. The amount of energy lost from force required to overcome $\mathrm{C}_{\mathrm{rr}}$ was determined through mathematical calculation. The expression of the simple model was shown as the square of the axle speed with the assumption that the parameters are 
characteristic of a given tire. It was concluded from the theoretical analysis that any of the following characteristics could decrease $\mathrm{C}_{\mathrm{rr}}$ : increasing diameter of the tire, lowering the tread mass, increasing the tire pressure or tire stiffness and/or decreasing the load which deceases footprint length, and also decreasing elasticity of the tire [22].

\subsubsection{Jet Propulsion Laboratory}

The Jet Propulsion Laboratory (JPL) conducted a study to determining $\mathrm{C}_{\mathrm{rr}}$ from coastdown tests. It emphasizes the actuality that most of the resistive force is generated from aerodynamic drag and tire rolling resistance, but also notes other mechanical resistances. The report draws attention to the need for understanding other mechanical losses and shows the theoretical calculation for determining these values for an accurate value of $\mathrm{C}_{\mathrm{rr}}$. The resistive

forces from mechanical losses are considered negligible at low speeds, but drastically increase at higher speeds. In the results, large emphasis was placed on the preparation of the test for accurate results. Characteristic information about the tire deemed to be more important than the $\mathrm{C}_{\mathrm{rr}}$ that was provided by other sources even if it was the same tire. Firestone Tires' calibration done on a tire showed to have different $\mathrm{C}_{\mathrm{rr}}$ according to JPL. Drum calibration performed by Firestone Tires to determine $\mathrm{C}_{\mathrm{rr}}$ agreed quantitatively with that achieved by the coastdown tests of JPL [23].

\subsubsection{GM Road Surface Study}

GM investigated the effects of road surface textures on tire $\mathrm{C}_{\mathrm{rr}}$ in great detail with both indoor testing and outdoor testing. Data obtained from laboratory tire dynamometer tests and outdoor tests conducted on various paved public type roads indicate that losses associated with tire rolling resistance increase as road surface texture increases. As aforementioned in [21, 22], 
$\mathrm{C}_{\mathrm{rr}}$ is based on the characteristic of the tire, but in this publication the characteristic of the tire was divided into four categories. One category is tire design which includes the type of tire, such as bias or radial, as well as material for that design. Another category is tire operating parameters such as load, speed, inflation pressure, steer torque inputs, and driver habit or tire cycle. The third category is ambient conditions, such as temperature and pressure, around the tire. The last category is highway design which generally includes the construction of the road material: gravel, concrete, or asphalt. The results of the laboratory and outdoor tests showed that the rolling resistance of the tire increases with increasing surface texture. The increase in rolling resistance varies from $5 \%$ to $30 \%$ based on the environment, road surface, and tire type. The study emphasized the need for more testing of individual categories to correlate laboratory testing with real world performance. [24]. 


\section{Experimental Setup}

In order to compare fuel economy and coastdowns for the two USPS step vans, repeatability and accuracy of the tests are crucial. WVU's new 2007 transportable laboratory in conjunction with a medium duty chassis dynamometer was used, shown in Figure 1 and Figure 2 , to verify the findings of the two different methods of implementing road loads. One of the main benefits of using chassis dynamometer testing over on-road testing is the repeatability of the results. Numerous studies done by WVU have shown the many benefits of chassis dynamometer testing over the course of last decade [1 - 4]. The new trailer utilizes state of the art equipment and it has been designed to comply with the EPA's new 40 CFR Part 1065 regulation. The new 40 CFR Part 1065 regulation mandates stricter testing standards compared to the 40 CFR Part 86 [26]. Many aspects of the new EPA 40 CFR Part 1065 regulations, as well as the new technologies in the 2007 theoretical laboratory, will not be covered in this report because they do not pertain to the goals of this report. An ASME technical paper written by Wu et al. covers numerous aspects of the 2007 theoretical laboratory including structural design, gaseous emissions, PM emissions collecting [27]. 


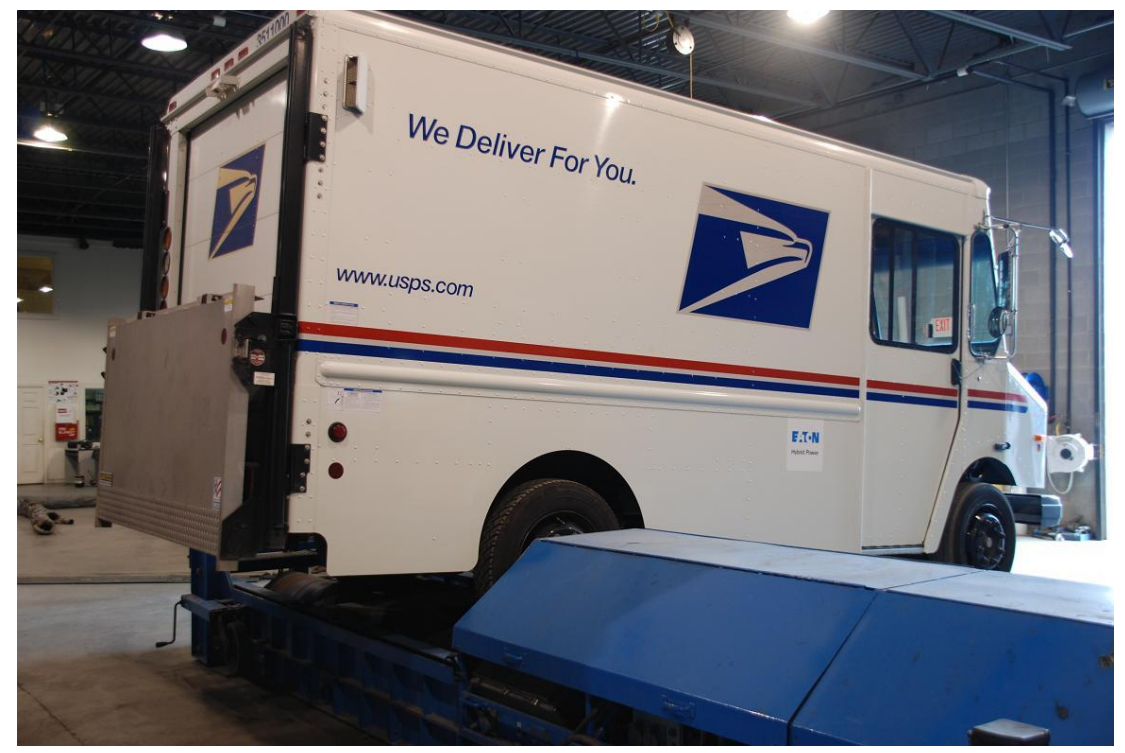

Figure 1. WVU's medium duty chassis dynamometer with a Hybrid USPS Step Van installed at WVU's Westover Laboratory

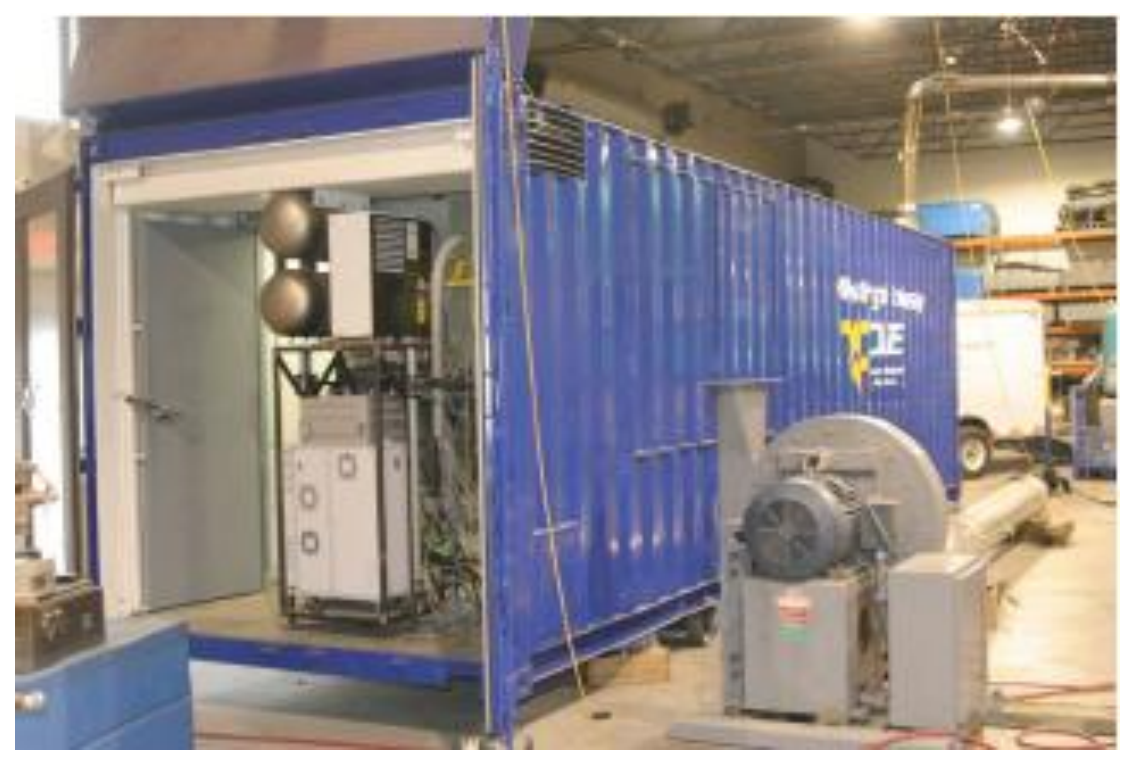

Figure 2. WVU's 2007 analytical trailer utilizing EPA 40 CFR Part 1065 regulation at WVU's Westover Laboratory

The vehicles used for characterization of fuel economy and performance from various coastdown techniques were USPS step vans. Figure 3 below illustrates the vehicle employed on 
a medium duty dynamometer. The exterior dimensions of the hybrid vehicle were exactly the same as the baseline (stock) vehicle. A stock vehicle was retrofitted with hybrid components that added $600 \mathrm{lbs}$ of weight to measured curb weight. The Hybrid USPS step van utilized an Eaton hybrid pre transmission parallel configuration. Due proprietary design of the hybrid system, WVU was not provided specification of the hybrid system by Eaton. Both vehicles were powered by 2003 Mercedes Benz 904 diesel engines. More detailed specifications of the vehicles are shown in Table 20 and Table 21 in the appendixes.

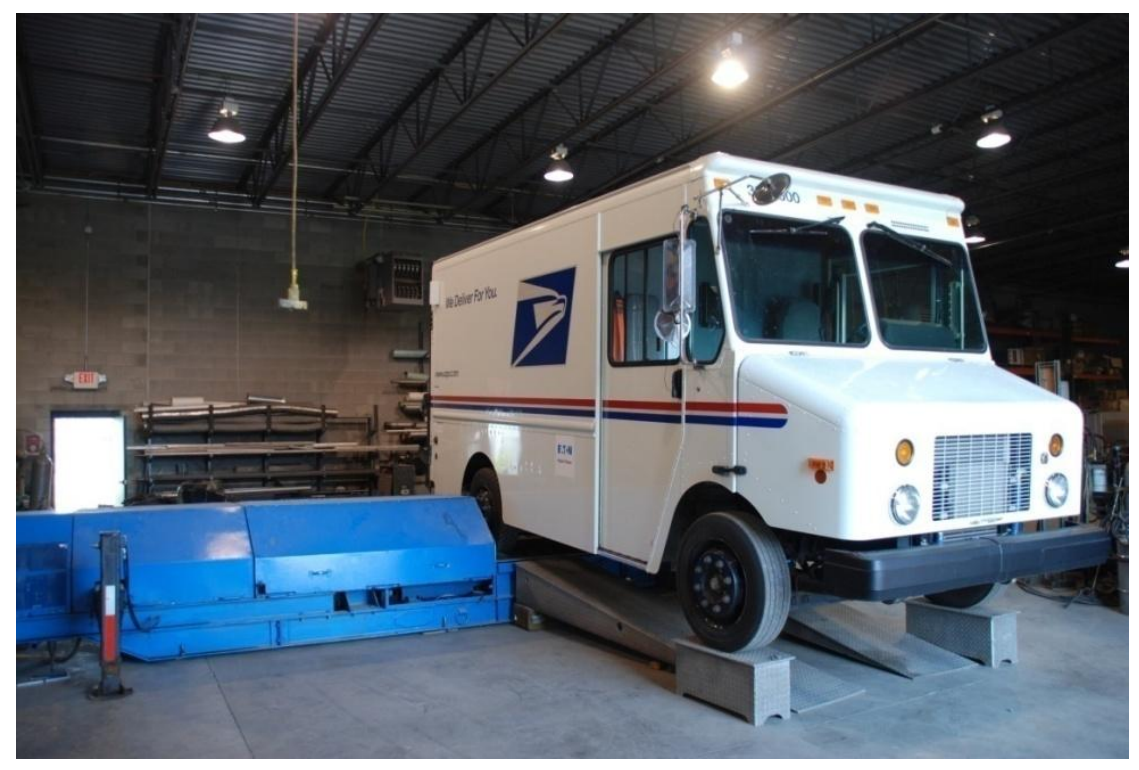

Figure 3. One of the two USPS step van step employed on the chassis dynamometer

For the measurement system, WVU's new analytical laboratory was utilized for these tests. The data acquisition system in the laboratory, developed by WVU, was constructed with the National Instrument SCXI 1001 data acquisition system and the Dyne System's Dyn-Loc IV. A Dyn-Loc IV digital dynamometer controller was used to control the dynamometer 
components. Dyn-Loc IV communicates to SCXI 1001 data acquisition system through a serial adapter which provides feedback from the dynamometer.

A medium duty chassis dynamometer was employed with the new analytical trailer to simulate road loads and vehicle weights. The medium duty chassis dynamometer is capable of simulating the vehicle weights ranging from 3,000 lbs to roughly $22,000 \mathrm{lbs}$. The medium duty chassis dynamometer is comprised of six main components: inertia flywheels, power absorber, two-speed transfer case, AC variable speed motor, inline torque transducer, and tire rollers. Figure 4 and Figure 5 illustrates the six major components of the dynamometer. The design of WVU`s chassis dynamometer has been described in previously published papers [28, 29].

The transfer case provides flexibility in having wider range of speeds. The two possible ratios for speed are 1:1 or 2.04:1. The $25 \mathrm{HP}$ AC motor was used to overcome drivetrain losses of the chassis dynamometer and keep the rollers moving during coasting periods of the test. A SAE technical paper by Wang et al. discusses calculations of the road load as well the losses from the mechanical system of the heavy duty chassis dynamometer. The technical paper uses Newton's second law to determine the mathematical model for the WVU transportable heavy duty testing laboratory which can also be applied to the medium duty testing laboratory. Through the various arrangements of the flywheels, a wide of range of inertial load can be simulated in conjunction with the power absorbers. The inertial flywheels and the power absorbers are part of the power absorption system which simulates vehicle road load for a given speed [29]. 


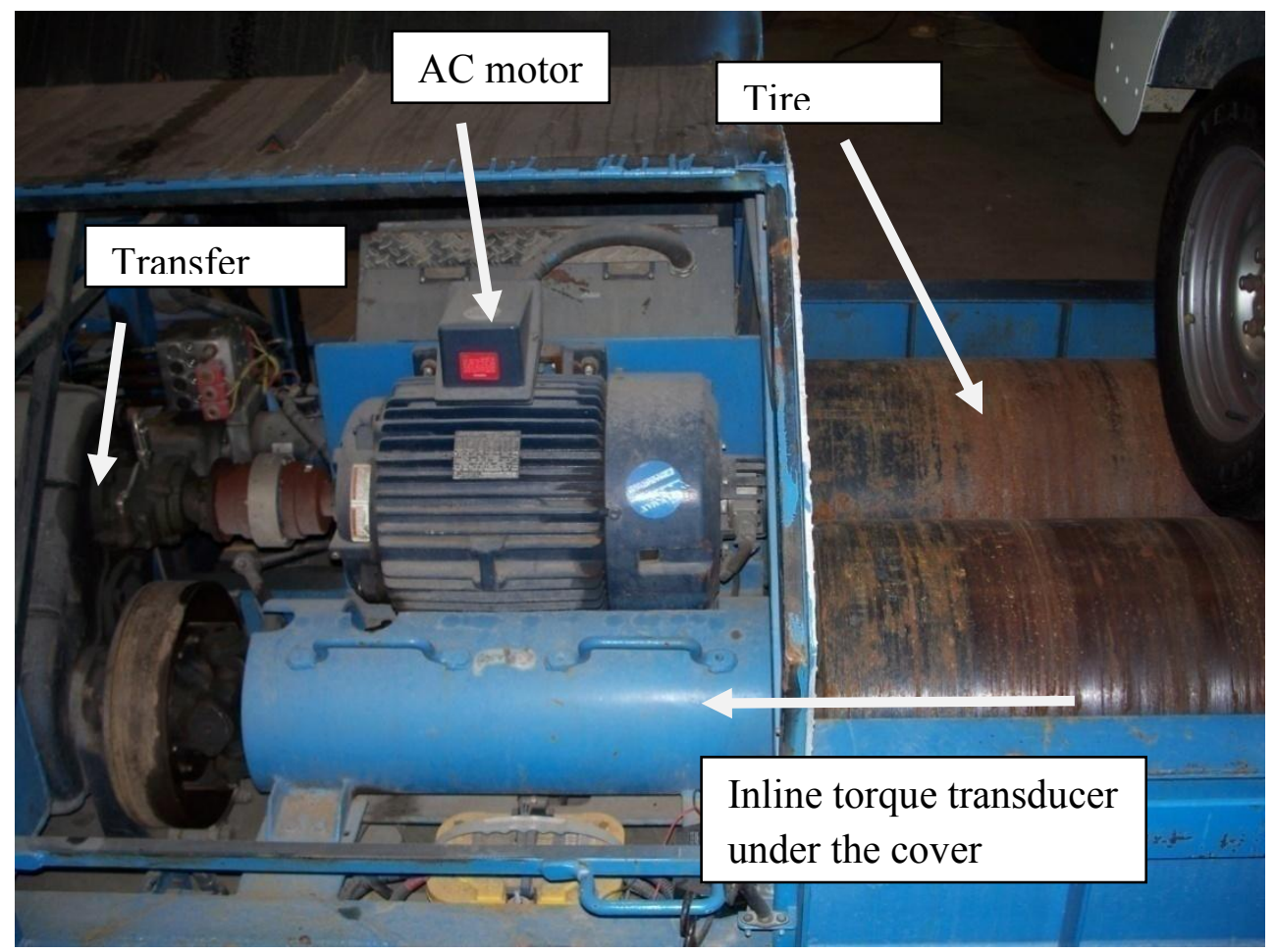

Figure 4. Four of the six components of the medium duty chassis dynamometer

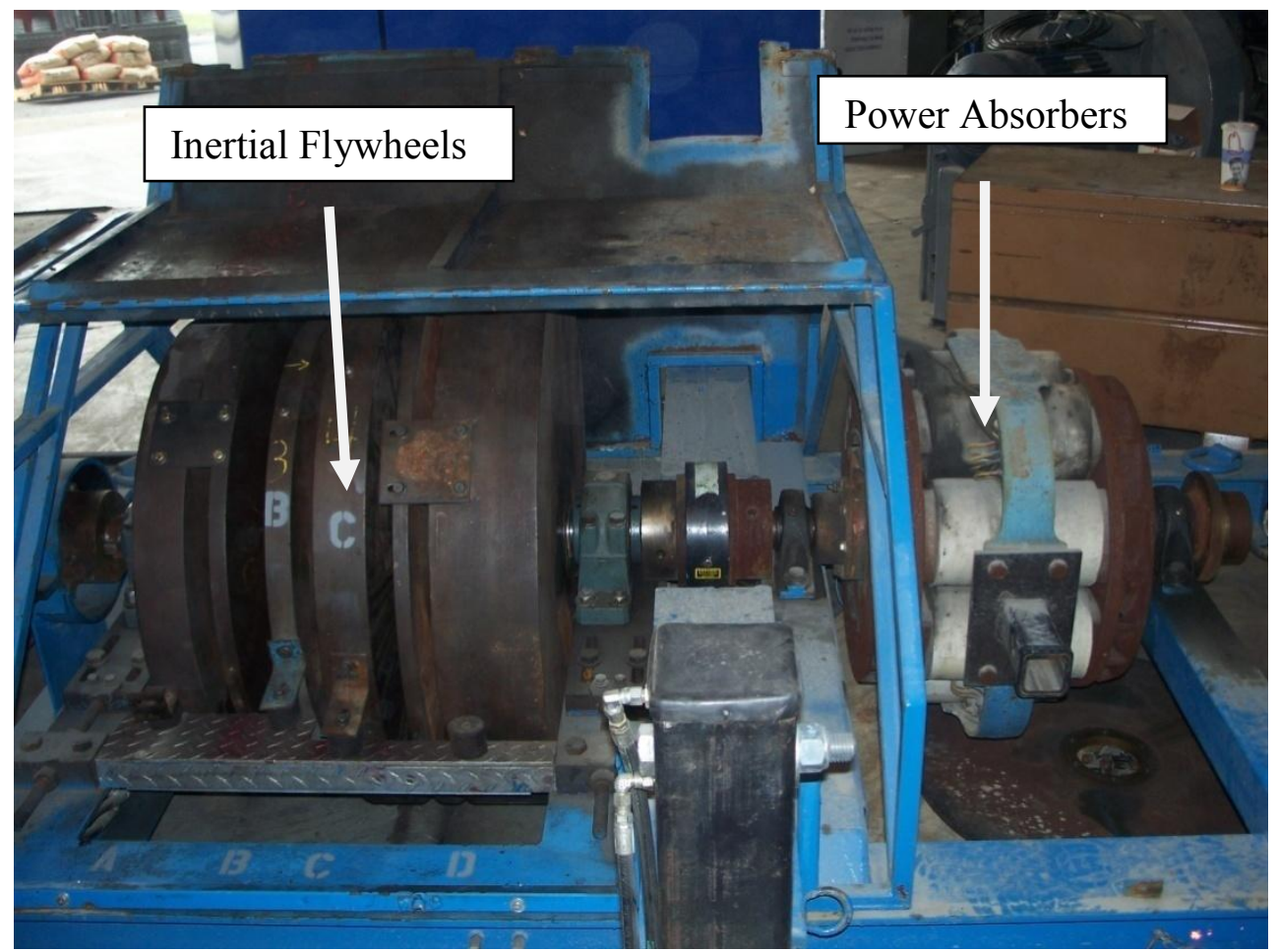

Figure 5. The other two components of the medium duty chassis dynamometer 
The FTP-75 cycle was exercised on the medium duty chassis dynamometer for fuel economy and coastdowns comparison. Figure 6 below shows the FTP-75 test cycle, which has been used for emission certification of light duty vehicles in the United States [25]. The length of the test cycle is 1,874 seconds and covers a distance of 11.04 miles. The transient cycle simulates typical traffic conditions with excessive acceleration and deceleration. A basic summary of the cycle is provided below in the Table 1. The FTP-75 may not be the ideal cycle for the hybrid USPS step van for evaluation of fuel economy, but the idea for having two different vehicles was to show unbiased results from a single vehicle on a set of coastdowns. Recommended cycles for testing hybrid vehicles, according to SAE J2711, are the Manhattan cycle, the UDDS driving cycle, Orange county cycle, or Central Business District cycle [15].

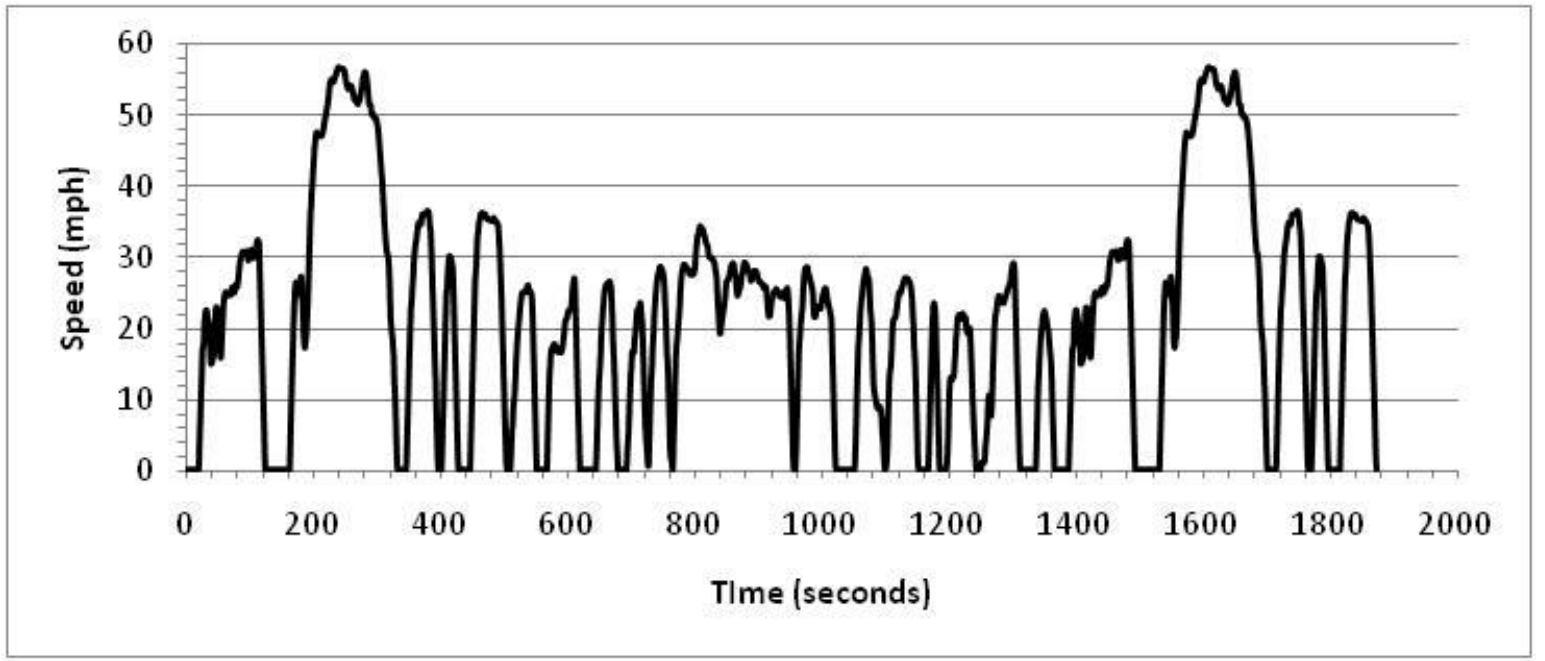

Figure 6. FTP-75 chassis cycle that was exercised for the comparison of coastdowns

Table 1. The Basic metrics of the FTP-75 test cycle

\begin{tabular}{|c|c|c|c|c|c|c|}
\hline $\begin{array}{l}\text { Duratio } \\
\mathrm{n}\end{array}$ & $\begin{array}{l}\text { Average } \\
\text { Speed }\end{array}$ & $\begin{array}{l}\text { Max } \\
\text { Speed }\end{array}$ & $\begin{array}{l}\text { Max } \\
\text { Deceleration }\end{array}$ & $\begin{array}{l}\text { Max } \\
\text { Acceleration }\end{array}$ & $\begin{array}{l}\text { Idle } \\
\text { Period }\end{array}$ & $\begin{array}{l}\text { Distance } \\
\text { Traveled }\end{array}$ \\
\hline (s) & (mph) & (mph) & $(\mathrm{mph} / \mathrm{s})$ & $(\mathrm{mph} / \mathrm{s})$ & (s) & (miles) \\
\hline 1874 & 21.2 & 56.7 & 3.3 & 3.3 & 356 & 11.04 \\
\hline
\end{tabular}


Fuel economy was monitored using the gravimetric method. For the gravimetric method, an Ohaus CD-11 scale was used for the fuel weight. The gravimetric fuel measurement system provided the best accuracy and simplicity. The fuel used for this project was from a local retail fueling station and the specification of the fuel is provided in Table 22 of the appendixes. The commercial ultra low sulfur fuel was stored in a 30-gallon stainless steel drum on the Ohaus scale. The vehicles' fuel lines were disconnected from the storage tank and rerouted to the stainless steel drum. One of the features of the Ohaus CD-11 scale, a RS232 serial connecter, was used to log fuel weight during a test.

For on road coastdown evaluation, the speed of the vehicle must be monitored and recorded during each test. GPS was one method of recording vehicle speed during the coastdown. The GPS system was comprised of three components as shown in Figure 7. The main rectangular box housed the main system of the GPS with two connections. The first connection was for a roof-mounted sensor, which gathered data (at 1 Hertz) from satellites. The second connection is a Universal Serial Bus (USB) connection that attaches to a car cigarette lighter 12volt adapter. Since all of USPS vehicles do not have a standard car cigarette lighter socket in the vehicle, an adapter cable was made to connect to the battery terminals in the vehicle. 


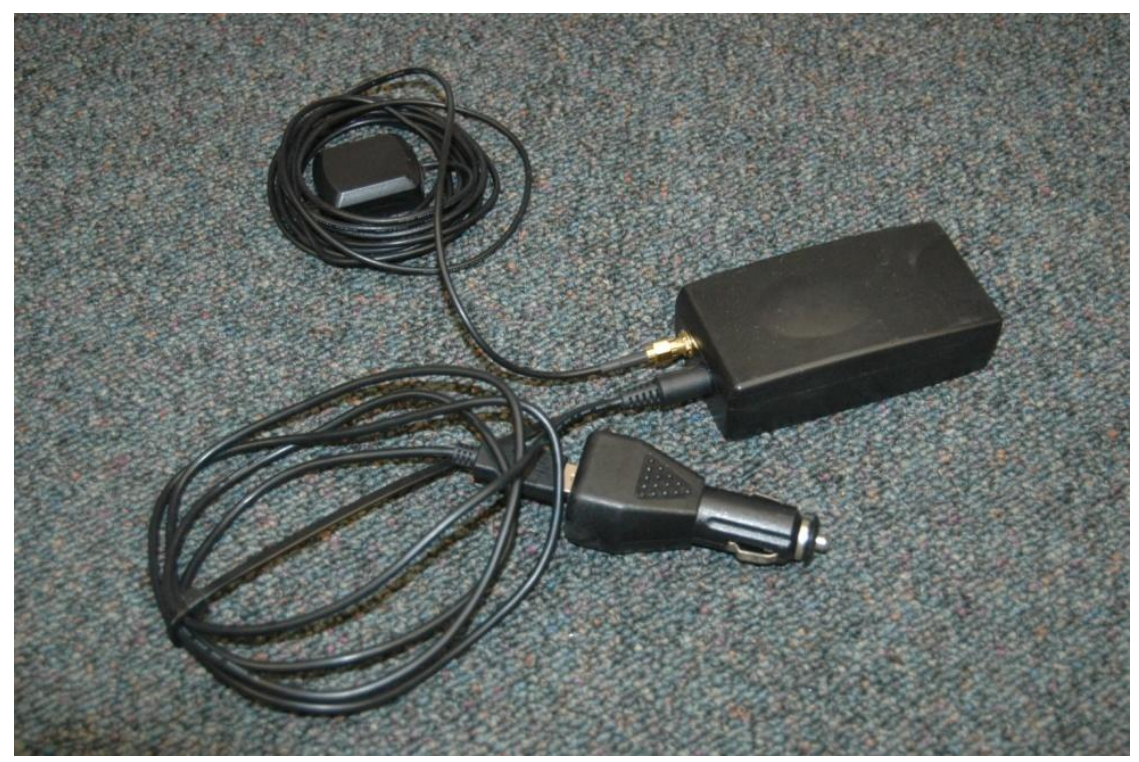

Figure 7. GPS unit used for the on road coastdowns

In order for a non-commercial driver to operate vehicle, its gross vehicle weight rating (GVWR) must be under the commercial vehicle limits of 26,001 lb. The GVWR for the vehicles were about 14,000 lbs, and the curb weights for the vehicles were about 10,000 lbs which allowed a non-commercial driver to operate this vehicle. Equation 1 below shows the calculation of the test weight for the vehicles where it requires the GVWR and curb weight. Both GVWR and the curb weight are shown in Table 20 and Table 21 in the appendixes.

$$
\text { Test Weight }=1 / 2 \times(G V W R-\text { Curb Weight })
$$

Equation 1. Test weight determination for the vehicles

In accordance to SAE J2711, the fuel economy results from a test cycle must be corrected for SOC after each run if they are to be considered valid. When a conventional vehicle completes a chassis cycle, the work done by the vehicle is equal to the work required to finish that cycle, and this is consistent from test to test. However, for a hybrid electric vehicle, which encompasses 
an energy storage system, there is a significant amount of energy stored in the vehicle which can be removed or added to the system during a cycle. In order to compare the results of a hybrid electric vehicle with the results of a conventional vehicle, the data must be corrected so that the energy change in the energy storage system is essentially zero [15]. The energy of the vehicle is the combination of the energy of the diesel fuel and at the battery storage system. SAE J2711 outlined the procedure for determining the energy comparison of the test cycle to energy change in the hybrid energy storage. Eaton Corporation provided WVU with Road Ranger software to monitor the battery storage system. The software was installed on a laptop and a Deutsch connector, in conjunction with Dearborn adapter, was used to monitor voltage and SOC of the energy storage system. The software did not provide any flexibility in monitoring the SOC and voltage during the test cycles. Hence, the state of charge and voltage could not be logged during the test cycle. It only provided instantaneous results. 


\section{Procedure}

\subsection{Empirical and Theoretical Model}

\subsubsection{Road Load Equation}

The purpose of the coastdown technique is to determine the rate of deceleration of a vehicle coasting. In order to determine the dynamometer settings from coastdowns, the first step is to evaluate the road load equation.

$$
P=\frac{1}{2} \rho C_{d} M A V^{2}+C_{r r} M g V+M V \frac{d V}{d t}+M g V \sin \theta
$$

Equation 2. Load road equation [7]

Where:

$\rho=$ density of air

$\mathrm{C}_{\mathrm{d}}=$ coefficient of drag

$\mathrm{M}=$ gravimetric mass of the vehicle

$\mathrm{A}=$ frontal area of the vehicle

$\mathrm{V}=$ velocity

$\mathrm{C}_{\mathrm{rr}}=$ coefficient of rolling resistance

$\mathrm{g}=$ acceleration due to gravity

$\sin \theta=$ road grade

$P=$ power

The first term in Equation 2 is the required power to overcome aerodynamic drag. In this term the density of air, frontal area of the vehicle, gravimetric mass of the vehicle, and velocity are all known or can be found using a known source. The $C_{d}$ is determined from the physical features of the vehicle from various types of testing, such as wind tunnel usage. For density of air, it was assumed to be $0.075 \mathrm{lbs} / \mathrm{ft}^{3}$, using a referenced standard temperature and pressure[34]. The gravimetric masses of the vehicles are given in Table 20 and Table 21 . The baseline vehicle and the hybrid vehicle are identical in shape and design, so for the frontal area, $\mathrm{A}, 71.25 \mathrm{ft}^{2}$ was 
used for both vehicles. This was determined by multiplying the maximum height of the vehicle by the maximum width of the vehicle.

The second term of Equation 2 is the required power to overcome rolling resistance of the tires. The gravimetric mass of the vehicle will be a combination of the test weight and the mass of the vehicle. As mentioned by Hucho, at a constant speed on a level road, rolling resistance usually exceeds aerodynamic drag at high speeds such as $50 \mathrm{mph}$ [34]. With the test schedule used for this project, a significant part of the test cycle consists primarily of acceleration and deceleration. The constant speed required to have $\mathrm{C}_{\mathrm{rr}}$ overcome $\mathrm{C}_{\mathrm{d}}$ in the test cycle is insignificant, because over $50 \%$ of energy from the fuel is used to accelerate the vehicle and over $30 \%$ is used for overcoming aerodynamic drag of the vehicle. Less than $10 \%$ of the energy is used to overcome $\mathrm{C}_{\mathrm{rr}}$ at high speeds.

The third term in the Equation 2 is the power required to overcome inertial force. This, as mentioned before, is simulated using large metal flywheels as shown in Figure 5.

The procedure for determining what configurations of flywheels are needed is provided in a technical paper by Wang et al. [29]. Table 2 below summarizes all the flywheel weight arrangements and the four weights that will be tested on the chassis dynamometer. For the hybrid vehicle, $1,770 \mathrm{lbs}$ were simulated through the flywheels and similarly for baseline vehicle 2,070 lbs were simulated. There were total of two empirical tests and two analytical tests.

Table 2. Test matrix of the weights and weight of the flywheels used

\begin{tabular}{|l|c|c|}
\hline & Hybrid Vehicle & Baseline Vehicle \\
\hline Curb Weight & $10,600 \mathrm{lbs}$ & $10,000 \mathrm{lbs}$ \\
\hline Test Weight & $1,770 \mathrm{lbs}$ & $2,070 \mathrm{lbs}$ \\
\hline Loaded Weight & $12,370 \mathrm{lbs}$ & $12,070 \mathrm{lbs}$ \\
\hline
\end{tabular}


The last term in Equation 2 is the power required for overcoming altitude change. This term was assumed to be zero during the evaluation of the coastdowns; this was because the variation of altitude change was minimal during the runs. Small variation was achieved by conducting the runs on the same strip of highway, but in the opposite direction. According to Cha, increasing the number of parameters to the model of the road load does not necessarily increase the accuracy of the results. The coefficients of the more complicated models derived by Cha became unstable during the regression analysis as the number of parameters increased. [35] A key note from Cha's study was the high rolling resistance achieved from his models. The higher rolling resistance maybe contributed from the losses in the drivetrain.

\subsubsection{Empirical Model}

The empirical model was developed from the on-road coastdowns conducted. The model involves determining the deceleration of the vehicle while no power is being delivered to the wheels through the transmission. A second order polynomial is the shape of the on road coastdown deceleration curve as shown by Equation 3 below.

$$
V=a_{3}+a_{2} t+a_{1} t^{2}
$$

Equation 3. Regression analysis of the deceleration curve

Where:

$\mathrm{V}=$ velocity

$\mathrm{t}=$ time

$a_{1}, a_{2}$, and $a_{3}=$ constants

In the equation above, constants $\mathrm{a}_{1}$ and $\mathrm{a}_{3}$ correspond to the rolling resistance of the vehicle and constant $\mathrm{a}_{2}$ correspond to aerodynamic drag of the vehicle. 
The PA controller requires a six digit input which would represent the road loads.

The constants can be evaluated using numerical analysis by applying regression analysis to the speed versus deceleration model, the shape of this curve would be a third order polynomial as shown in Equation 4 below.

$$
D=b_{4}+b_{3} v+b_{2} v^{2}+b_{1} v^{3}
$$

Equation 4. Regression analysis of deceleration versus speed

Where:

$\mathrm{D}=$ deceleration

$\mathrm{V}=$ velocity

$\mathrm{b}_{1}, \mathrm{~b}_{2}, \mathrm{~b}_{3}$, and $\mathrm{b}_{4}=$ constants

\subsubsection{Theoretical Model}

For the Theoretical model setup of the vehicle, THDVETL followed the procedure set by the 40 CFR part 86 subpart M section 1200 which had a large involvement in the chassis dynamometer setup [36]. Even though 40 CFR part 86 subpart M section 1200 was test for evaporative emissions, the procedure it outlines for chassis dynamometer calibration, pretests, and determination of road load power can be used for all types of tests. To determine the two coefficients of $\mathrm{C}_{\mathrm{d}}$ and $\mathrm{C}_{\mathrm{rr}}$ the Equation 5 below was applied: 


$$
R L P=F \times 0.67 \times(H-0.75) \times W+0.00125 \times L V W
$$

Equation 5. Road load equation as provided by the 40 CFR part 86 subpart M [36]

Where:

RLP $=$ Road Load Power in Horsepower at $50 \mathrm{mph}$

$\mathrm{F}=1.00$ for tractor trailers, 0.85 for urban buses

$\mathrm{H}=$ Maximum Height of the vehicle in feet

$\mathrm{W}=$ Maximum Width of the vehicle in feet

LVW $=$ Loaded Vehicle Weight in Pounds

If aerodynamics are evaluated as function of height and width; and rolling resistance as function of weight primarily, then Equation 5 can be split up in to two terms. In the first term, height and width are known variables, so this can be equal to the first term of Equation 2. Similarly, the second term of Equation 5 can be equaled to the second term of Equation 2. All the variables in the two equations are known except for $\mathrm{C}_{\mathrm{d}}$ and $\mathrm{C}_{\mathrm{rr}}$ which can be solved for using speed of $50 \mathrm{mph}$ as a reference speed.

\subsection{Vehicle Preparations and Coastdowns Procedure}

The coastdowns and the FTP test were done in July and August of 2008. When the vehicles arrived at West Virginia University, they were inspected for proper tire pressure and the fuel tank was filled before determining the weight of the vehicle. An A. P. T. Axle Weigher produced by Intercomp was used to determine the weights of the vehicles. The hybrid vehicle weighed about 600 pounds more than the baseline vehicle due to extra components. These included the battery storage, control box, electric motor, and any other extra parts from being hybridized. The weights of the hybrid vehicle and baseline vehicle were approximately 10,600 and 10,000 pounds, respectively. 
West Virginia's terrain was too mountainous and the variation in the elevation would have voided the coastdown tests. An assumption that was made during the road load determination was that the coastdown was done on a flat road. Therefore, the coastdowns were done on PA-43 in Pennsylvania which is about 45 minutes north of Morgantown, West Virginia. The PA-43 is relative flat terrain and the low traffic makes a suitable place for coastdowns. A map of the road, which spans about 10 miles, is shown Figure 8.

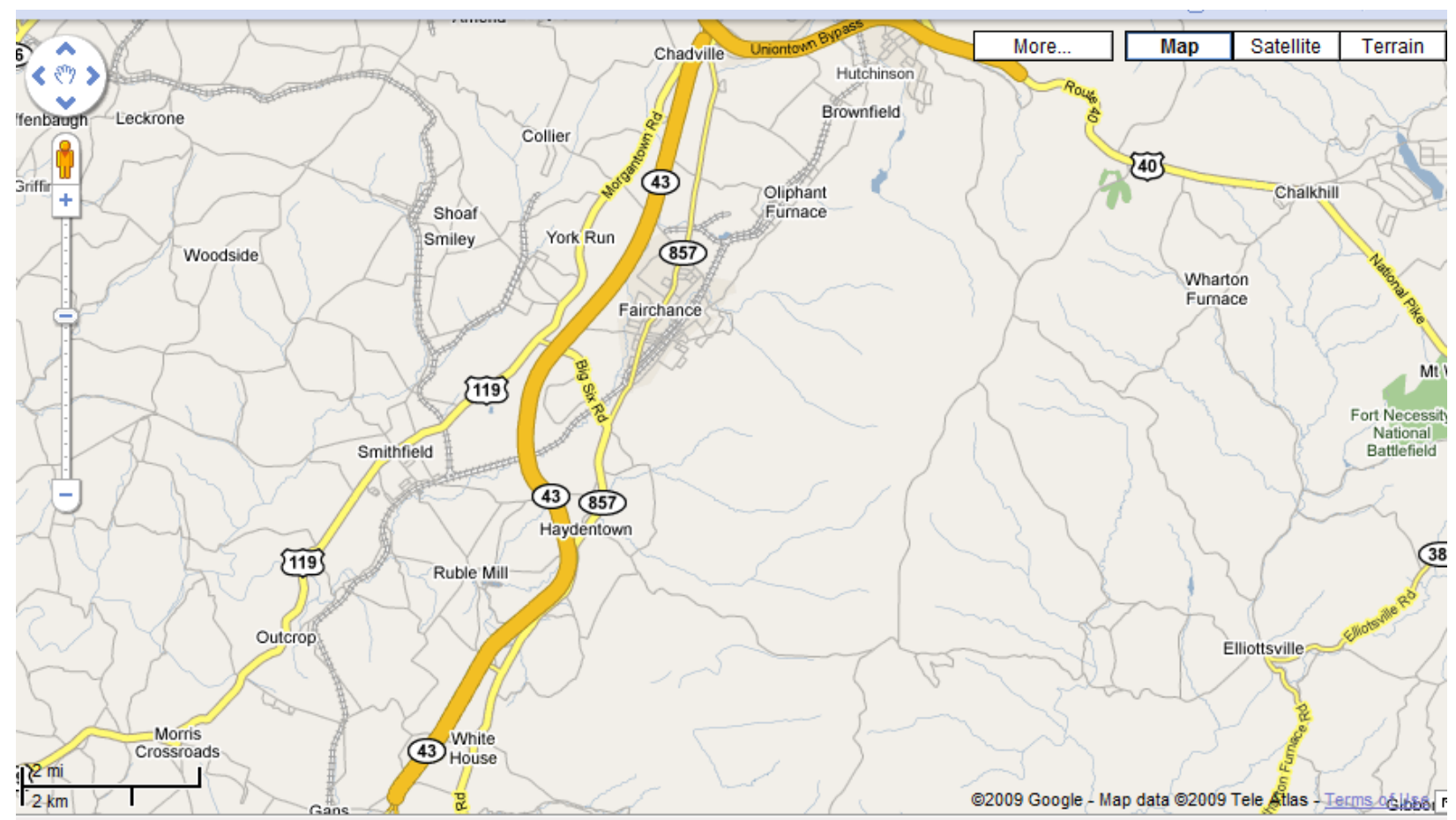

Figure 8. PA-43, which stretches about 10 miles, is where the coastdown tests were conducted [30]

Once the vehicle arrived to its destination on PA-43, the GPS was activated. A stretch of straight road and a minimal elevation change was where the coastdown procedure began. Even though the data could be extracted from the coastdowns on mountainous road, the error would have arisen from the data reduction. The vehicle was accelerated until it reached $50 \mathrm{mph}$ than the 
transmission was set to neutral. At this point there was no power going to the wheels through the transmission and vehicle began coasting until it reached zero mph. A similar procedure was attempted again except on the opposite side of the road going the opposite way. The purpose of this was to avoid any change in elevation when the averages of the coastdowns were evaluated for road load characterization. Multiple runs were attempted and the average time for the coasting period was a little over two minutes. Figure 9 below illustrates the altitude profile of the exact place where the coastdowns were conducted on PA-43. The GPS unit provides satellite location and using Google, altitude profile can be drawn for that location.

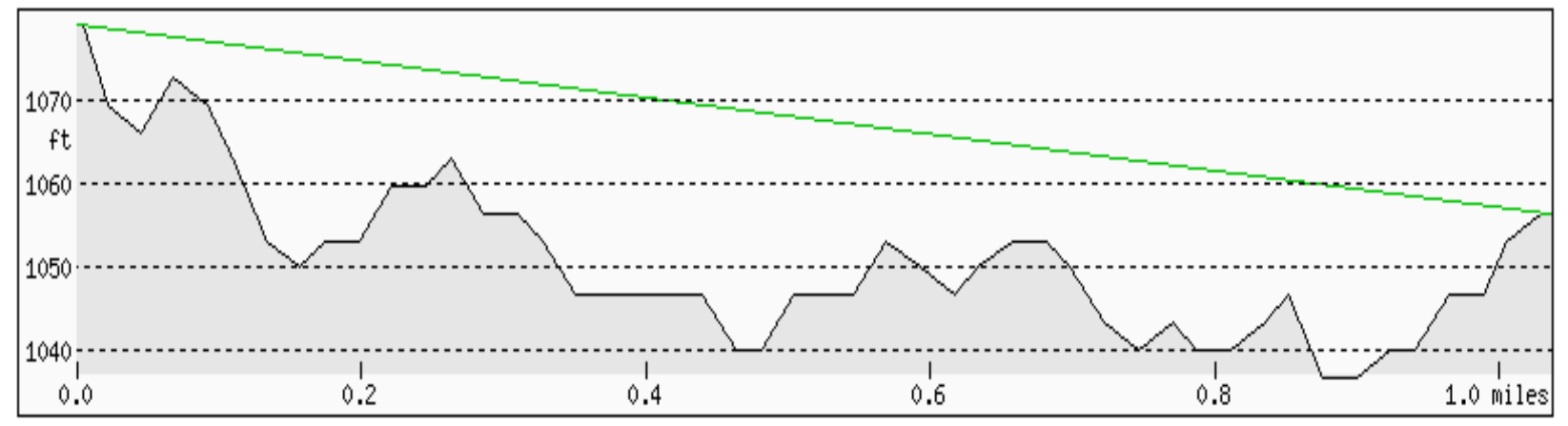

Figure 9. Elevation profile of the road where the coastdowns were conducted [31]

For the baseline vehicle, coastdowns were done with the loaded configuration and the unladen configuration. Calibrated weights from WVU's engine laboratory were used as a cargo load. The weights were configured in $25 \mathrm{lbs}, 50 \mathrm{lbs}$, and $10 \mathrm{lbs}$. The unladen configuration was the vehicle's weight only for the coastdown. Due to the time constraint of the project, the hybrid vehicle coastdown was only done with the unladen configuration. Equation 1 was used to determine the test weight of the vehicles during general testing. Due to changes in weather conditions between test days it was difficult to see any change from the cargo loads. 


\subsubsection{Vehicle Installation and Instrumentation}

The vehicles were driven onto the chassis dynamometer rollers where they were balanced between two rollers. The front wheels of the vehicles were raised using heavy duty jacks, placed on stands, and was leveled with the back wheels as illustrated by Figure 3. The rear axles housing of the vehicles were chained to the dynamometer beds to avoid losing friction between the tires and the rollers. The tensions in the chains are not as tight as possible due the over flexing of the tires on the rollers. Study done by Clark et al. [37] concluded that the deflection of the tire on the flat service was smaller than that on drum roll. The excesses tension on the chains could result in the vehicle in doing more work to overcome the stresses in tires due to deflection. For the hybrid vehicle, a Dearborn 4 adapter was connected the ECU in order to monitor SOC and nominal voltage during the FTP-75 test cycles. The driver was provided with a visual trace of the scheduled speed versus time on a video monitor. The video monitor displayed the prescribed speed while the actual speed was displayed as a line trace.

\subsubsection{Vehicle Coastdown on Chassis Dynamometer and Determination of Parasitic Losses}

Before conducting the test scheduled for each of the vehicles, THDVETL was required to do a few preliminary procedures according to test protocols developed at WVU. The vehicle must be operated on the dynamometer for minimum of ten minutes to increase the temperatures of the tires, and the fluids in the axles as well as transmissions. If the tires' temperatures are relatively low, the vehicle tends to vibrate excessively during the test drives due to the stiff 
rubber. The temperature of the oil is warmed up to $100^{\circ} \mathrm{F}$ to decrease viscosity and subsequently reduce losses in the system.

As discussed in previous section, the $25 \mathrm{HP}$ AC motor is used to overcome system losses during the test. These parasitic losses include friction from all the bearings, differentials, and universal joint couplings on the medium duty chassis dynamometer. An SAE technical paper by Wang et al. discusses the determination of the parasitic losses for a chassis dynamometer [38]. This paper develops a theoretical model for energy consumption which describes the mechanics of the vehicle system, the motion experience of the system, and predicts the capabilities of the system performance.

There are two methods of determining the parasitic losses for a dynamometer. The first is the steady-state method, where the vehicle is driven at various constant speeds and the corresponding torques are measured at the driving wheels. From the vehicle system equation, a coefficient of the losses can be determined from least square approximation. The other method was coastdown, which is the selected method for this report. The vehicle is driven to a specific speed, at which the transmission is set to neutral, and the vehicle is allowed to decelerate freely. A velocity time characteristic is obtained from which the coefficients of the losses are determined using least square curve. Equation 6 below shows the equation derived for parasitic losses of the chassis dynamometer. [38]

$$
T=a_{1}+a_{2} \omega^{2}
$$

Equation 6. Parasitic losses of the chassis dynamometer

Where:

$\omega=$ speed of the rollers

$\mathrm{a}_{1}$ and $\mathrm{a}_{2}=$ constants

$\mathrm{T}$ - torque 


\subsubsection{FTP -75 Test Procedure and State of Charge Determination}

Before the actual FTP-75 was conducted a few issues arose. The vehicle's ECU showed a faulty code when it recognized the vehicle's front wheels were not moving through the Anti-lock Brake System (ABS) unit. This was corrected by using the signal from the back wheels ABS unit and rerouting back to the front; initially setting up a parallel system. Once the vehicle was prepared and warmed up, the driver began the FTP-75 test cycle by following a trace provided by the data acquisition system from the trailer. The driver followed the trace as close as possible during the 30 minute period of the test.

For the hybrid vehicle, the SOC was monitored during the FTP-75 test cycle in order to ensure correct energy consumption of the vehicle in accordance with J2711. For conventional internal combustion engine systems, the energy source (fuel) used by the vehicle to complete the test would be very consistent from run-to-run. For hybrid drive systems, fuel energy may be stored, and stored energy may be depleted during a given test run. Therefore, an energy correction must be performed so that vehicle performance characteristics are normalized by energy required to complete the test cycle. SOC correction will help to reduce test-to-test variation, and provide a more representative energy characterization by which performance may be normalized.

To determine if a test run has an acceptable NEC, that does not require SOC correction, divide NEC by total cycle energy. If the absolute value of the calculation yields a percentage less than or equal to $1 \%$, the NEC variance is within tolerance. The emission and fuel economy values for the test run do not need to be corrected for SOC.

If the absolute value of the calculation yields a percentage greater than $1 \%$, but less than or equal to $5 \%$, emission and fuel economy values from the test run need to be corrected for SOC 
as described below. Test runs with NEC variance greater than $\pm 5 \%$ are considered invalid or, if the vehicle is consistently depleting charge, may have to be tested under the charge-depleting vehicle recommendations.

In order to compute a state of charge correction for fuel economy, the fuel economy values for each run must be plotted against the NEC for each run. A linear interpolation (in some cases extrapolation may be allowed) is performed to establish the fuel economy at a NEC of zero (i.e., the data is corrected to reflect a net zero change in SOC). The equation below was used to determine the NEC of Eaton's energy storage system.

$$
N E C=\left(S O C_{\text {final }}-S O C_{\text {initial }}\right) \times V_{\text {system }} \times K_{1}
$$

Equation 7. Net energy change in batteries [15]

where:

$\mathrm{SOC}_{\text {initial }}=$ Battery SOC at the beginning of the test cycle

$\mathrm{SOC}_{\text {final }}=$ Battery SOC at the end of the test cycle

$\mathrm{V}_{\text {system }}=$ Nominal Voltage of the battery

$\mathrm{K}_{1}=$ Conversion factor

Equation 8 below was used to determine the total fuel energy consumed by the vehicle. The total fuel energy will be compared to the NEC of the batteries in order to determine the \% change.

$$
\text { Total Fuel Energy }=N H V_{\text {fuel }} \times m_{\text {fuel }}
$$

Equation 8. Fuel Energy consumed by the vehicle [15]

where:

$\mathrm{NHV}_{\text {fuel }}=$ Net heating value per consumable fuel analysis as specified by ASTM D240 or D 4809 $\mathrm{m}_{\text {fuel }}=$ Total mass of the fuel consumed over the test cycle 


\section{Results}

The results are representative of the coastdowns conducted on PA-43, coastdowns conducted on the chassis lab, and the all of the FTP-75 tests. Individual test results for each the different methods of coastdown are provided in the appendixes as well as individual on road coastdown evaluation.

\subsection{Coastdown}

\subsubsection{On-road Coastdowns}

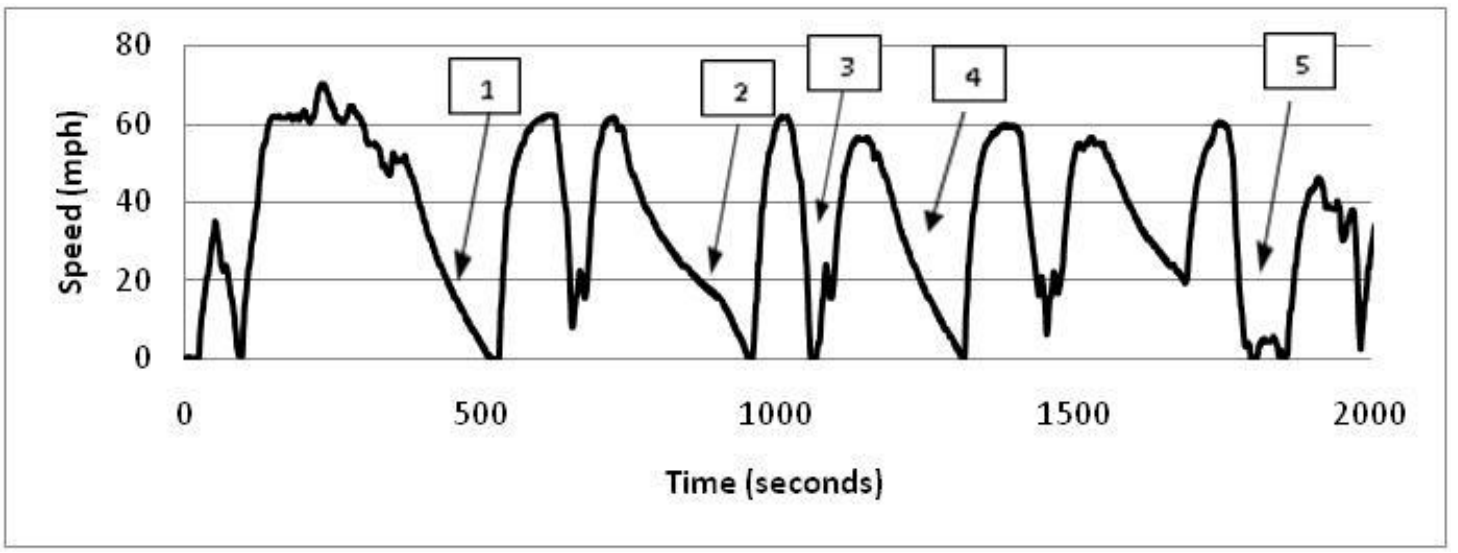

Figure 10. A set of data from the GPS unit during the hybrid vehicle Coastdown on PA-43

Figure 10 above shows a set of results from the hybrid vehicle on-road coastdowns in which test runs 1 and 4 are two coastdowns that were considered valid. Starting from around 50 $\mathrm{mph}$, the speed of the vehicle steadily decreased to $0 \mathrm{mph}$ and took a period of about 140 seconds. Coastdown 2 was another run, but starting from $20 \mathrm{mph}$ an increase of speed occurred causing it to be invalid. The sudden change in speed for run 2 could be the result of sudden 
increase in wind, significant change in altitude, or lane change due to safety precaution. Sections 3 and 5 represent the vehicle braking while on an on-ramp and an off-ramp.

A closer investigation of the runs in Figure 30 and Figure 31 in the appendixes shows how run 2 was considered invalid. From the elevation change in Figure 31, it shows that the vehicle began coasting at a lower altitude then it increased too much higher altitude and decreased once again. The increase in the elevation during test run 2 led to the increase in the period of the coastdown of about 170 seconds.

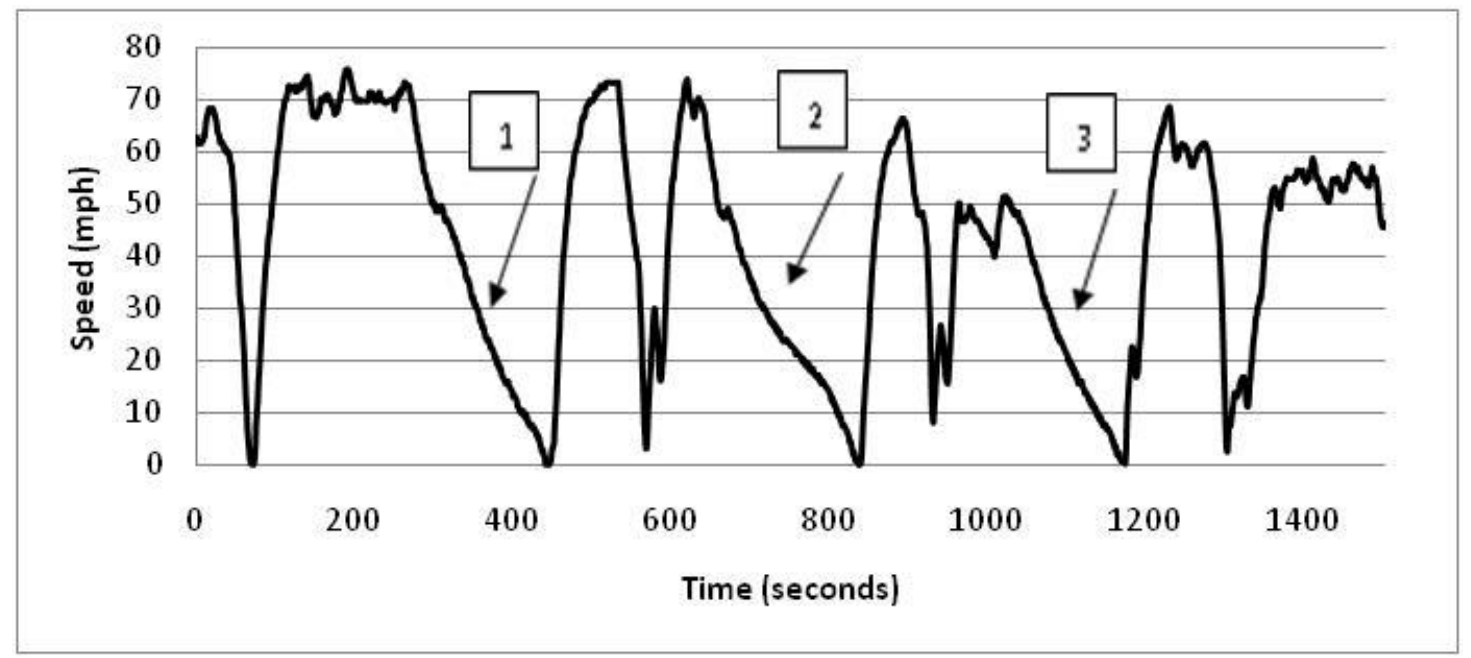

Figure 11. Set of data from the GPS during the unladen baseline vehicle coastdown 


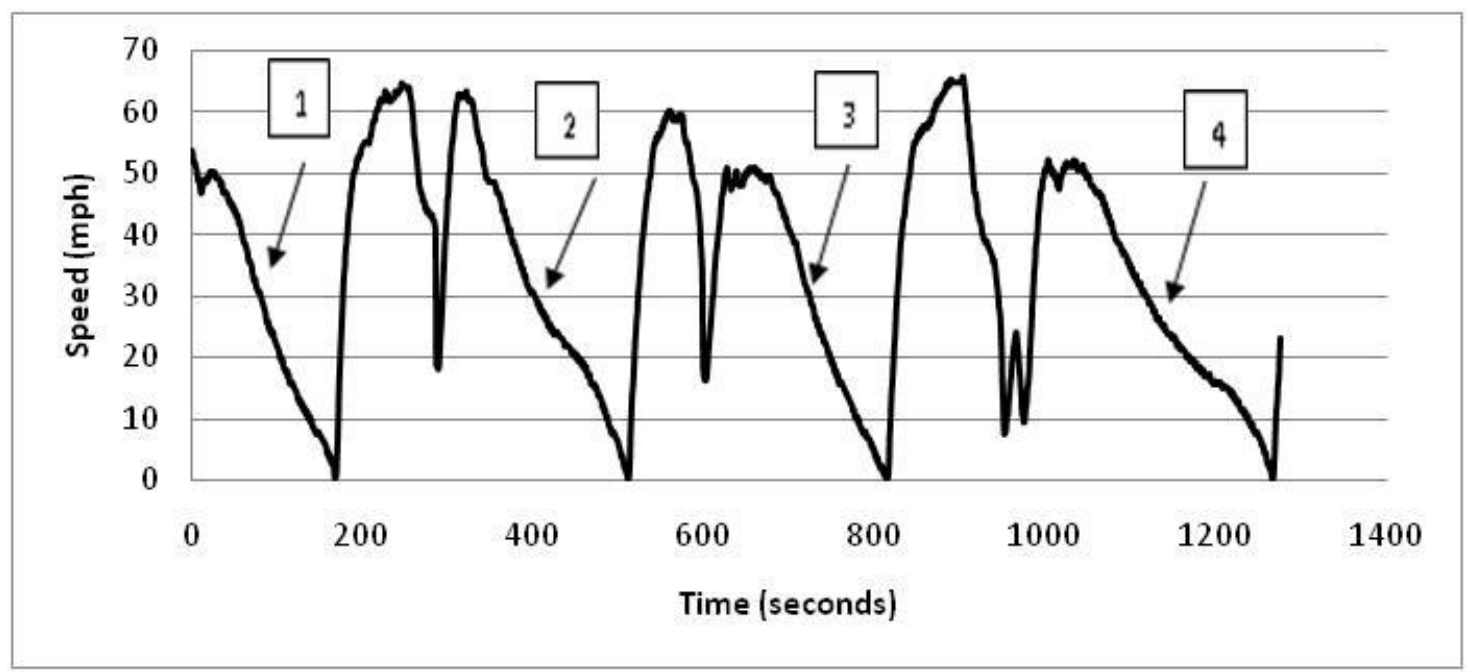

Figure 12. Set of data from the GPS during the loaded baseline vehicle emission

Figure 11 and Figure 12 show the GPS data for the unladen baseline vehicle and loaded baseline vehicle, respectively. For the unladen baseline vehicle, the GPS data provided only two valid runs. Two valid runs were also evaluated from the loaded baseline vehicle. In Figure 11, 
runs 1 and 3 were chosen to be evaluated and the results of the runs can be seen in Figure 32 and

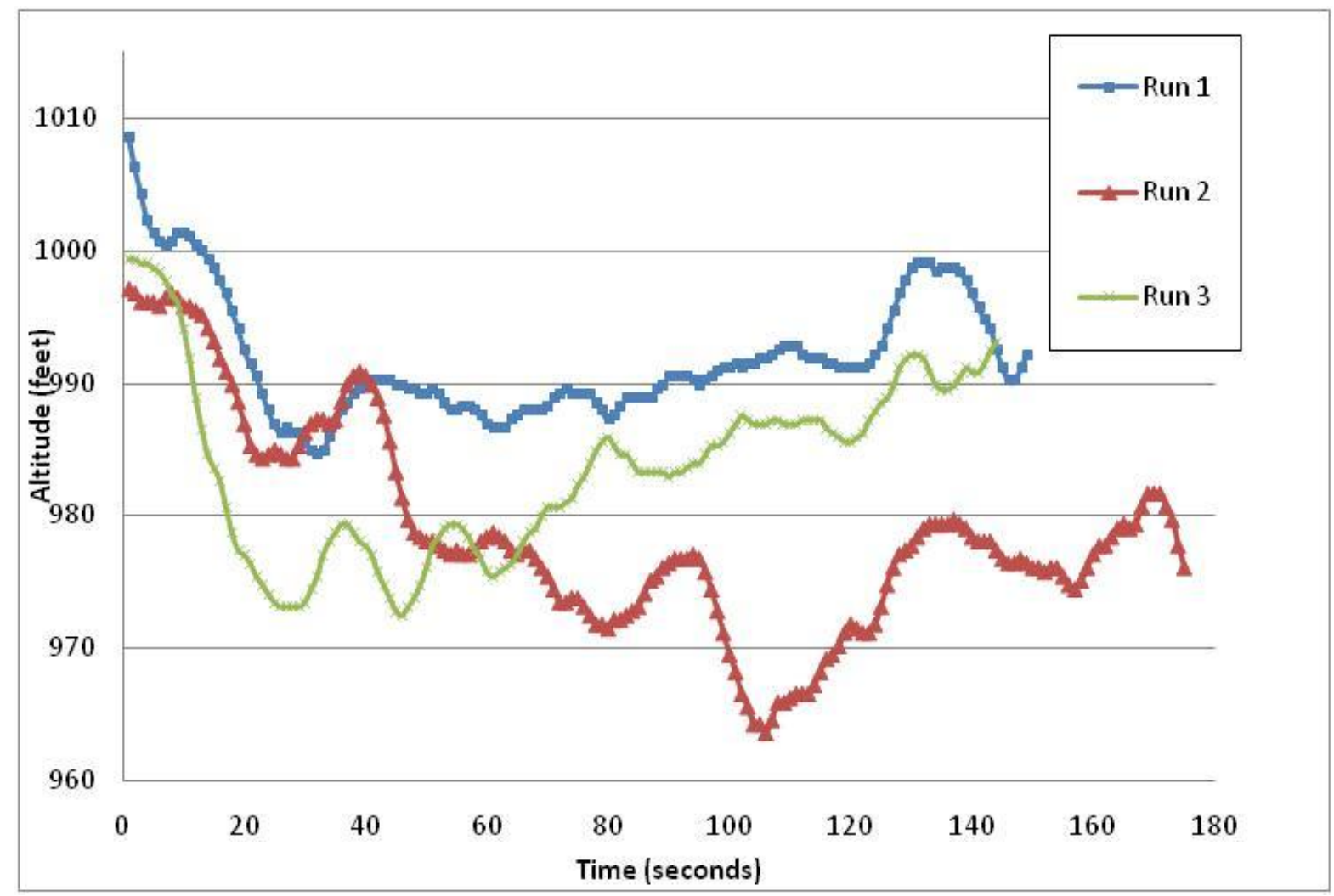

Figure 33 in the appendixes. Out of four runs from the loaded baseline vehicle only two were used, runs 1 and 3. Figure 34 and Figure 35 in the appendixes shows how change in elevation drastically causes an increase speed and longer coastdown period.

Figure 13 shows the statistical comparison of the average coastdowns for all three vehicles. The maximum standard deviation is about 2.64 , but the coefficient of variation is about $58 \%$. Looking closer at the Figure 13 where the COV is much higher compared to the rest of the results, it may be inferred that rolling resistance of the different tires and conditions of the tires may contribute to the variation in the results. A small change in pressure of the tire causes significant change in the rolling resistance of the vehicle [23]. Also, COV towards the end of the runs increases due to low speed and a significant change in $\mathrm{COV}$ was seen due to small changes in speed. 


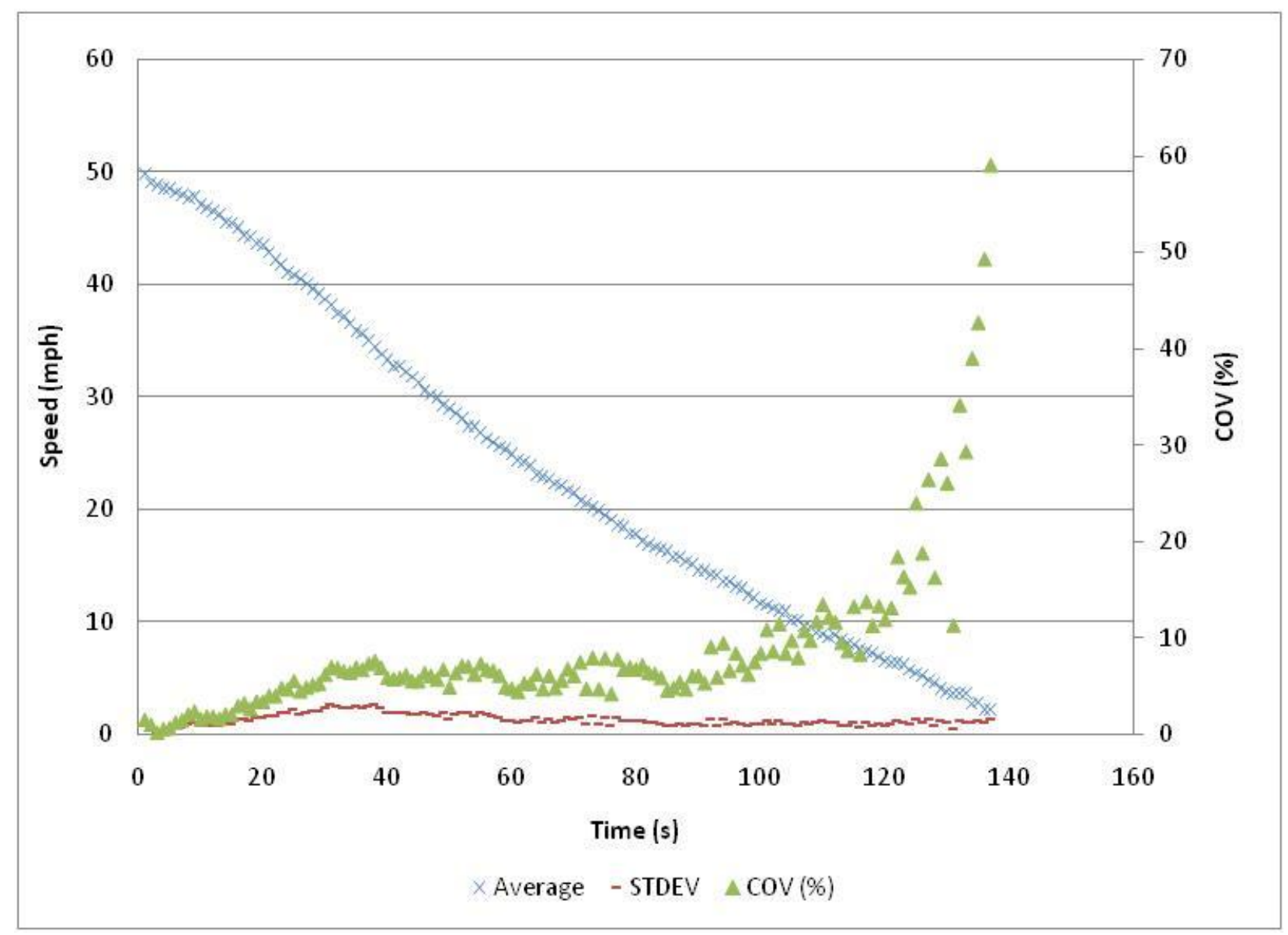

Figure 13. The statistical information about the average coastdowns of both vehicles

Figure 14 shows the statistical comparison of the baseline vehicle for both loaded and unladen on-road coastdown methods. At higher speeds, the aerodynamic influence, which is the $3^{\text {rd }}$ term of the regression equation, is apparent. The curviness of the plot represents the aerodynamic drag and shows that the force exerted on the vehicle is primary from that. Rolling resistance is also a significant contributor to the force exerted and should be ignored; this can be more noticeable at lower speeds [23]. 


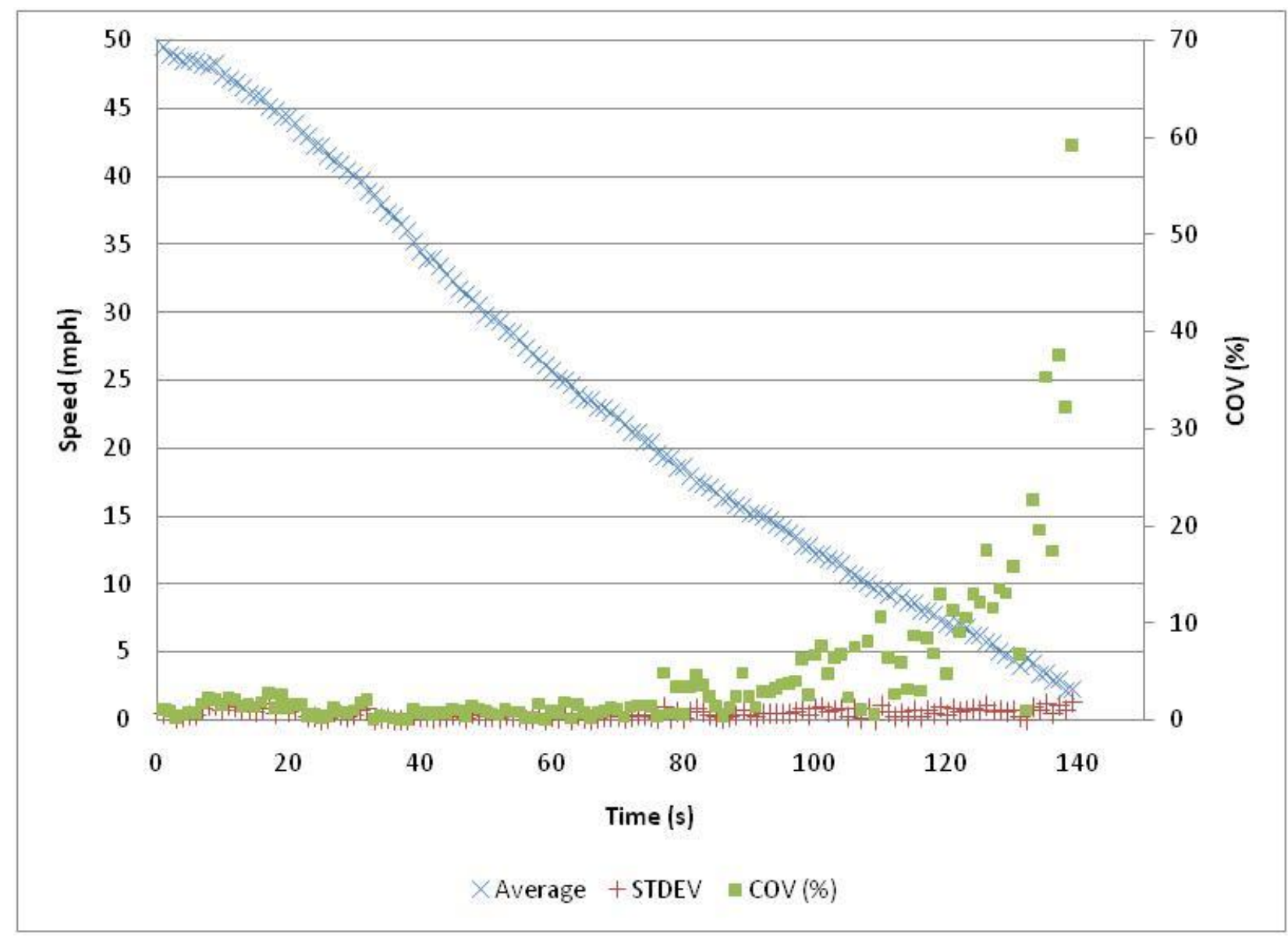

Figure 14. The comparison the loaded coastdown and unladen coastdown using the baseline vehicle 


\subsubsection{Evaluation of Coastdowns}

Figure 15 shows the results of the on road coastdowns conducted on PA-43. The red and the blue lines are from the baseline vehicle with relatively similar characteristics as can be seen by the regression analysis. This can be explained by a windy condition, where aerodynamic drag is a function of speed squared having a nonlinear characteristic. The loaded vehicle took longer to coast to a vehicle speed of zero as expected and as can be seen in the figure below.

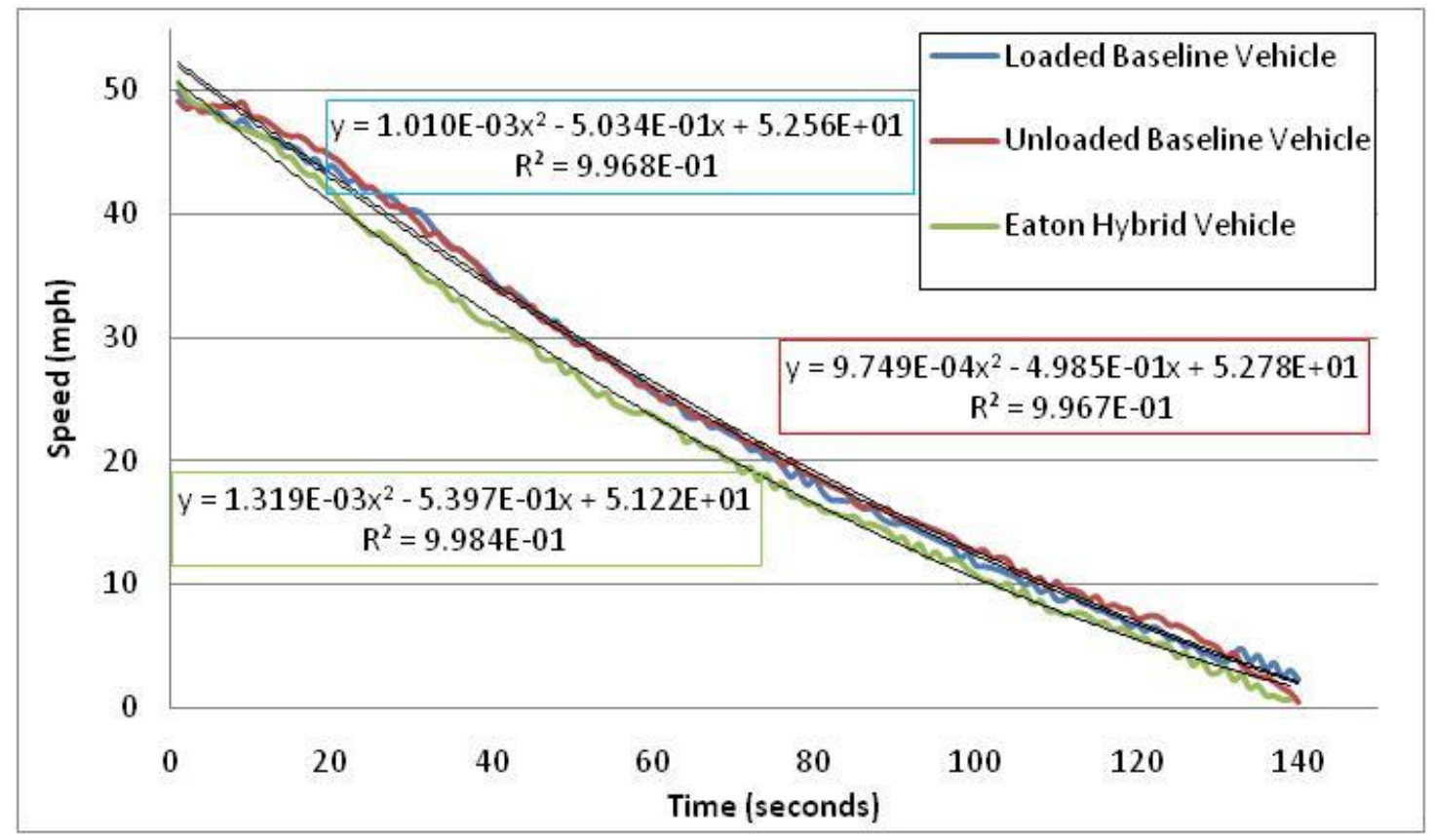

Figure 15. The comparison of the average coastdowns with regression analysis 
Figure 16 shows the deceleration of all vehicles versus speed with blue and red lines representing the baseline vehicle and the green line representing the hybrid vehicle. It should be noted that all the coastdowns were conducted on different days. The coastdowns of the baseline vehicle were conducted on the same week which had similar weather condition. The hybrid vehicle seems to have a more aggressive deceleration at the higher speed and smoother deceleration at lower speeds.

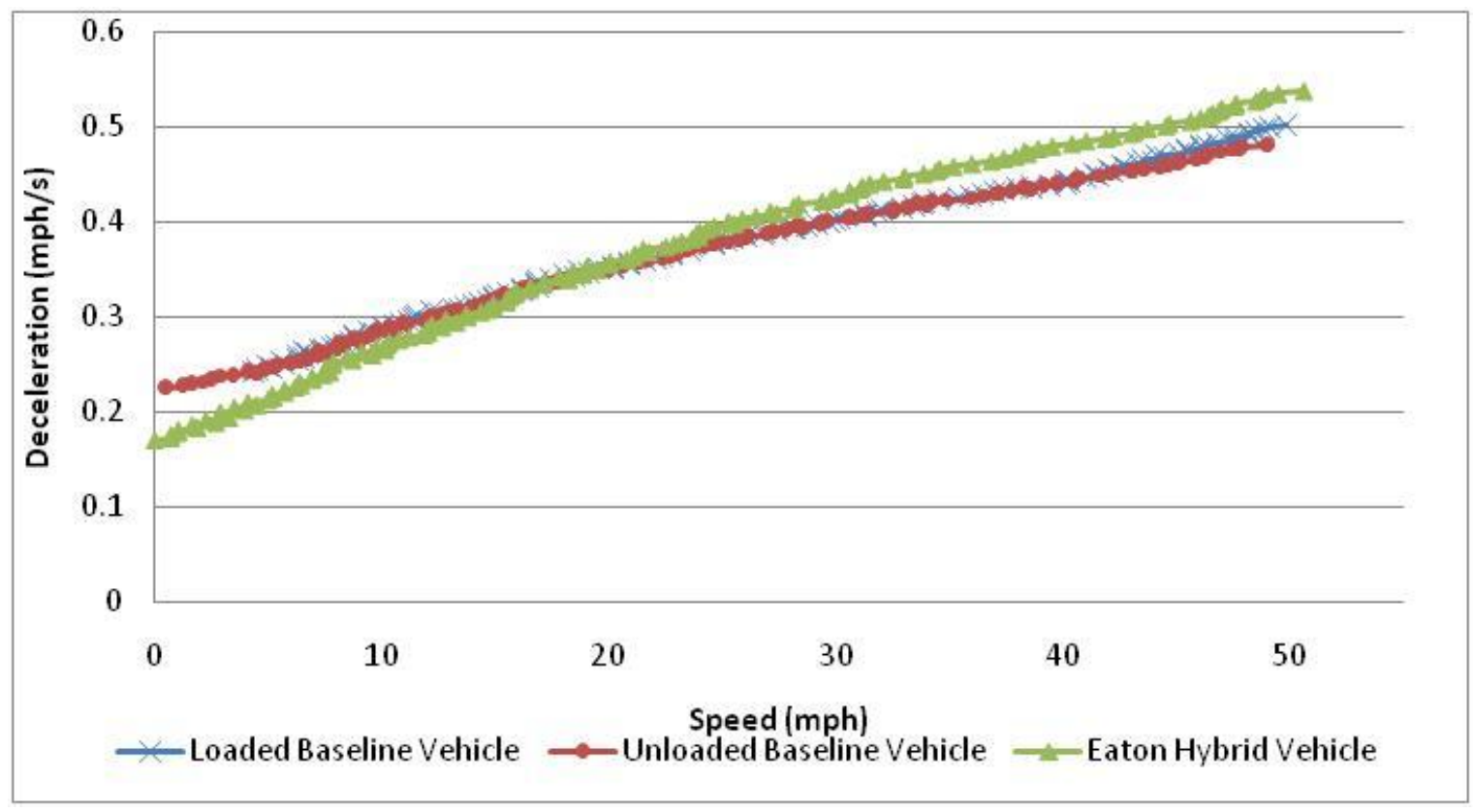

Figure 16. The deceleration of all the vehicles with respect to speed 
Figure 17 shows how similar the baseline vehicles' coastdowns are. The loaded vehicle has a little higher influence than unladen vehicle as expected. The added weight for the loaded vehicle only affects the rolling resistance term.

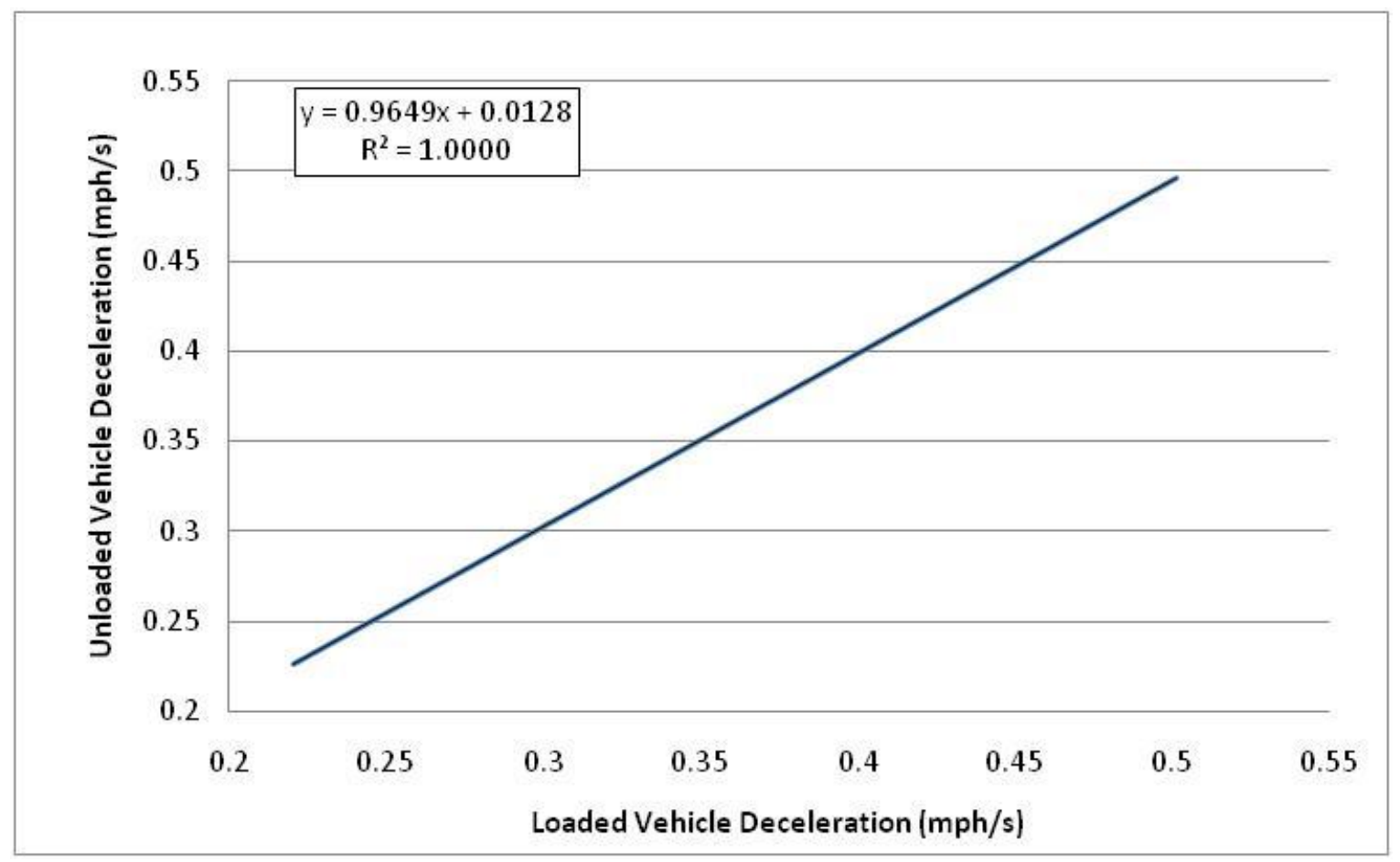

Figure 17. The regression analysis of the loaded versus the unladen baseline vehicle coastdowns. 


\subsubsection{Empirical and Theoretical Model Results}

The empirical model will mainly be based on the coastdown done with the hybrid vehicle and baseline vehicle on the PA-43 road. Coastdowns from the GPS provided speed versus time data and from this an average of runs were taken, as shown in Figure 30, Figure 32, Figure 34 and Figure 15. In Table 3, the effect of the test weight between the two baseline vehicles can be seen. The third term in Table 3 below represents the influence of the $C_{d}$ which consists of the vehicle's external design and wind turbulence. The first and the second term represent the $\mathrm{C}_{\mathrm{rr}}$ of the vehicle. The added test weight would not have an effect on the $\mathrm{C}_{\mathrm{d}}$ due to the fact the weights were placed inside of the vehicle and had no way of increasing the frontal area. The $3.5 \%$ change in the third term can contributed from the variation in wind direction during the runs. The coastdowns for the baseline vehicles were conducted on separate days of the week. The $\mathrm{C}_{\mathrm{rr}}$ would have been affected more with the increase of the vehicle weight then $\mathrm{C}_{\mathrm{d}}$, but coastdowns only showed a change of about one $\%[20,32]$.

Table 3. The characteristics of the coastdown curves

\begin{tabular}{|l|c|c|c|c|}
\hline \multicolumn{5}{|c|}{ Average Coastdown Characteristics } \\
\hline Vehicle & 3rd Term & 2nd Term & 1st term & R squared \\
\hline Eaton Hybrid & $1.319 \mathrm{E}-03$ & -0.5397 & 51.22 & 0.9984 \\
\hline Unladen Baseline & $9.749 \mathrm{E}-04$ & -0.4985 & 52.78 & 0.9967 \\
\hline Loaded Baseline & $1.010 \mathrm{E}-03$ & -0.5034 & 52.56 & 0.9968 \\
\hline
\end{tabular}

A linear regression was applied to average coastdowns to determine the deceleration of the vehicles. From Equation 2, if it is assumed the changes in road grade is minimal and no power is delivered to the wheels, it can be written as: 


$$
\frac{d v}{d t}=\frac{\rho A C_{d}}{2 m} v^{2}+g C_{r r}
$$

Equation 9. Deceleration of the vehicle

In Equation 9, $\frac{\rho A C_{d}}{2 m}$ and $g C_{r r}$ are constants and by least-square-error regression of acceleration $\left(\frac{d v}{d t}\right)$ versus velocity squared can be used to determine the unknown coefficients. The acceleration $\left(\frac{d v}{d t}\right)$ can be derived from the coastdown results. Density of air, frontal area, weight, and gravitational force are all known values in this equation [33[. From Equation 9, $\mathrm{C}_{\mathrm{d}}$ equated to 0.669 and the $\mathrm{C}_{\mathrm{rr}}$ equated to 0.0139 . The $\mathrm{C}_{\mathrm{d}}$ coefficient for these vehicles from the empirical formula was a reasonable value based on information for commercial vehicles, but the $\mathrm{C}_{\mathrm{rr}}$ coefficient was a little high [34]. The road load equation does not account for the losses in the drivertrain, hence it equated in $\mathrm{C}_{\mathrm{rr}}$.

For the Theoretical model, using Equation 5 and an F of 1 as assumed, the road load power at $50 \mathrm{mph}$ can be estimated. The other variables in Equation 5 are known. The following results were provided from the Theoretical model shown in Table 4.

Table 4. Road load power required at $50 \mathrm{mph}$

\begin{tabular}{|l|r|r|}
\hline RLP (HP) & \multicolumn{1}{|c|}{ Loaded } & \multicolumn{1}{c|}{ Unladen } \\
\hline Baseline Vehicle & 58.81 & 56.22 \\
\hline Hybrid Vehicle & 59.18 & 56.97 \\
\hline
\end{tabular}

Using the derived Equation 9 and the results from

Table 4, the coefficients $C_{d}$ and $C_{r r}$ was evaluated and provided in the Table 5. The frontal area of both the hybrid vehicle and baseline vehicle are identical, hence the nearly similar $C_{d}$ coefficient. 
Unless the weight of the vehicle is significantly different by a magnitude of $10,000 \mathrm{lbs}, \mathrm{C}_{\mathrm{rr}}$ is constant.

Table 5. The results of the Theoretical $\mathrm{C}_{\mathrm{d}}$ and $\mathrm{C}_{\mathrm{rr}}$ based on the EPA model

\begin{tabular}{|l|c|c|}
\hline & $\mathbf{C}_{\mathbf{d}}$ & $\mathbf{C}_{\mathbf{r r}}$ \\
\hline Baseline Vehicle & 0.735 & 0.00937 \\
\hline Hybrid Vehicle & 0.735 & 0.00937 \\
\hline
\end{tabular}

\subsubsection{Evaluation of Chassis Coastdowns Results}

Figure 18 and Figure 19 show the results of the chassis dynamometer coastdown runs. Both figures show distinct differences between the theoretical and empirical coastdowns. Figure 20 below shows all the coastdowns conducted for this study. The on-road coastdowns are the average of multiple runs conducted on PA-43 where the dynamometer coastdown consists of one run for each method. 


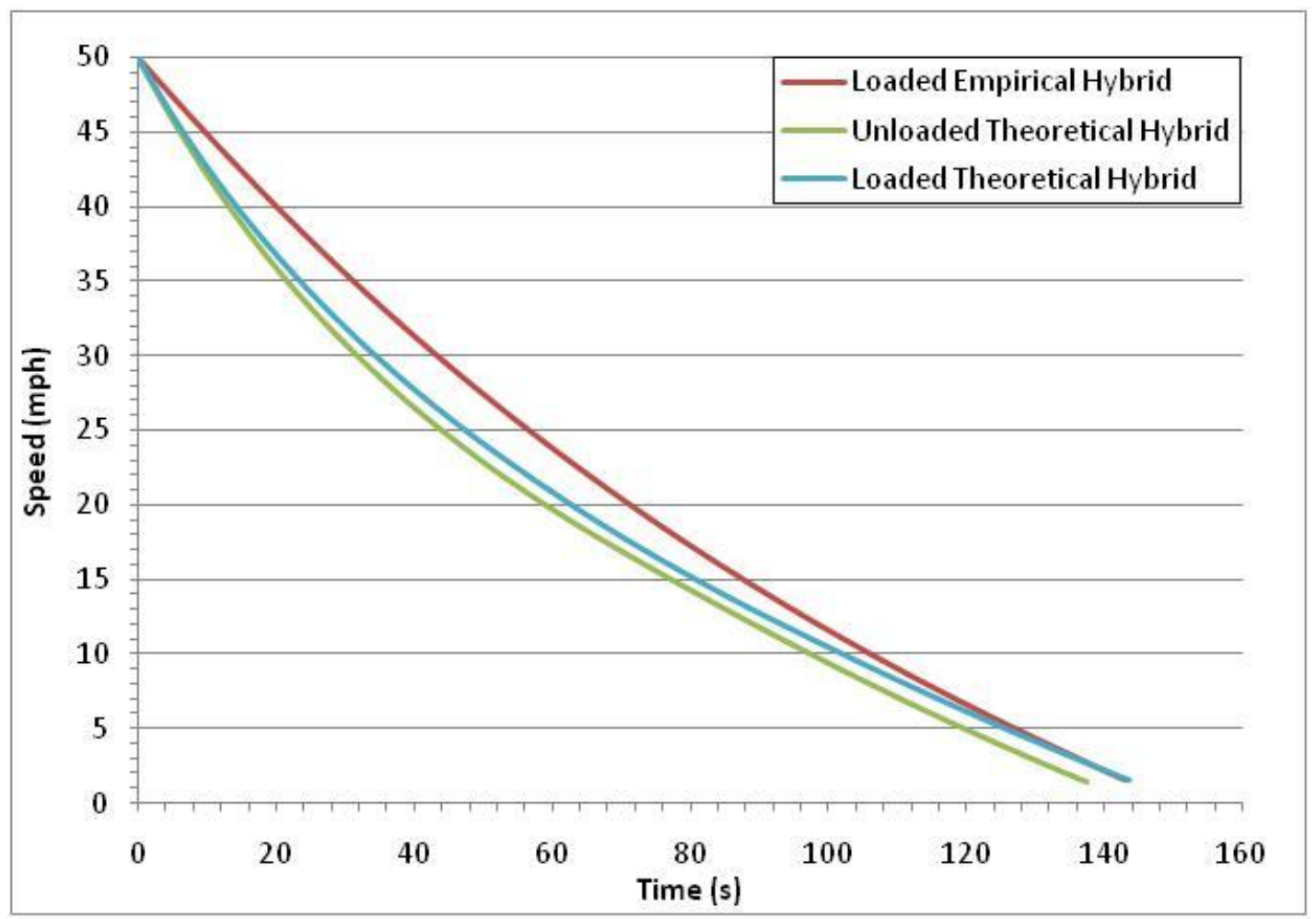

Figure 18. Comparison of the chassis coastdown runs for the hybrid vehicle

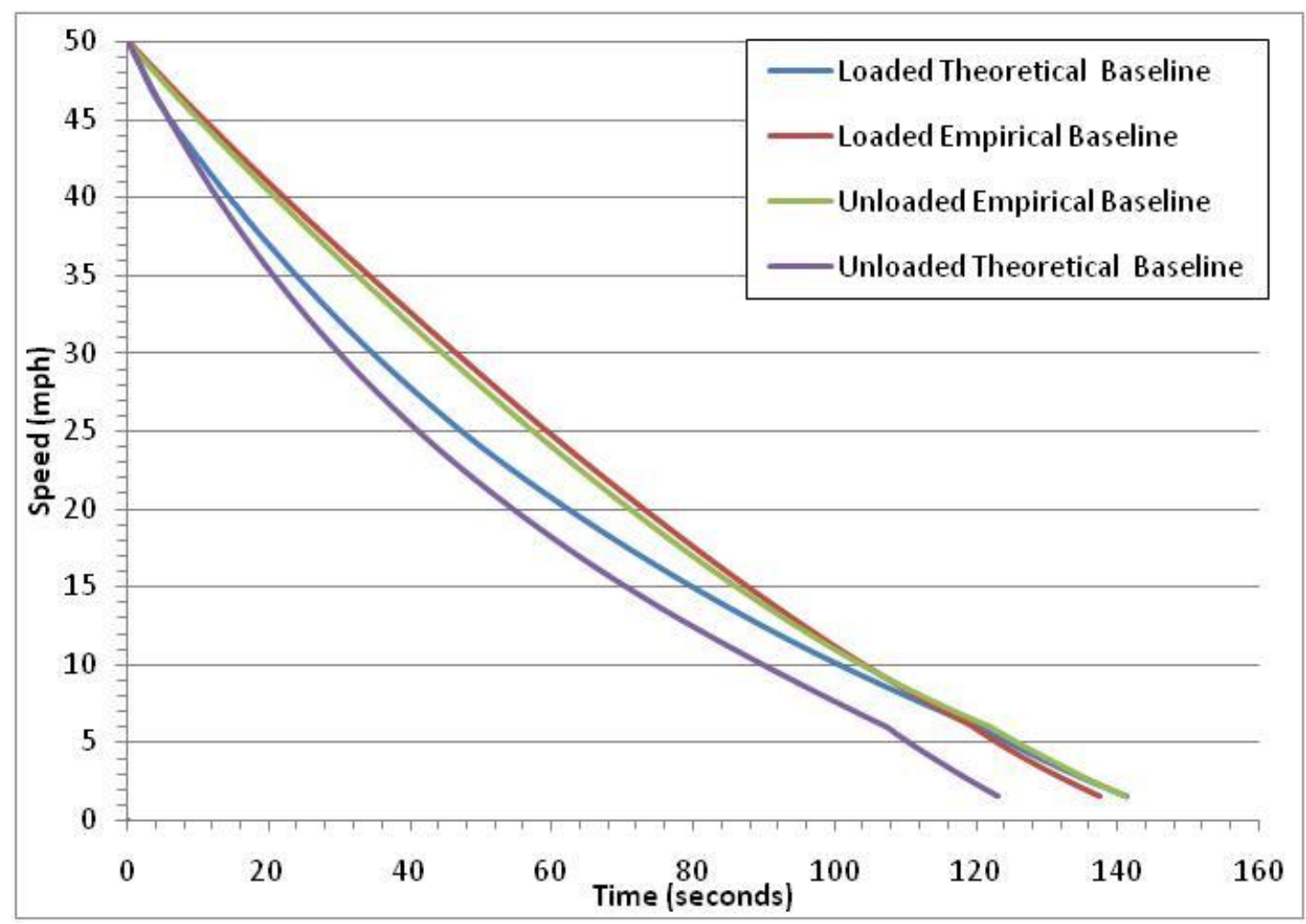

Figure 19. Comparison of chassis coastdown runs for the baseline vehicle 


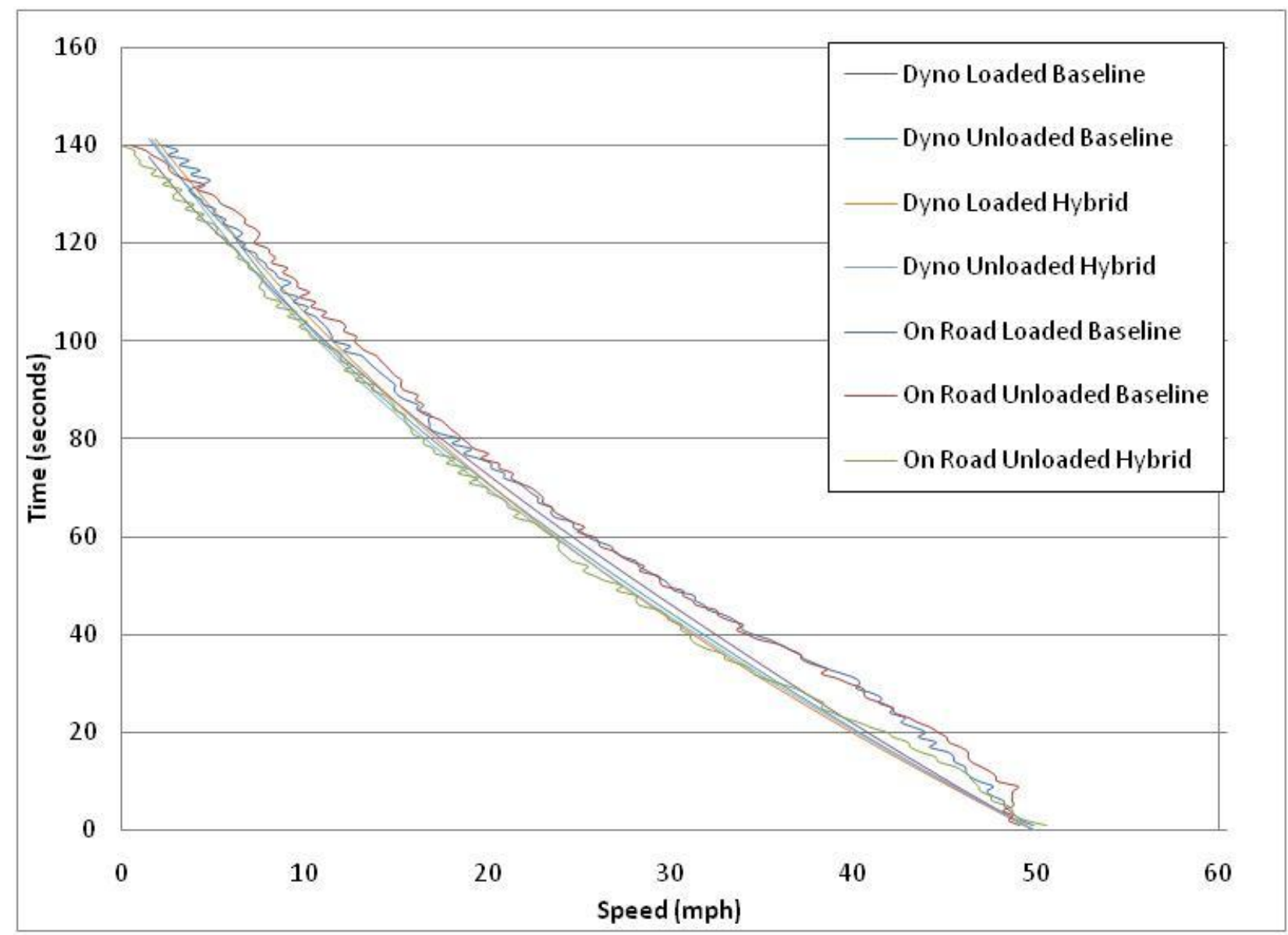

Figure 20. The comparison of the on-road coastdown versus coastdowns conducted on chassis dynamometer 


\subsection{Test Cycle Verification}

\subsection{1. $\quad$ FTP Cycles}

The tables below show the comparison of the test results from the different methods. The purpose of Table 6 and Table 7 is to verify the actual distance traveled and average speed with that of the FTP- 75 cycle. Table 6 shows the results for the baseline vehicle with different methods of coastdown used and Table 7 shows the results from the hybrid vehicle. The average speeds of each individual method were evaluated and compared with that of FTP-75 average speed. The difference was relative small with the largest being $1.16 \%$ for the theoretical loaded coastdown method. The average distances traveled for each individual method were slightly higher compared with the average speed for four methods. The total distance traveled, as shown in Table 6 and Table 7, for the four methods are less than FTP-75 test cycle which can be explained by few factors. FTP-75 test cycle was conducted by multiple drivers on the chassis dynamometer with various experience levels. When a driver is matching the speed of the vehicle with that of speed trace provided by video monitor, one must recognize when to begin accelerating the vehicle. An experienced driver would be aware of this and may be able to better follow the speed trace provided by the video monitor. 
Table 6. The FTP-75 test cycle verification for the baseline vehicle

\begin{tabular}{|l|c|c|c|c|}
\hline Method Used & $\begin{array}{c}\text { Average } \\
\text { Distance } \\
\text { Traveled }\end{array}$ & $\begin{array}{c}\% \\
\text { Difference }\end{array}$ & $\begin{array}{c}\text { Average } \\
\text { Speed }\end{array}$ & $\begin{array}{c}\% \\
\text { Difference }\end{array}$ \\
\hline Baseline Vehicle & (miles) & $(\%)$ & $(\mathrm{mph})$ & $(\%)$ \\
\hline Empirical Loaded coastdown & 11.10 & -0.58 & 21.3 & -0.57 \\
\hline Empirical Unladen coastdown & 11.11 & -0.61 & 21.3 & -0.60 \\
\hline Theoretical Loaded Coastdown & 10.91 & 1.15 & 21.0 & 1.16 \\
\hline Theoretical Unladen Coastdown & 10.73 & 2.78 & 21.1 & 0.66 \\
\hline FTP 75 Test Cycle & 11.04 & - & 21.2 & - \\
\hline
\end{tabular}

Table 7. The FTP-75 test cycle verification for the hybrid vehicle

\begin{tabular}{|l|c|c|c|c|}
\hline Method Used & $\begin{array}{c}\text { Average } \\
\text { Distance } \\
\text { Traveled }\end{array}$ & $\begin{array}{c}\% \\
\text { Difference }\end{array}$ & $\begin{array}{c}\text { Average } \\
\text { Speed }\end{array}$ & $\begin{array}{c}\text { \% } \\
\text { Difference }\end{array}$ \\
\hline Hybrid Vehicle & (miles) & $(\%)$ & $(\mathrm{mph})$ & $(\%)$ \\
\hline Empirical Loaded coastdown & 10.90 & 1.29 & 20.94 & 0.01 \\
\hline Empirical Unladen coastdown & N/A & N/A & N/A & N/A \\
\hline Theoretical Loaded Coastdown & 10.91 & 1.21 & 20.95 & 0.01 \\
\hline Theoretical Unladen Coastdown & 10.96 & 0.74 & 21.05 & 0.01 \\
\hline FTP 75 Test Cycle & 11.04 & - & 21.2 & - \\
\hline
\end{tabular}

In the appendixes, Table 23 to Table 29 each show individual tests for the methods used.

The correlation coefficient for all the tests are well above 0.99 stating that the vehicle speed was very close to the speed trace set by the test schedule. The highest standard error for all the methods was 1.47 for the hybrid vehicle, using the loaded theoretical method of coastdown as shown in

Table 28. Most of the testing was conducted by inexperienced drivers for the hybrid vehicle hence the higher standard error for all the tests compared to that of the baseline vehicle. As the inexperienced drivers became more aware of the vehicles behavior, the standard error decreased as well as the correlation coefficient. 


\subsubsection{State of Charge Results}

The service ranger software provided information SOC for the energy storage system for the hybrid vehicle during the FTP-75 tests which was used to determine if SOC correction was required for fuel economy. Table 8 provides a summary of the determination of SOC for all the runs of the FTP-75. The voltage of the battery was not monitored during the test cycles, but a nominal 340 volts DC was used for calculation of SOC correction. Heating value foe the diesel fuel was assumed to be $19,300 \mathrm{btu} / \mathrm{lb}$, a $\mathrm{k}$ factor of 3600 was used for conversion purposes. The NEC showed to be minimal compared to total energy hence the change in SOC had an insignificant effect over the course of the test cycle. Table 8 below shows the summary runs for the hybrid vehicle.

Table 8. Summary of all the runs for the hybrid vehicle and the determination of the SOC

\begin{tabular}{|r|l|l|l|r|r|r|r|r|}
\hline Run & $\begin{array}{l}\text { SOC } \\
\text { Initial }\end{array}$ & $\begin{array}{l}\text { SOC } \\
\text { Final }\end{array}$ & \multicolumn{1}{l|}{$\begin{array}{l}\text { SOC } \\
\text { (\%) }\end{array}$} & $\begin{array}{l}\text { NEC } \\
\text { (amp-hrs) }\end{array}$ & $\begin{array}{l}\text { (BTU) } \\
\text { Fuel }\end{array}$ & $\begin{array}{l}\text { (lbs) } \\
\text { Total Fuel } \\
\text { Energy }\end{array}$ & $\begin{array}{l}\text { Total Cycle } \\
\text { Energy }\end{array}$ & $\begin{array}{l}\text { \% } \\
\text { Change }\end{array}$ \\
\hline 1 & 37.6 & 32.6 & $-1.75 \mathrm{E}-03$ & 2.26 & 6.22 & $1.20 \mathrm{E}+05$ & $1.20 \mathrm{E}+05$ & -0.002 \\
\hline 2 & 31.6 & 32.8 & $4.20 \mathrm{E}-04$ & 0.542 & 6.35 & $1.23 \mathrm{E}+05$ & $1.23 \mathrm{E}+05$ & 0.000 \\
\hline 3 & 32.8 & 31.6 & $-4.20 \mathrm{E}-04$ & 0.542 & 6.26 & $1.21 \mathrm{E}+05$ & $1.21 \mathrm{E}+05$ & 0.000 \\
\hline 4 & 28.8 & 32.8 & $1.40 \mathrm{E}-03$ & 1.81 & 5.73 & $1.11 \mathrm{E}+05$ & $1.11 \mathrm{E}+05$ & 0.002 \\
\hline 5 & 32.8 & 32.8 & $0.00 \mathrm{E}+00$ & 0.000 & 5.73 & $1.11 \mathrm{E}+05$ & $1.11 \mathrm{E}+05$ & 0.000 \\
\hline 6 & 28.8 & 33.6 & $1.68 \mathrm{E}-03$ & 2.17 & 5.82 & $1.12 \mathrm{E}+05$ & $1.12 \mathrm{E}+05$ & 0.002 \\
\hline 7 & 33.6 & 34.8 & $4.20 \mathrm{E}-04$ & 0.542 & 5.69 & $1.10 \mathrm{E}+05$ & $1.10 \mathrm{E}+05$ & 0.000 \\
\hline 8 & 34.8 & 33.6 & $-4.20 \mathrm{E}-04$ & 0.542 & 5.60 & $1.08 \mathrm{E}+05$ & $1.08 \mathrm{E}+05$ & -0.001 \\
\hline
\end{tabular}




\subsection{Power Absorber Settings Based on the Method of Coastdown}

\subsubsection{Hybrid Vehicle}

PA settings during the testing of the hybrid vehicle can be seen in Table 9,

Table 10, and Table 11. The PA setting for the loaded theoretical method is more aggressive than that of the loaded empirical method during the FTP-75 test schedule. This also can be noticed in Figure 21 and Figure 22, especially at high speeds. This can be explained by the fact that the theoretical coastdown assumes a higher $\mathrm{C}_{\mathrm{d}}$ and $\mathrm{C}_{\mathrm{rr}}$ for this particular vehicle as shown in Table 5. On average, the loaded theoretical method is simulating $27.47 \%$ more load on the vehicle compared to the empirical method for this vehicle application.

Table 9. The PA setting for the hybrid vehicle during the FTP-75 test schedule using method of theoretical loaded coastdown

\begin{tabular}{|l|c|c|c|}
\hline & Max (HP) & Min (HP) & Average (HP) \\
\hline PA Setpoint & 59.6 & 0.00 & 7.27 \\
\hline PA Actual & 59.5 & 0.00 & 7.28 \\
\hline Added Motor Power & 0.073 & 0.00 & 0.00 \\
\hline
\end{tabular}

Table 10. The PA setting for the hybrid vehicle during the FTP-75 test schedule using method of empirical loaded coastdown

\begin{tabular}{|l|c|c|c|}
\hline & Max (HP) & Min (HP) & Average (HP) \\
\hline PA Setpoint & 19.9 & 0.00 & 5.36 \\
\hline PA Actual & 23.7 & 0.00 & 5.28 \\
\hline Added Motor Power & 0.00 & 0.00 & 0.00 \\
\hline
\end{tabular}




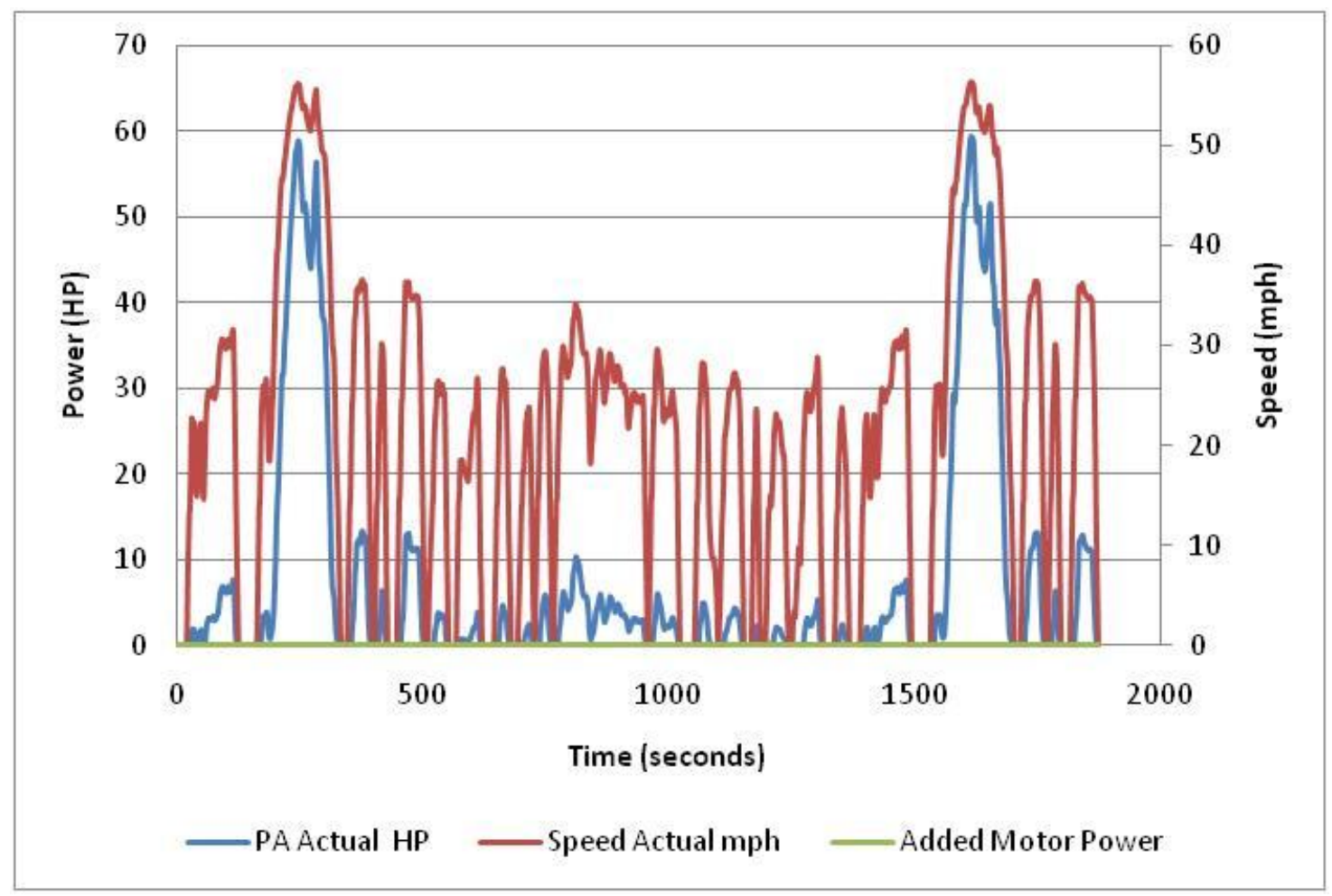

Figure 21. The average of PA settings and the speeds of hybrid vehicle with loaded theoretical method during FTP-75 test schedule, and no motor power was needed during idle periods. 


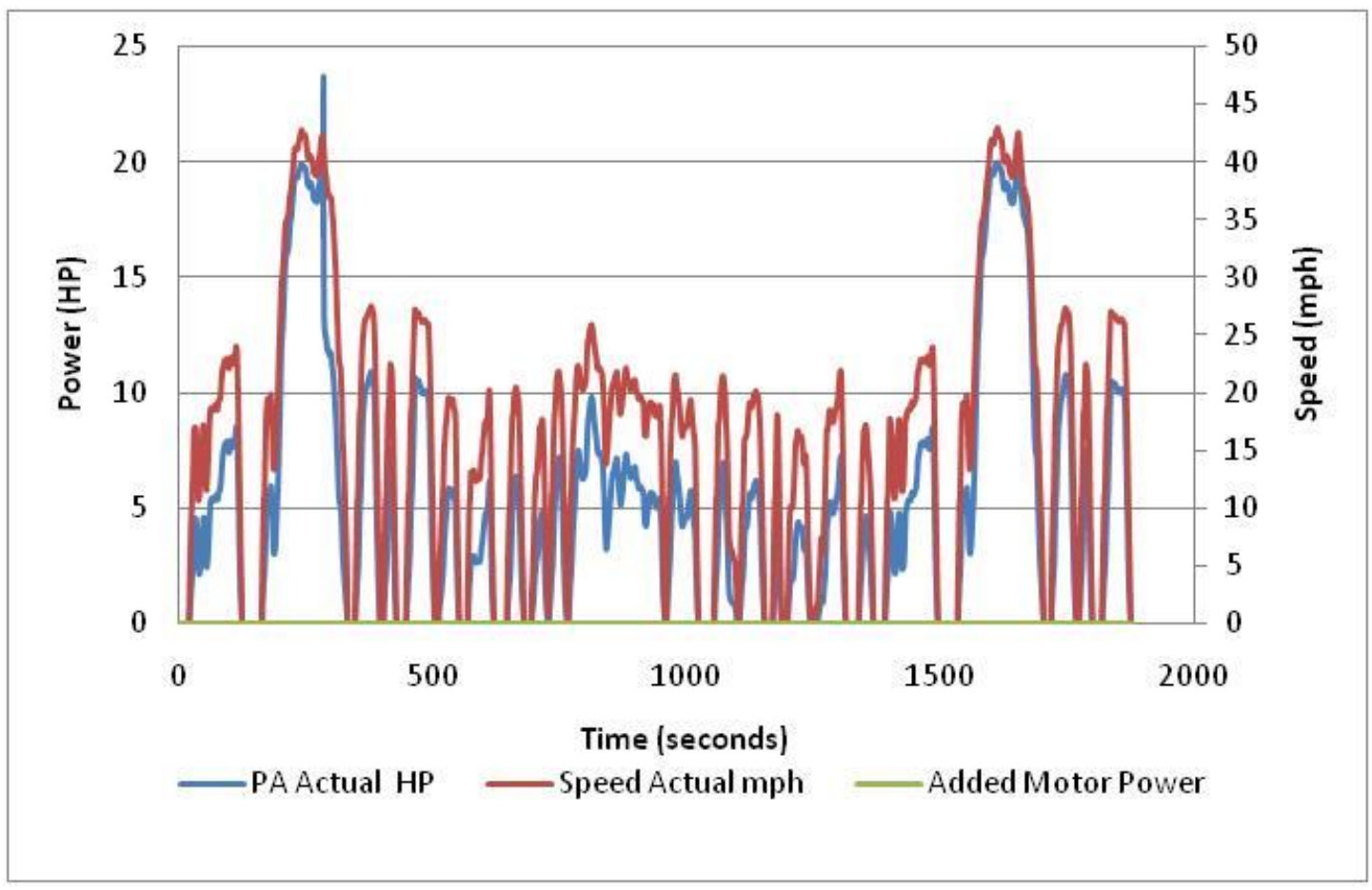

Figure 22. The average of the PA settings and the speeds of the hybrid vehicle with loaded empirical method during FTP-75 test schedule, and no motor power were needed during idle periods

A noticeable change can be seen between the loaded coastdown and the unladen coastdown methods. Table 11 and Figure 23 show the results of the unladen theoretical coastdown method during FTP-75 test schedule. On average, the PA setting for the loaded coastdown was $8 \%$ higher than that of the unladen coastdown method. The added test weight would only influence the loading of PA's from rolling resistance due to the fact the load was placed inside the vehicle and had no effect on the frontal area of the vehicle during the coastdown period. Unfortunately, a valid conclusion could not be drawn for the loading of the empirical method due to the data mismanagement of the empirical unladen coastdown tests. But an engineering estimate could was drawn from the data available from the baseline vehicle. Using extrapolation and the relationship between the loaded and unladen baseline PA results, the setting for the unladen empirical coastdown could be around 3.77 HP. Some causes of error that 
contribute to this conclusion include losses from the drivetrain of the hybrid vehicle, limited number of runs available for the regression analysis, and day to day variation in weather.

Table 11. The PA setting for the hybrid vehicle during the FTP-75 test schedule using method of theoretical unladen coastdown

\begin{tabular}{|l|c|c|c|}
\hline & Max (HP) & Min (HP) & Average (HP) \\
\hline PA Setpoint & 54.2 & 0.00 & 6.69 \\
\hline PA Actual & 54.1 & 0.00 & 6.70 \\
\hline Added Motor Power & 0.00 & 0.00 & 0.00 \\
\hline
\end{tabular}

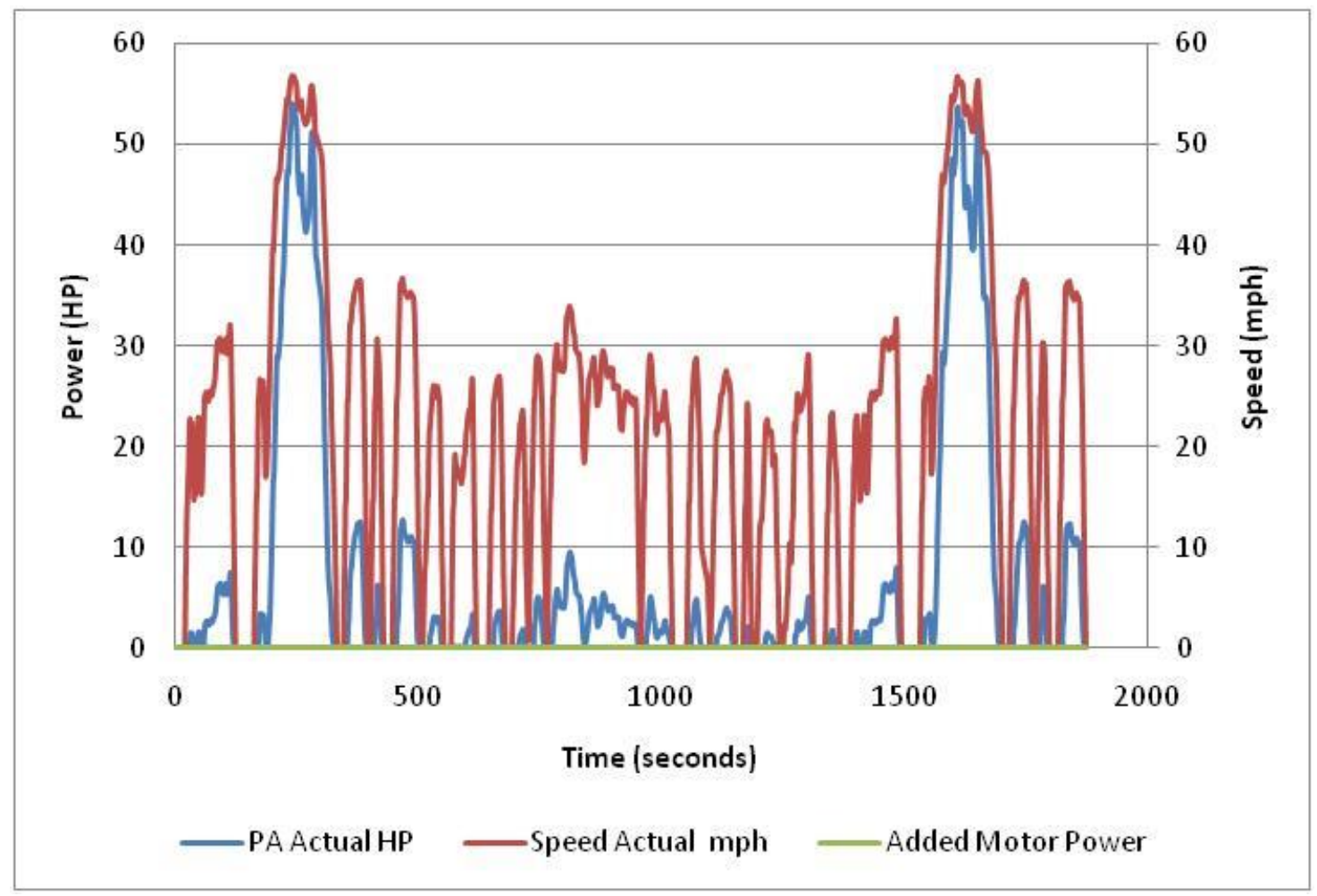

Figure 23. The average of the PA settings and speeds of hybrid vehicle with Curb theoretical method, no added motor power was required during idling periods

Table 30, Table 31 and Table 32 show the analysis of power data for the hybrid vehicle. The runs from the analysis show a correlation coefficient of 0.99 or greater except for run 34 where the correlation coefficient is 0.874 . These numbers are acceptable and verify that the set 
point numbers match the actual points. For run 34, where the correlation coefficient was 0.874 and standard error of 1.901 was due to no response in the controller in the analytical laboratory. The no response period lasted about 40 to 45 seconds of the whole cycle, which is less than 2.4 $\%$ of the whole cycle.

\subsubsection{Baseline Vehicle}

Table 12 and Table 13 show the PA settings for the theoretical loaded method and empirical loaded method, respectively, from the FTP-75 test cycle. The theoretical method is simulating $44.86 \%$ higher road loads than that of empirical method for the baseline vehicle. Figure 24 and Figure 25 show the power, speed and motor torque over the course of the test cycle. As stated before, the theoretical method shows, on average, higher road loads than that of empirical method.

Table 12. The PA setting for the baseline vehicle during the FTP-75 test schedule using method of theoretical loaded coastdown

\begin{tabular}{|l|c|c|c|}
\hline & Max (HP) & Min (HP) & Average (HP) \\
\hline PA Setpoint & 79.7 & 0.00 & 10.8 \\
\hline PA Actual & 102 & 0.00 & 10.9 \\
\hline Added Motor Power & 0.00 & 0.00 & 0.00 \\
\hline
\end{tabular}

Table 13. The PA setting for the baseline vehicle during the FTP-75 test schedule using method of empirical loaded coastdown

\begin{tabular}{|l|c|c|c|}
\hline & Max (HP) & Min (HP) & Average (HP) \\
\hline PA Setpoint & 21.8 & 0.00 & 5.98 \\
\hline PA Actual & 23.36 & 0.00 & 6.01 \\
\hline Added Motor Power & 0.00 & 0.00 & 0.00 \\
\hline
\end{tabular}




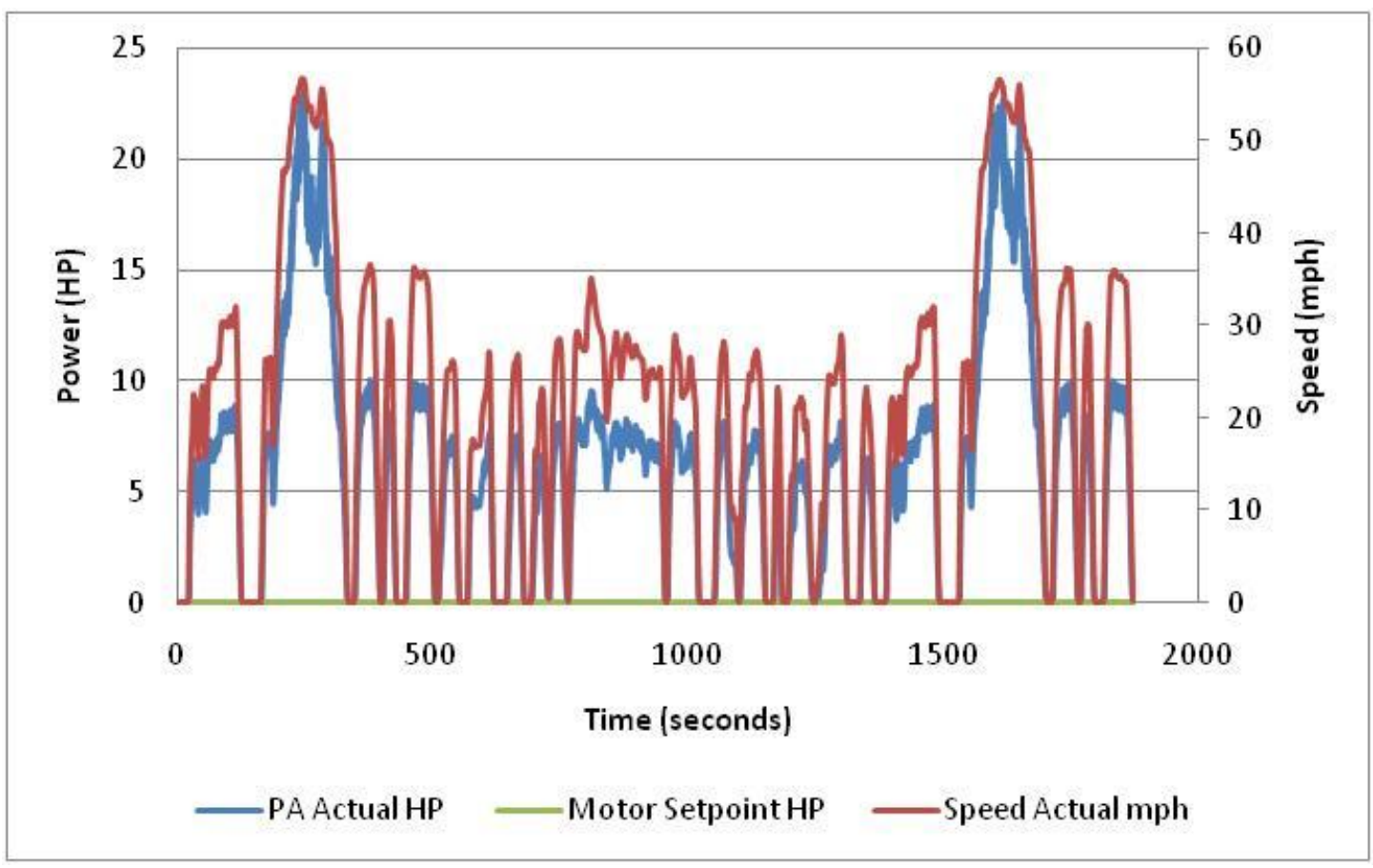

Figure 24. The average of the PA settings and speeds of baseline vehicle with loaded empirical method, no added motor power was required during idling periods

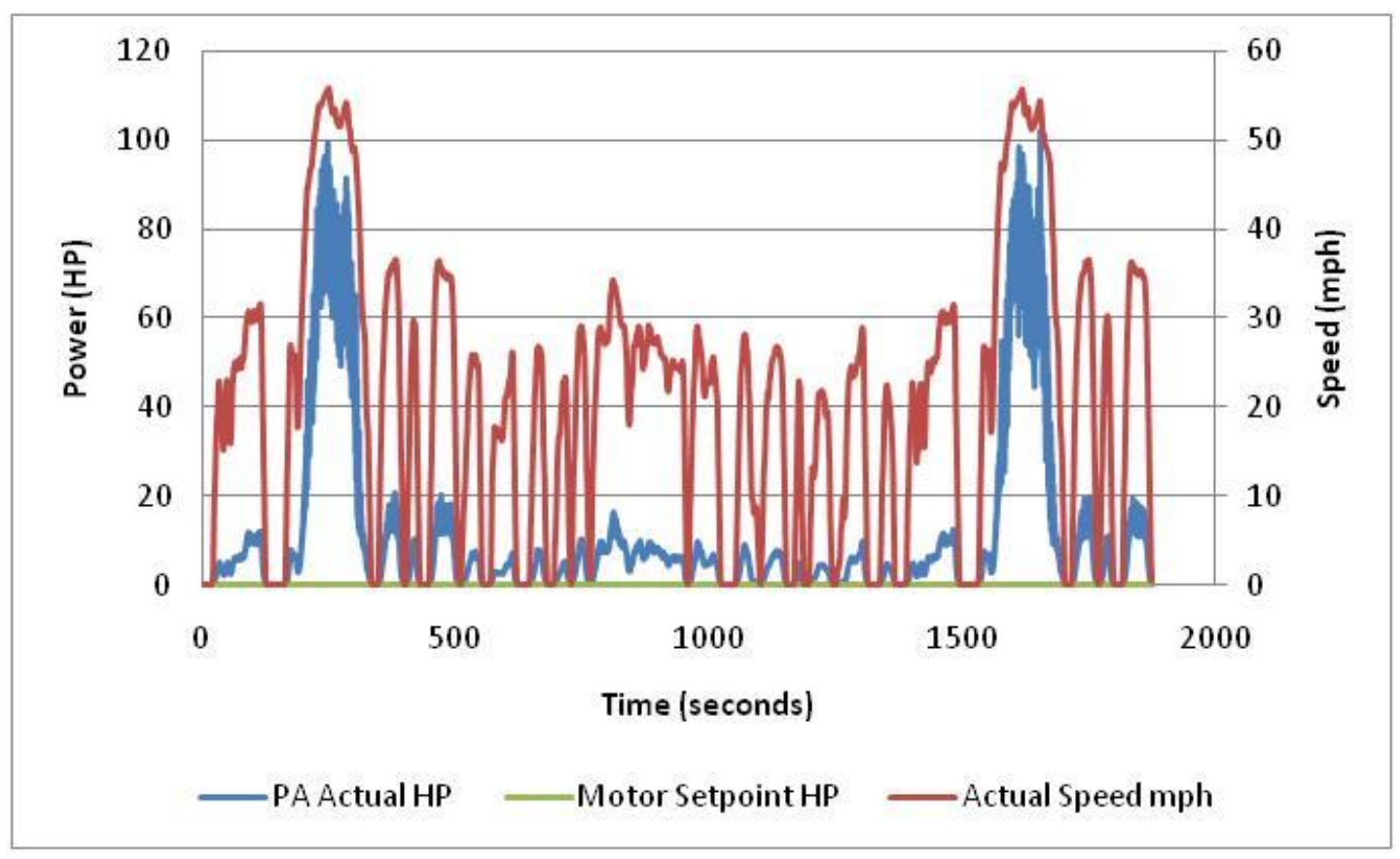

Figure 25. The average of the PA settings and speeds of baseline vehicle with loaded theoretical method, no added motor power was required during idling periods 
Table 33 and Table 34 show the analysis of the power data for the baseline vehicle. The loaded empirical method had reasonable numbers with a correlation coefficient above 0.980 and a standard error of less than 1.00 for all three runs shown in Table 34 . The standard error of the loaded theoretical method was 5.75 for run 3, being the highest. The correlation coefficient for all the runs was still above 0.980 .

Table 14 and Table 15 show the PA settings for the theoretical unladen method and the empirical unladen method for the baseline vehicle. The PA response of the theoretical unladen coastdown was higher than the empirical. The PA actual was, on average, $45 \%$ higher setting than that of PA setpoint. On average, the PA actual setting for the empirical method was $79 \%$ less than that of the PA actual setting for the theoretical method. Comparing the results for the baseline vehicle from the empirical method, the test weight added $25 \%$ load to truck from the PA. Figure 26 and Figure 27 show the power, speed and motor torque over the course of the test cycle. Both methods show to have the motor torque added during the idle periods of the test. But with such low values that it can be considered as noise.

Table 14. The PA settings for the baseline vehicle during the FTP-75 test schedule using method of theoretical unladen coastdown

\begin{tabular}{|l|c|c|c|}
\hline & Max (HP) & Min (HP) & Average (HP) \\
\hline PA Setpoint & 82.2 & 0.00 & 11.6 \\
\hline PA Actual & 103 & 0.00 & 21.1 \\
\hline $\begin{array}{l}\text { Added Motor } \\
\text { Power }\end{array}$ & 0.008 & 0.00 & 0.000 \\
\hline
\end{tabular}

Table 15. The PA setting for the baseline vehicle during the FTP-75 test schedule using method of empirical unladed coastdown

\begin{tabular}{|l|c|c|c|}
\hline & Max (HP) & Min (HP) & Average (HP) \\
\hline PA Setpoint & 16.6 & 0 & 4.4 \\
\hline PA Actual & 18 & 0 & 4.5 \\
\hline $\begin{array}{l}\text { Added Motor } \\
\text { Power }\end{array}$ & 0.055 & 0 & 0.002 \\
\hline
\end{tabular}




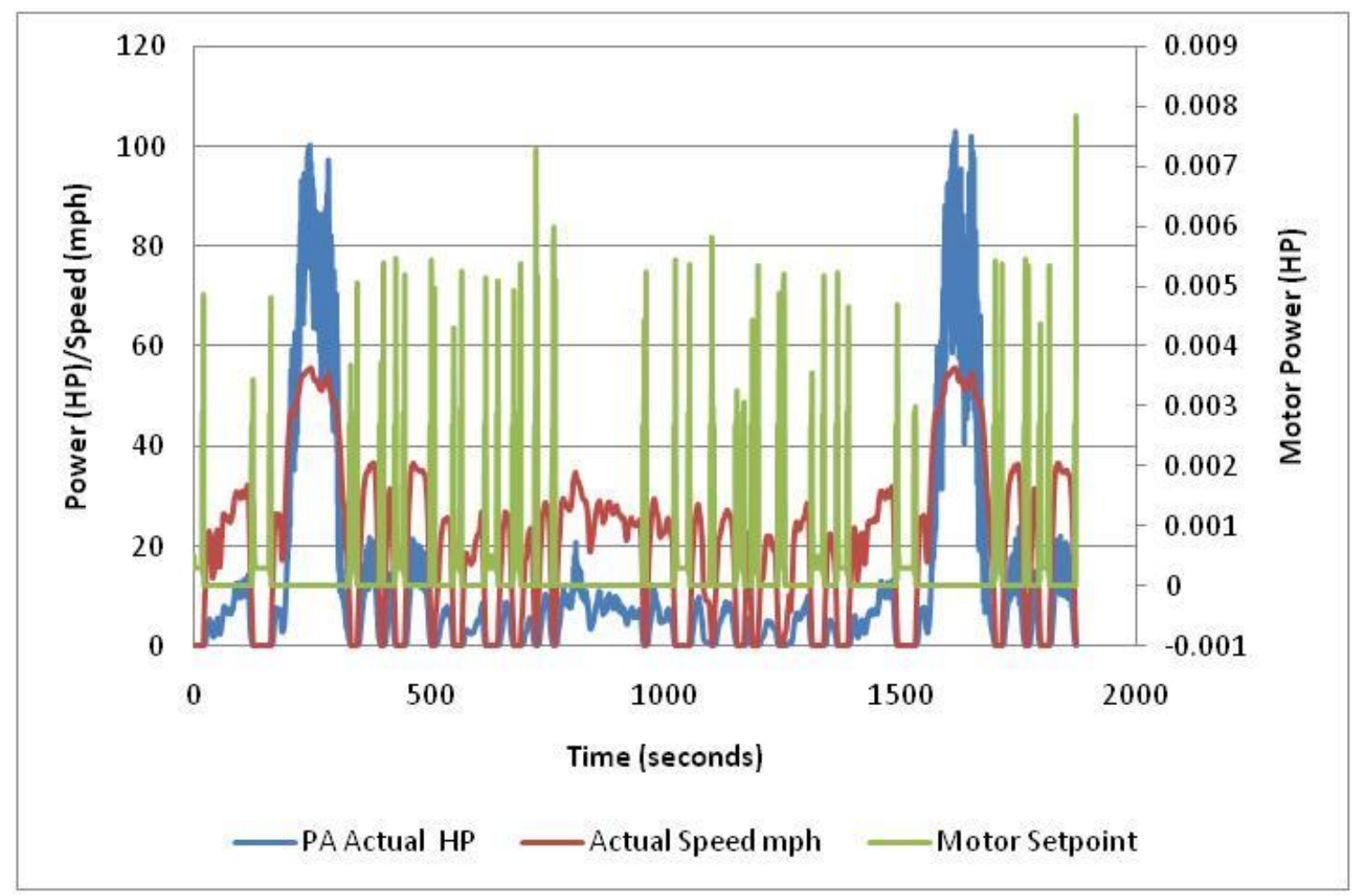

Figure 26. The average of the PA settings and speeds of baseline vehicle with unladen theoretical method during FTP-75 test schedule

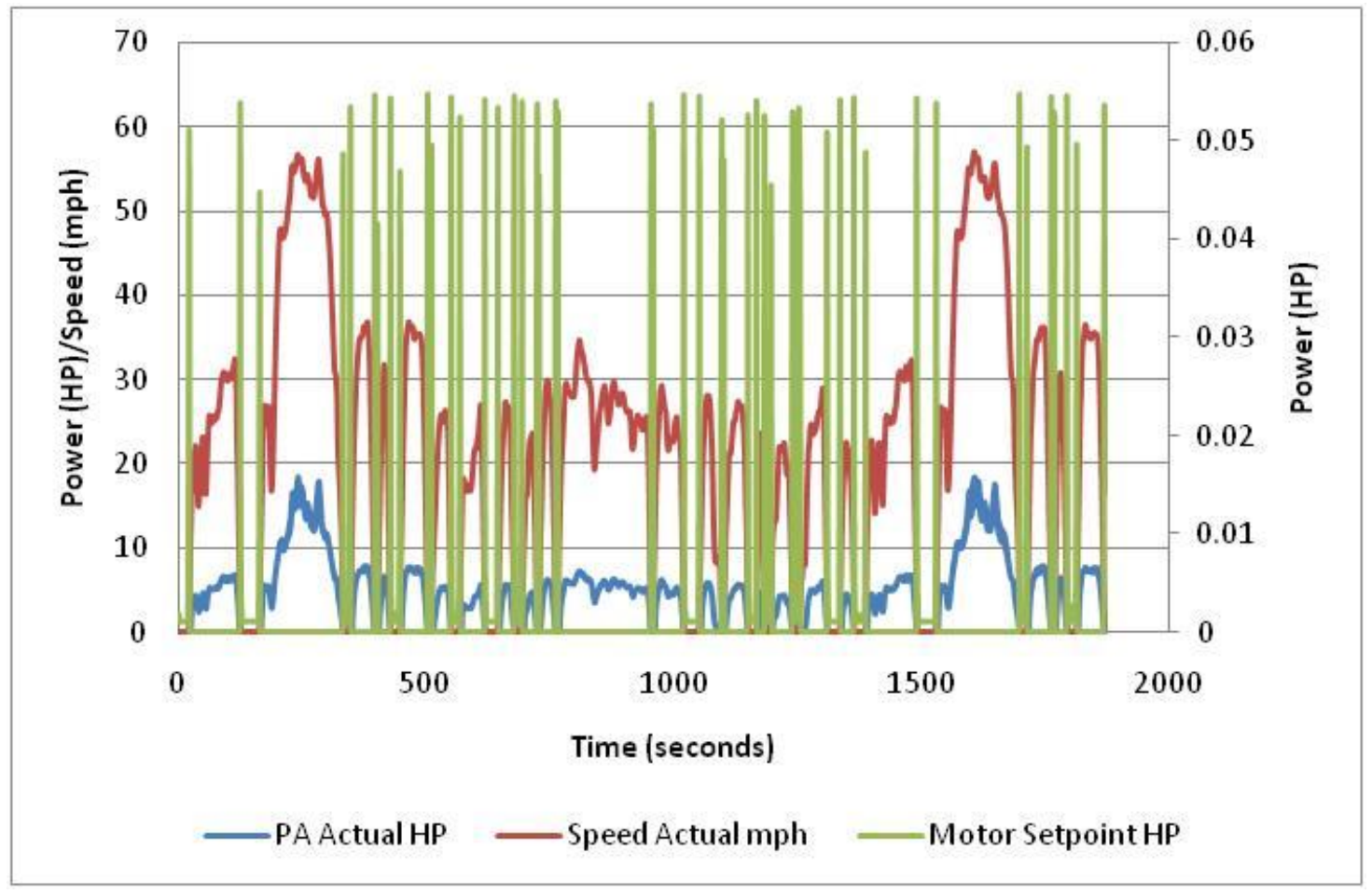

Figure 27. The average of the PA settings and speeds of baseline vehicle with unladen empirical method during FTP-75 test schedule 
Table 33, Table 34, Table 35, and Table 36 show the analysis of power data for the baseline vehicle. The standard errors for the empirical method were all around 0.50 with the correlation coefficient of 0.99 . For the theoretical method the standard error was around 5.0 for

all the runs; being ten times higher than empirical method. The variation in the power regression could be explained by few things, as it was explained by Clark et al. [28]. The difference in regression could be explained by the difference in the drivers' experiences. The driver finds a need to remain close the trace line, leading some drivers to employ severe pedal position changes. Yet other drivers feel that the pedal position changes should be subtle, and compromise at the best they can between maintaining schedule speed and avoiding harsh control.

\subsection{Fuel Economy and Work}

Table 16 and Figure 28 below show the summary for both methods and both vehicles. The loaded hybrid configuration and unladen baseline configuration both showed an improvement in fuel economy from the empirical method compared to the theoretical method. However, the loaded baseline configuration showed a 5.31\% difference from the empirical method compared to the theoretical method. Conditions that may have caused this may be weather conditions, error in the regression analysis, or tire rolling resistance. As mentioned before, a $10.0 \%$ change in rolling resistance leads to a change of $2.00 \%$ in fuel economy. A conclusion could not be drawn for the method of empirical unladen hybrid.

Table 17 shows the comparison of fuel economy for the hybrid vehicle to baseline vehicle for each type of road load implementation. For the theoretical method of coastdowns, the hybrid vehicle showed a $24.8 \%$ improvement over the baseline vehicle with additional test 
weight. For the loaded empirical method, the hybrid vehicle showed a $34.4 \%$ improvement over the baseline vehicle. Without the additional test weight, there was a $27.7 \%$ improvement in fuel economy for the hybrid vehicle compare to the baseline vehicle. The $10.0 \%$ between the two methods could be combination of things as previously investigated. The main contributor could be the aggressive PA settings based on the theoretical coastdown method used. Another could include weather conditions during the empirical coastdowns, tire temperature, tire pressure, and losses in the drivetrain which is not accounted for in the road load equation.

Table 16. Summary of the average fuel economy for both methods and vehicles

\begin{tabular}{|l|c|c|c|}
\hline Fuel Economy & Empirical Method (mpg) & Theoretical Method (mpg) & \% Difference \\
\hline Loaded Hybrid & 13.5 & 12.4 & 8.15 \\
\hline Loaded Baseline & 8.86 & 9.33 & 5.31 \\
\hline Unladen Hybrid & N/A & 13.5 & N/A \\
\hline Unladen Baseline & 12.0 & 9.76 & 18.7 \\
\hline
\end{tabular}

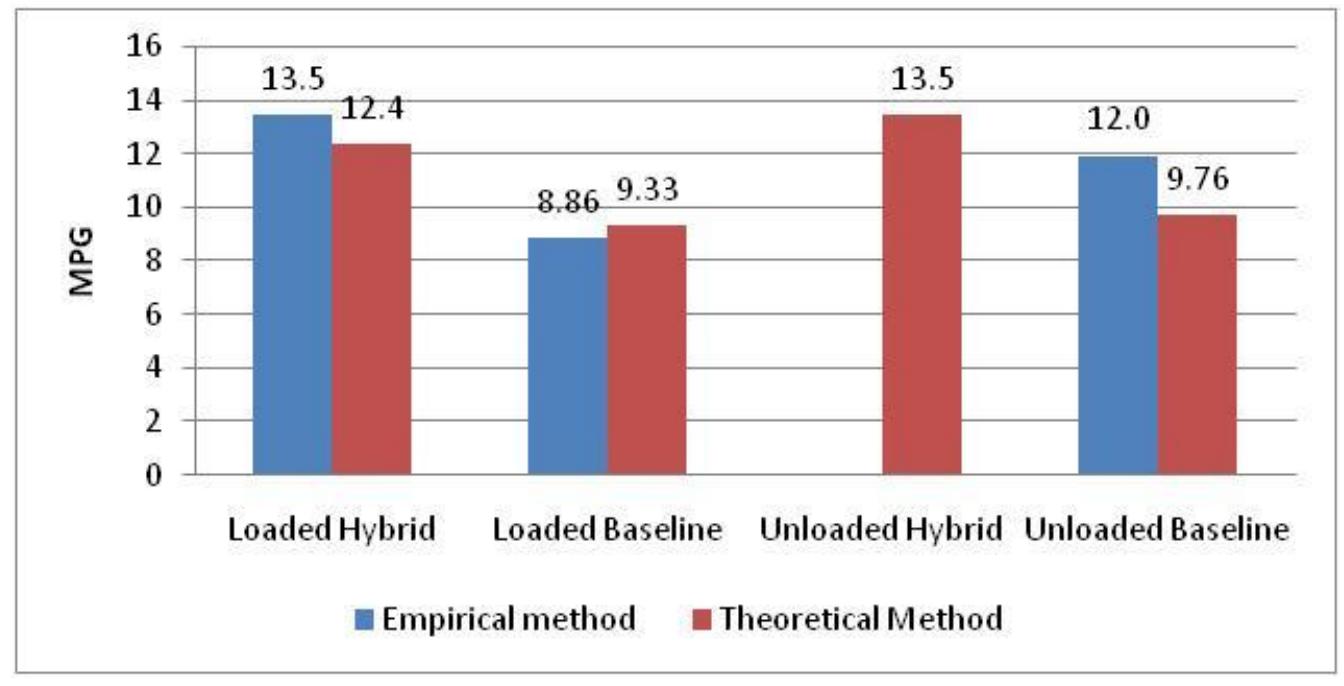

Figure 28. Fuel economy comparison of both vehicles for each configuration 
Table 17. Fuel economy comparisons of the loaded vehicles with different methods of road load implementation

\begin{tabular}{|l|c|c|r|}
\hline MPG & $\begin{array}{l}\text { Loaded } \\
\text { Hybrid }\end{array}$ & $\begin{array}{l}\text { Loaded } \\
\text { Baseline }\end{array}$ & \% Difference \\
\hline Empirical method & 13.5 & 8.86 & 34.4 \\
\hline $\begin{array}{l}\text { Theoretical } \\
\text { Method }\end{array}$ & 12.4 & 9.33 & 24.8 \\
\hline
\end{tabular}

Table 18 and Figure 29 show the summary of work done during the FTP-75 test cycle. The reason the data is illustrated in units of work per distance traveled is to balance results. In depth results of all the runs for both the empirical method and theoretical method are shown Table 37 to Table 43. The distance traveled by the vehicle may vary run to run, due to driver's ability to follow the trace on the monitor. If the driver is unable to follow the trace, the distance traveled may be less that of the cycle, but the driver over shoots the trace than the distance traveled is more than that set by the test cycle. Work per distance proportions all the runs accordingly. Table 18 below shows that the empirical method was $2.29 \%$ different for the two loaded vehicles compared to the $13.0 \%$ of the theoretical method. For the theoretical method, it shows a $15.0 \%$ between the unladen vehicles.

Table 18. Summary of work per distance traveled for both methods and vehicles.

\begin{tabular}{|l|c|c|c|}
\hline $\begin{array}{l}\text { Work Per } \\
\text { Distance }\end{array}$ & $\begin{array}{c}\text { Empirical Method } \\
(\mathrm{whp} / \mathrm{hr} \mathrm{mi})\end{array}$ & $\begin{array}{c}\text { Theoretical Method } \\
(\mathrm{whp} / \mathrm{hr} \mathrm{mi})\end{array}$ & \% Difference \\
\hline Loaded Hybrid & 0.829 & 0.922 & 28.3 \\
\hline Loaded Baseline & 0.848 & 1.06 & 25.0 \\
\hline Unladen Hybrid & N/A & 0.843 & N/A \\
\hline Unladen Baseline & 0.695 & 1.00 & 43.9 \\
\hline
\end{tabular}




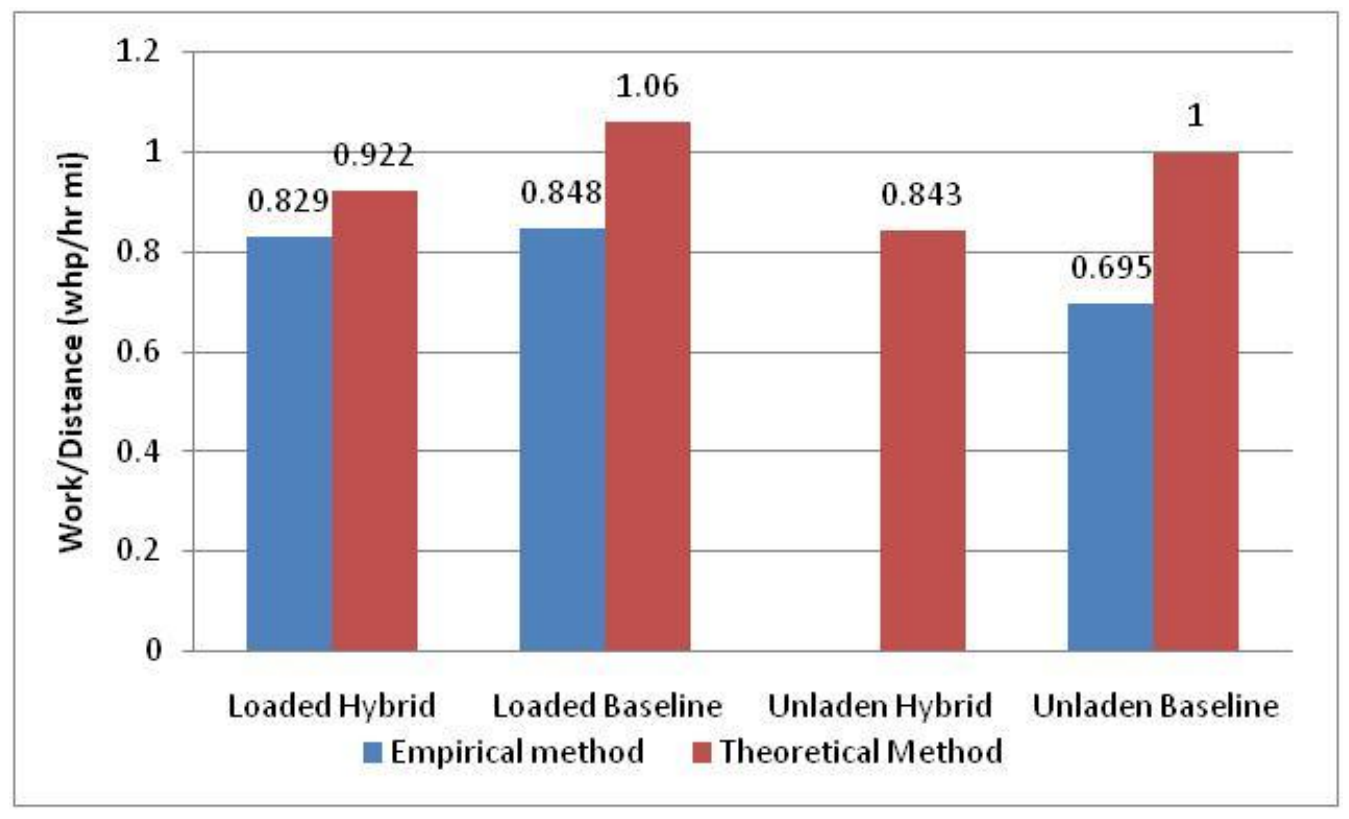

Figure 29. Work per distance comparison for both vehicles for all four configurations

Table 19 below shows the comparison of work per distance for both vehicles with respect to each method. This is an interesting statistic, as it shows if the full capability of the hybrid vehicle was used during the test cycle. The theoretical method showed a difference of $2.29 \%$ between the two vehicles compared to the $15.0 \%$ from the empirical method. The result of this concludes that the engine was operating on high loads during the test cycle due to the implementation of more aggressive PA settings from the theoretical method.

Table 19. Comparison of the Vehicles with different coastdown method under loaded condition.

\begin{tabular}{|c|c|c|c|}
\hline & $\begin{array}{l}\text { Loaded Hybrid } \\
\text { (whp/hr mi) }\end{array}$ & $\begin{array}{l}\text { Loaded Baseline } \\
\text { (whp/hr mi) }\end{array}$ & \% Difference \\
\hline Theoretical Method & 0.829 & 0.848 & 2.29 \\
\hline Empirical method & 0.922 & 1.06 & 15.0 \\
\hline
\end{tabular}




\section{Conclusion and Recommendations}

\subsection{Conclusion}

The primary goal of this study is to compare and contrast an empirical method of coastdowns to a theoretical method through Federal Test Procedure 75 cycle using WVU's THDEVETL and two United States Postal Service (USPS 2-ton) medium duty trucks. For both of the vehicles, the theoretical method showed higher road loads than that of the empirical method. As shown in the empirical and the theoretical model, using regression analysis, the $C_{d}$ from the theoretical method estimates a value of 0.735 as compared to that of 0.669 from the empirical method. From the theoretical model, $\mathrm{C}_{\mathrm{rr}}$ equated to 0.00937 compare to the empirical model which equated to 0.0139 .

The rolling resistance based on the empirical model was much higher than it should be, due to the fact it took into the account the losses in the drivetrain as well as weather conditions at the time. The rolling resistance provided by the tire companies may not be the same as the ones experienced on road, where the ambient conditions, road material, and variation in weight have an influence. The information provided by the GPS unit showed to have large variation in altitude and speed in small increments of time. Even though the vehicle was driven in the opposite direction to minimize altitude change, the variation still existed from the GPS unit. Figure 10, Figure 11, and Figure 12 show the raw data from the GPS unit. Although there are areas where the speed of the vehicle was constant, the figure shows variation in speed was present. 
With the theoretical method, the hybrid vehicle showed a $24.8 \%$ improvement in fuel economy as compared to $34.4 \%$ obtained in the empirical method. Fuel economy comparison showed to be affected the most with change of $10 \%$ between the two methods. If a more aggressive road load implementation was used for the comparison of the hybrid vehicle versus the baseline vehicle, the results might be misleading.

Table 19 shows how effective the hybrid system was for each method. For the theoretical method, work per distance traveled proved to be $2.29 \%$ between the hybrid vehicle and the baseline vehicle. For the empirical method, work per distance traveled proved to be $15.0 \%$ between the two vehicles. The hybrid USPS step van proved to be more effective under the empirical method of road load implementation.

\subsection{Recommendations}

For future work, a better method of logging of speed and altitude is inevitable. Rather than relying on the GPS unit to record speed, use of the CAN adapter to record data from the ECU could provide stable results. Due to time constraints and availability of the vehicles, coastdowns were conducted on different days of the month which in turn caused some error in the results from variation in weather conditions. If possible, a recommendation is to conduct all the on road coastdowns on same day or days where the weather conditions are similar.

To further decrease variation in the power and speed from the FTP cycle, having an experienced driver to conduct all of the test schedules would be beneficial. The SOC correction calculated used an instantaneous result from the service ranger software. It would be interesting to see how the hybrid system behaves during a test cycle by monitoring the voltage and SOC of the energy storage system throughout the whole test. 


\section{References}

1 Ferguson, D. H., Gautam, M., Want, W. G., Clark, N. N., Lyons, D. W., Bata, R. M., Palmer, G. M., Katragadda, S. "Exhaust Emissions from In-Use Heavy Duty Vehicles Tested on Transportable Transient Chassis Dynamometer.” SAE Technical Paper. SAE 922436.

2 Clark, N. N., Xie, W., Gautam, M., Lyons, D. W., Norton, P., Balon, T. "Hybrid DieselElectric Heavy Duty Bus Emissions: Benefits of Regeneration and need for State of Charge Correction.” SAE Technical Paper. SAE 2000-01-2955.

3 Clark, N. N., Gautam, M., Wayne, W. S., Riddle, W., Nine, R. D., Lyons, D. W., Xu, S. "Examination of a Heavy-Duty Diesel Truck Chassis Dynamometer Schedule." SAE Technical Paper. SAE 2004-01-2904.

4 Gautam, M., Clark, N., Riddle, W., Nine, R., Wayne, W. S., Maldonado, H., Agrawal, A., Carlock, M. "Development and Initial use of a Heavy-Duty Diesel Truck Test Schedule for Emissions Characterization." SAE Technical Paper. SAE 2002-01-1753.

5 Wayne, S., W., Clark, N. N., Nine, R. D., Elafante, D. “A comparison of emissions and fuel economy from hybrid-electric and conventional-drive transit buses." Energy and Fuels. April 2003: pages 257-270.

6 Clark, N. N., Atkinson, R. J., Luzader, Z., VanScoy, F. L., Baker, V., Chandler, J., "Development Of An Interface Method For Implementing Road Grade In Chassis Dynamometer Testing." 2004 Fall Technical Conference of the ASME Internal Combustion Engine Division. ICEF2004-896.

7 Thompson, G. D., "Prediction of Dynamometer Power Absorption to Simulate Light Duty Truck Road Load.” SAE Technical Paper. SAE 770844.

8 Mechanical and Aerospace Department, Class 593H. "Special Topic: Conventional and Hybrid Vehicle Emissions.” Fall semester of 2008. Prof. Gregory Thompson.

9 Mechanical and Aerospace Department, Class 493H. "Special Topic: Engine Combustion and Emissions.” Spring Semester of 2008. Prof. Hailin Le.

10 Takada, Y., Ueki, S., Saito, A. "Investigation into Fuel Economy and $\mathrm{NO}_{\mathrm{x}}$ Emission of Light Duty Hybrid Truck in Real Traffic Conditions.” SAE Technical Paper. SAE 200501-0265.

11 O’Keefe, M. P., Simpson, A., Kelly., K. J., Pedersen, D. S. "Duty Cycle Characterization and Evaluation Towards Heavy Hybrid Vehicle Applications." SAE Technical Paper. SAE 2007-01-0302.

12 Hosoya, R., Yamaguchi, K., "Development of the new light-duty hybrid truck." $\underline{\text { SAE }}$ Technical Paper. SAE 2007-01-3478.

13 Francfort, J., Karner, D., Harkins, R., Tardiolo, J. "Hybrid Electric Vehicle Fleet and Baseline Performance Testing." SAE Technical Paper. SAE 2006-01-1267. 
14 McKain, D. L., Clark, N., Balon, T., Moynihan, P., Webb, T., Lynch, S., "Characterization of emissions from hybrid-electric and conventional transit Buses." $\underline{\mathrm{SAE}}$ Technical Paper. SAE 2000-01-2011.

15 SAE International: Surface vehicle recommended practice. "Recommended Practice for Measuring Fuel Economy and Emissions of Hybrid-Electric and Conventional HeavyDuty Vehicles.” Issued September 2002., SAE J2711.

16 Yasin, T. P. “The Analytical Basis of Automobile Coastdown Testing." $\underline{\text { SAE Technical }}$ Paper. SAE 780334.

17 Buckley, F. T., Marks, C. H., Walston, W. H. “Analysis of Coast-Down Data to Assess Aerodynamic Drag Reduction on Full-Scale Tractor-Trailer Trucks in Windy Environments." SAE Technical Paper. SAE 760850.

18 Passmore, M. A., Good, G. M. L. "A Detailed Drag Study Using the Coastdown Method." SAE Technical Paper. SAE 940420.

19 Walston, W. H., Buckley, F. T., Marks, C. H., "Test Procedure for the Evaluation of Aerodynamic Drag on Full-Scale Vehicles in Windy Environments." SAE Technical Paper. SAE 760106.

20 White, R. A., Korst, H. H. "The Determination of Vehicle Drag Contributions from Coast-Down Tests.” SAE Technical Paper. SAE 720099.

21 White, R. G. S. “A Method of Estimating Automobile Drag Coefficients.” SAE Technical Paper. SAE 690189.

22 Charles, T. J., Smith, J. R., Potter, D. S. "Tire Rolling Resistance - A Speed Dependent Contribution." SAE Technical Paper. SAE 780255.

23 Dayman, B. J. “Tire Rolling Resistance Measurements From Coast-Down Tests." $\underline{\text { SAE }}$ Technical Paper. SAE 760153.

24 DaRaad, L. W. "The Influence of Road Surface Texture on Tire Rolling Resistance." SAE Technical Paper. SAE 780257.

25 DieselNet. 2009 Ecopoint Inc. World Wide Web, http://www.dieselnet.com/ . February 4, 2009.

26 Environmental Protection Agency 1065: Washington, DC, World Wide Web. http://www.epa.gov. August 12, 2008.

27 Wu, Y., Carder, D., Shade, B., Atkinson, R., Clark, N., Gautam, M. “A CFR1065compliant transportable/on-road low emissions measurement laboratory with dual primary full-flow dilution tunnels.” ASME Technical Paper. ICES2009-76090.

28 Clark, N. N., McKain, L. D. "Speed and Power Regressions for Quality Control of Heavy Duty Vehicle Chassis Dynamometer Research.” SAE Technical Paper. SAE 1999-010614.

29 Wang, W. G., Bata, R. M., Lyons, D. W., Clark, N. N., Palmer, M. G., Gautam, M., Howell, A. D., Rapp., B. L. "Transient Response in a Dynamometer Power Absorption System.” SAE Technical Paper. SAE 920252. 
30 Google Maps. Map data $@ 2009$, Maponics, Tele Atlas. http://maps.google.com/maps . $\underline{02 / 20 / 2009 .}$.

31 HeyWhatsThat Path Profiler. http://www.heywhatsthat.com/profiler.html

32 Shapiro, N. H., Moran, M. J., Fundamentals of Engineering Thermodynamics, $5^{\text {th }}$ edition. 2004.

33 Clark, N. N., Enrique, B. R., McKain, D. L., Victor, P., H., Scott, W., Walter, V., Barnett, R. A., Gautam, M., Thompson, G., Lyons, D. W., Lee, S. "Evaluation of Emissions from New and In-Use Transit Buses in Mexico City, Mexico." Journal of the Transportation Research Board. No. 1987. Washington, DC. 2006: 42-53.

34 Hucho, W. H. Aerodynamics of Road Vehicles, $4^{\text {th }}$ edition. 1998. SAE International.

35 Cha, S., Sloan, R. L., Black, F. M. "Study of Models for the Prediction of Average Road Load of In-Use Delivery Trucks.” SAE Technical Paper. SAE 831799.

36 Environmental Protection Agency: Washington, DC, World Wide Web, http://www.epa.gov. August 12, 2008. Page 86.1229-85.

37 Clark, S. K., Dodge, R. N., A Handbook for Rolling Resistance of Pneumatic Tires. Industrial Development Division. Institute of Science and Technology. The University of Michigan. Ann Arbor 1979.

38 Wang, W. G., Palmer, M. G., Bata, R. M., Clark, N. N., Gautam, M., Lyons, D. W. "Determination of Heavy-Duty Energy Consumption by a Chassis Dynamometer." $\underline{\text { SAE }}$ Technical Paper. SAE 922435. 


\section{Appendix}

Table 20. Eaton Hybrid Vehicle Description

\begin{tabular}{|c|c|}
\hline Vehicle: & USPS 2-Ton Truck \\
\hline Mfr: & Freightliner \\
\hline Year (Vehicle): & 2003 \\
\hline Engine Mfr: & Mercedes Benz 904 \\
\hline Engine ID: & 0904327768 \\
\hline Year (Engine): & 2003 \\
\hline Displacement: & 4.25 liters \\
\hline Aspiration: & Turbocharged \\
\hline Engine Controls: & $\begin{array}{l}\text { ECM, Turbo, Charge Air Cooler, Direct } \\
\text { Injection, Eaton Hybrid }\end{array}$ \\
\hline VIN: & 4UZAANCPX4CL85770 \\
\hline $\begin{array}{l}\begin{array}{l}\text { Vehicle Gross } \\
\text { (GVWR) }\end{array} \\
\text { (GVhicle }\end{array}$ & $14140 \mathrm{lbs}$ \\
\hline Measured Curb Weight (Unloaded) & $10600 \mathrm{lbs}$ \\
\hline Half of Payload $1 / 2 *(G V W R-$ Curb $)$ & $1770 \mathrm{lbs}$ \\
\hline Calculated Half-Payload Test Weight & $12370 \mathrm{lbs}$ \\
\hline
\end{tabular}


Table 21. Baseline (stock) Vehicle Description

\begin{tabular}{|c|c|}
\hline Vehicle: & USPS 2-Ton Truck \\
\hline Mfr: & Freightliner \\
\hline Year (Vehicle): & 2003 \\
\hline Engine Mfr: & Mercedes Benz 904 \\
\hline Engine ID: & 0904298964 \\
\hline Year (Engine): & 2003 \\
\hline Displacement: & 4.25 liters \\
\hline Aspiration: & Turbocharged \\
\hline Engine Controls: & $\begin{array}{l}\text { ECM, Turbo, Charge Air Cooler, Direct } \\
\text { Injection, }\end{array}$ \\
\hline VIN: & 4UZAANCPO3CL84903 \\
\hline $\begin{array}{l}\text { Vehicle Gross Vehicle Weight Rating } \\
\text { (GVWR) }\end{array}$ & $14140 \mathrm{lbs}$ \\
\hline Measured Curb Weight (Unloaded) & $10000 \mathrm{lbs}$ \\
\hline Half of Payload $1 / 2 *(G V W R-$ Curb $)$ & $2070 \mathrm{lbs}$ \\
\hline Calculated Half-Payload Test Weight & $12070 \mathrm{lbs}$ \\
\hline
\end{tabular}




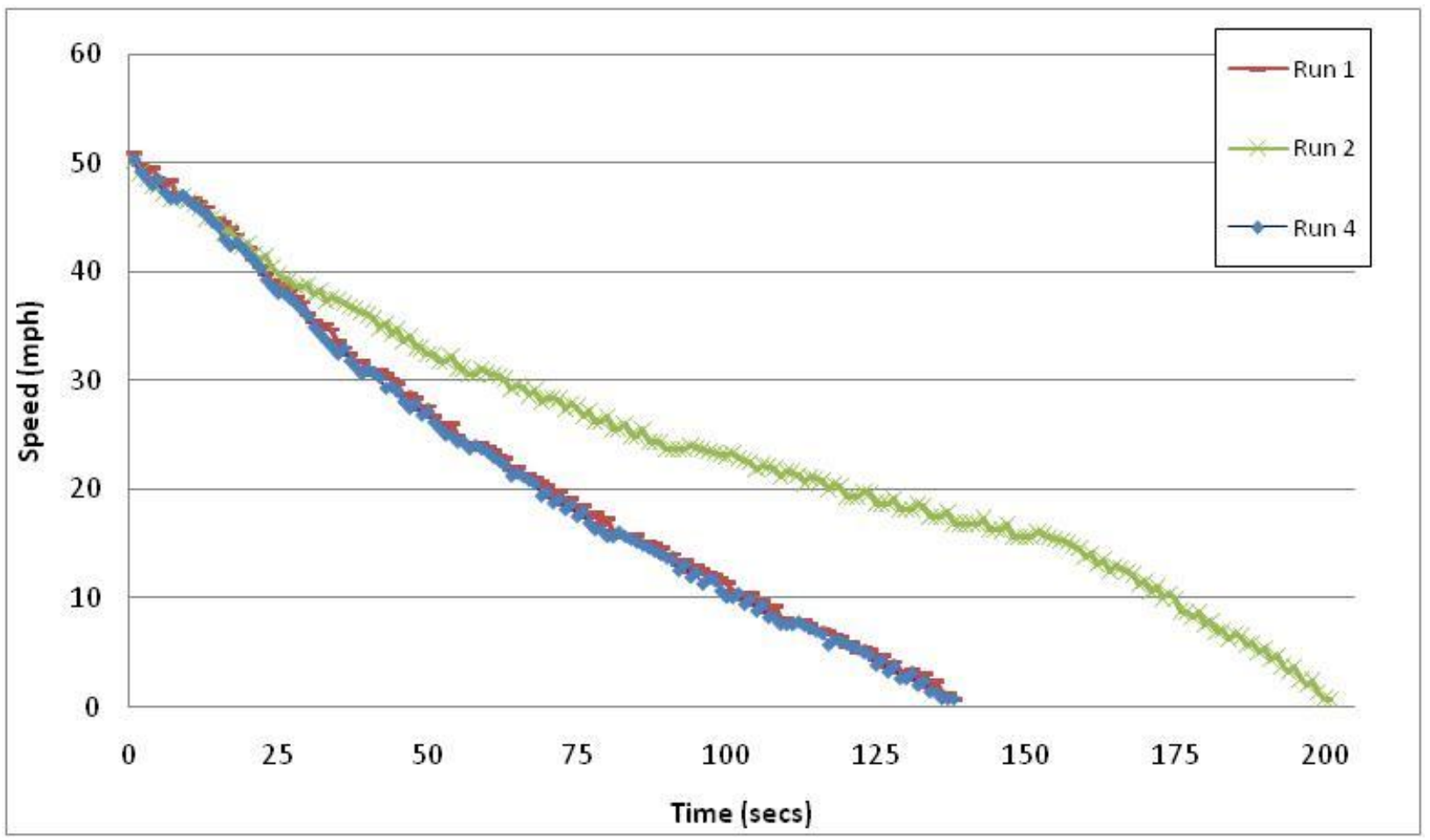

Figure 30. Coastdown comparisons of the hybrid vehicle

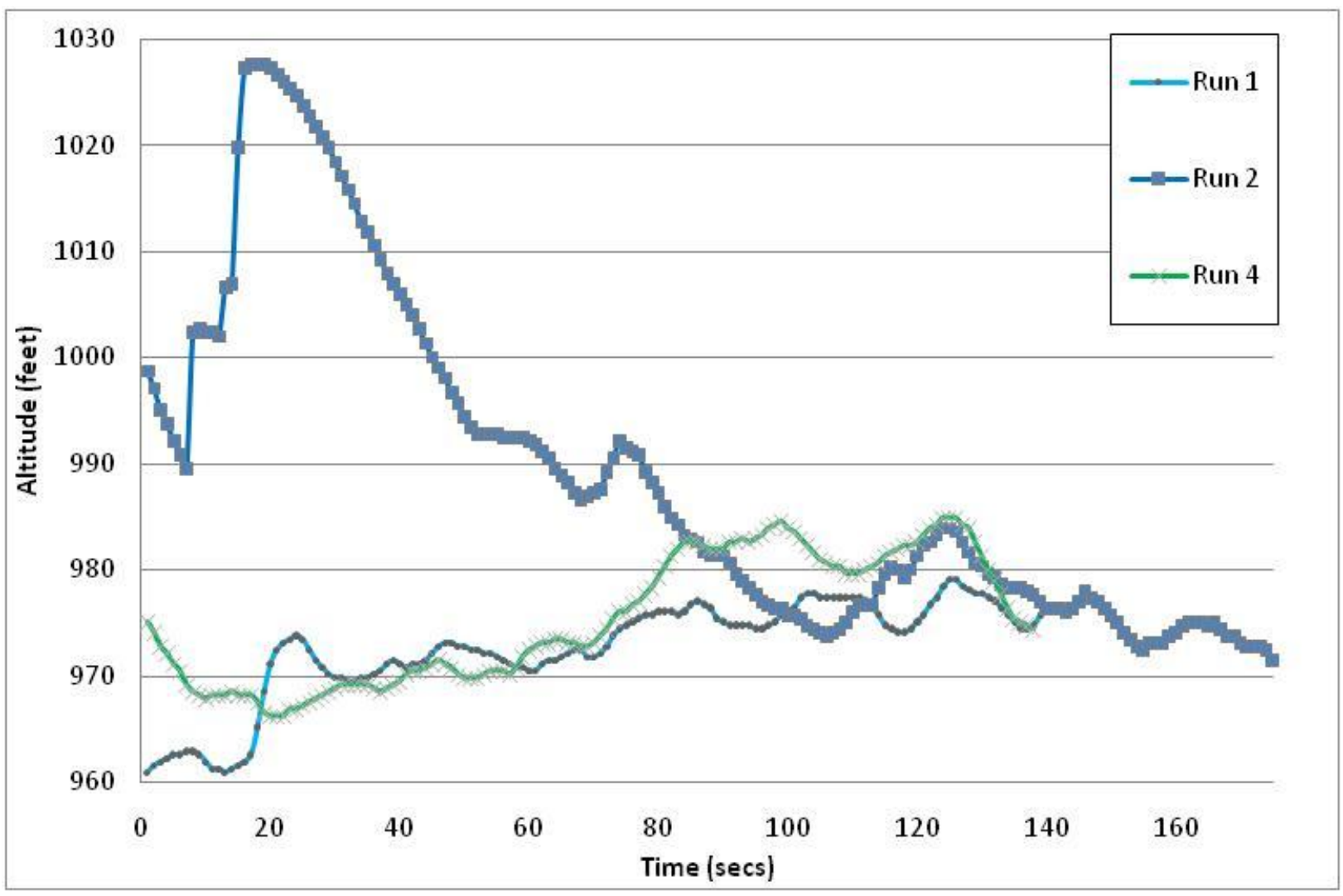

Figure 31 . The elevation change for the hybrid vehicle coastdowns 


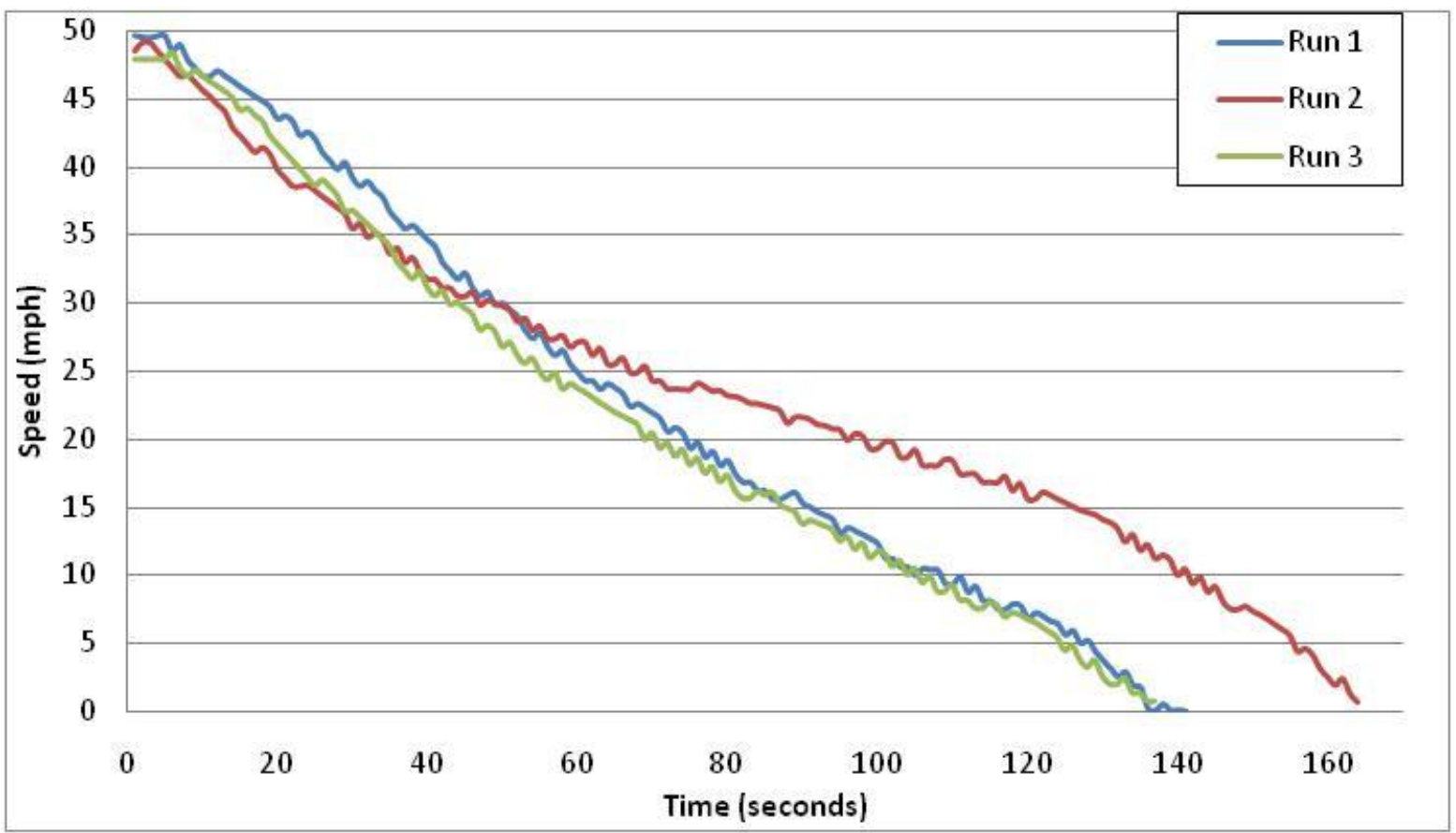

Figure 32. Coastdown comparisons of the unladen baseline vehicle

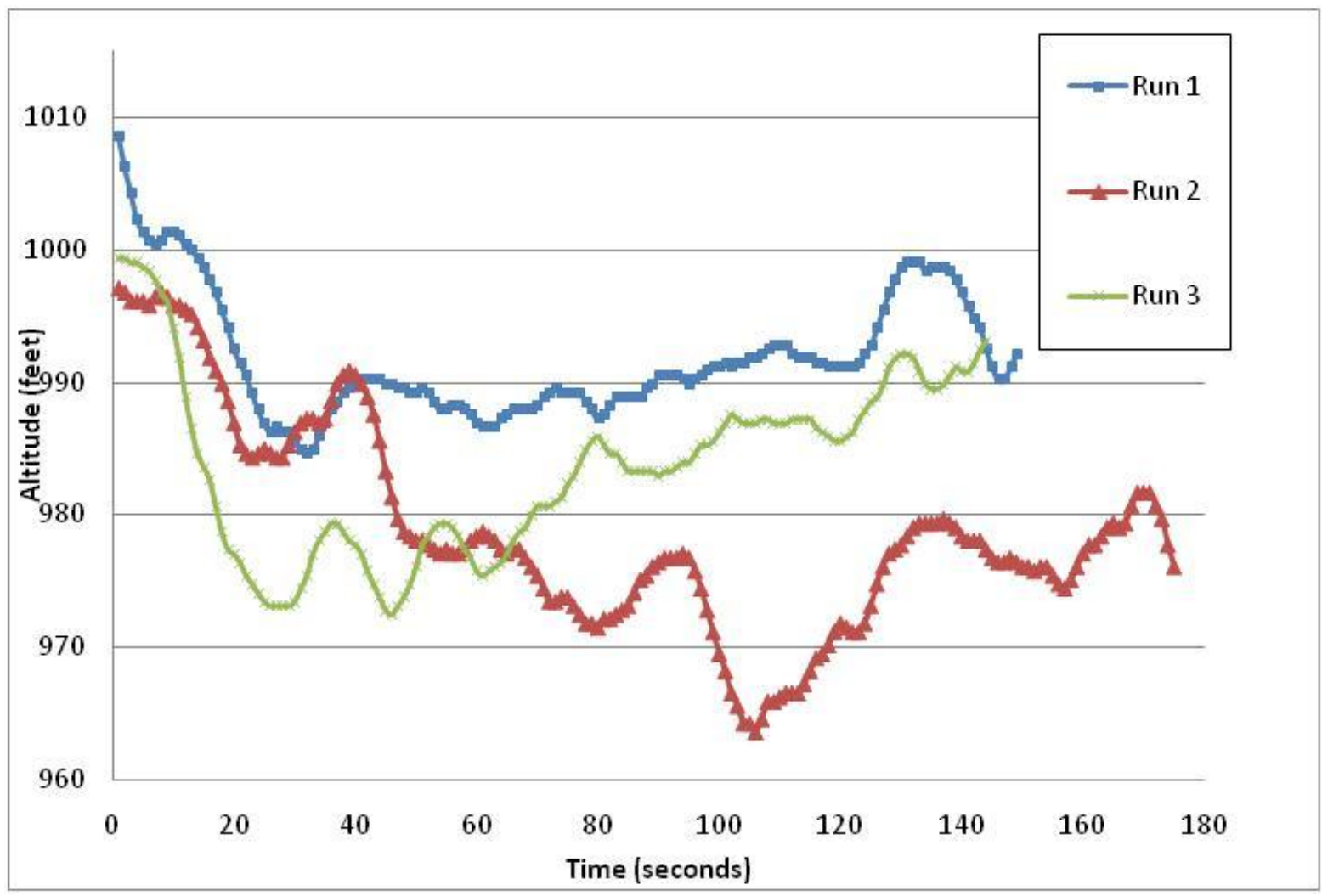

Figure 33. Elevation change for the unladen baseline vehicle for three coastdowns runs 


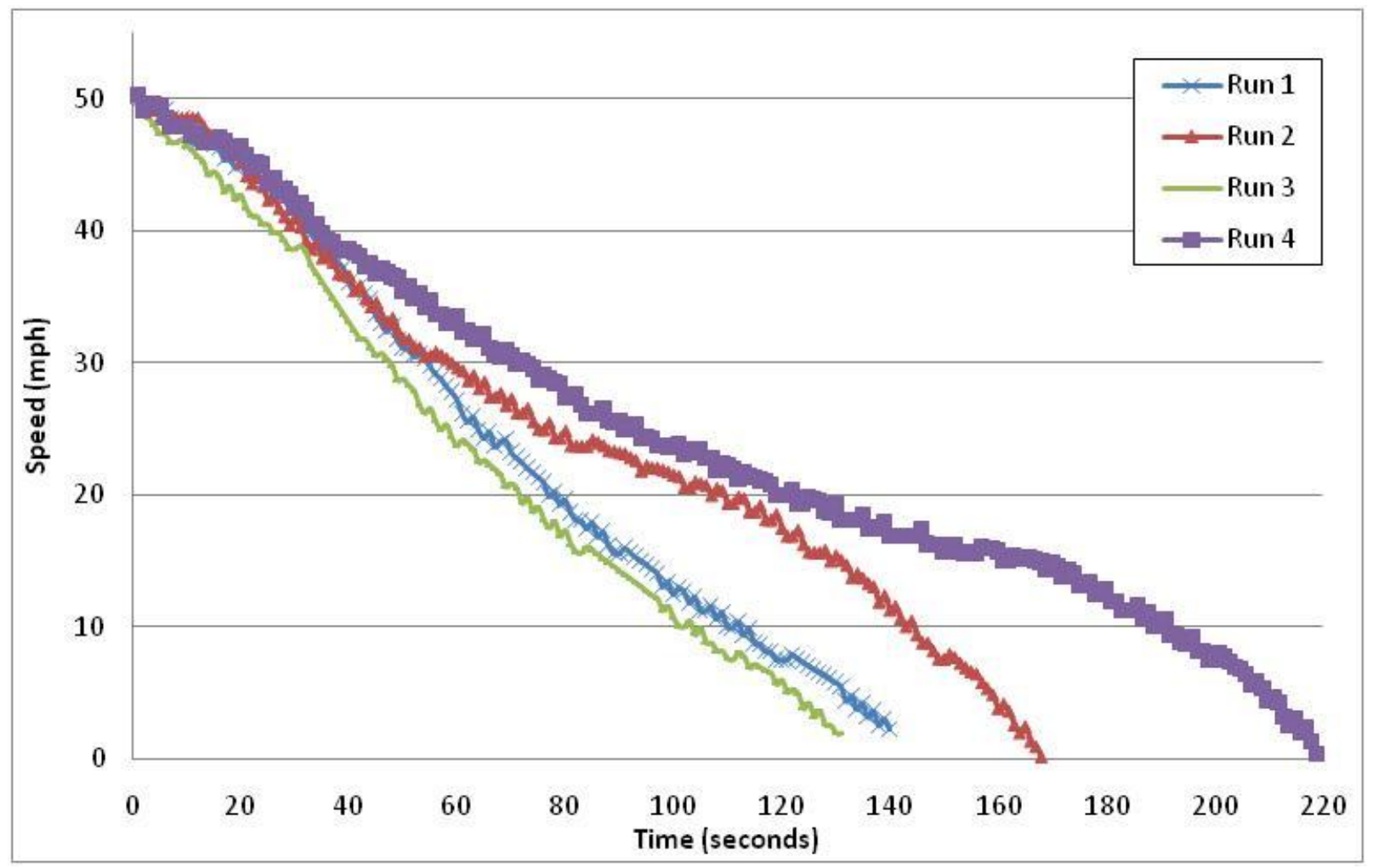

Figure 34. Coastdown comparisons of the loaded baseline vehicle

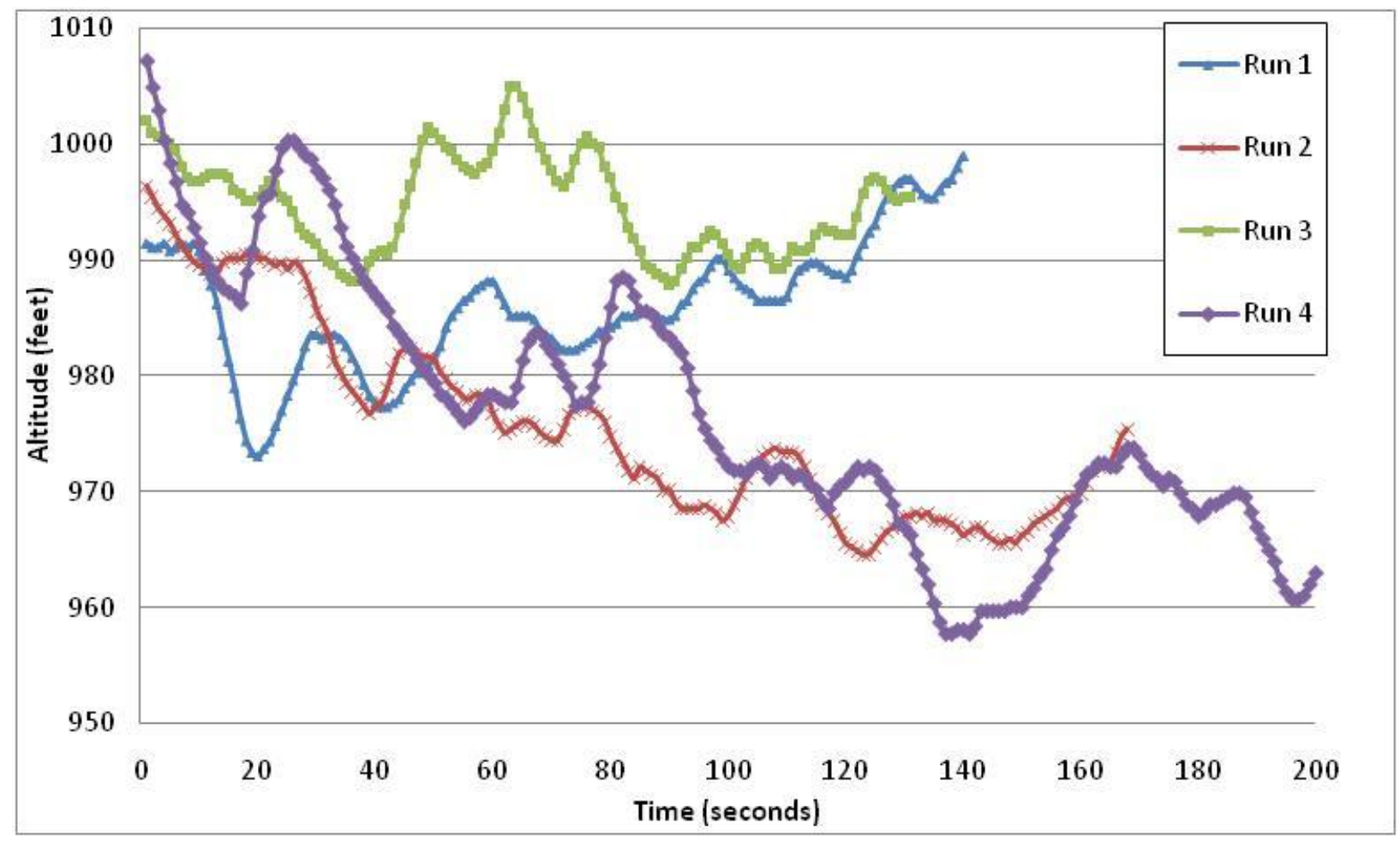

Figure 35. Elevation change for the loaded baseline vehicle for the four runs 
Table 22. Fuel specification from local station

\begin{tabular}{|c|c|c|}
\hline \multicolumn{3}{|c|}{ Fuel Information } \\
\hline SG (60 F) & 0.848 & \\
\hline Water Density $(60 \mathrm{~F})$ & 8.337 & lb/gallon \\
\hline Density (60 F) & 7.072 & lb/gallon \\
\hline
\end{tabular}

Table 23. The coefficient of regression from analysis of speed data from FTP-75 test schedule using the method of theoretical unladen coastdown

\begin{tabular}{|l|c|c|c|c|}
\hline & Slope & Intercept & $\begin{array}{l}\text { Std. } \\
\text { Error }\end{array}$ & $\begin{array}{l}\text { Correlation Coefficient } \\
\left(\mathrm{R}^{2}\right)\end{array}$ \\
\hline Run 1 & 0.991 & 0.026 & 1.04 & 0.996 \\
\hline Run 2 & 0.991 & 0.068 & 0.878 & 0.997 \\
\hline Run 3 & 0.993 & 0.081 & 0.810 & 0.997 \\
\hline
\end{tabular}

Table 24. The coefficient of regression from analysis of speed data from FTP-75 test schedule using the method of theoretical loaded coastdown

\begin{tabular}{|l|c|c|c|c|}
\hline & Slope & Intercept & Std. Error & Correlation Coefficient (R2) \\
\hline Run 1 & 0.981 & 0.188 & 1.27 & 0.993 \\
\hline Run 2 & 0.988 & 0.027 & 0.846 & 0.997 \\
\hline Run 3 & 0.987 & 0.048 & 0.831 & 0.997 \\
\hline
\end{tabular}

Table 25. The coefficient of regression from analysis of speed data from FTP-75 test schedule using the method of empirical loaded coastdown

\begin{tabular}{|l|c|c|c|c|}
\hline & Slope & Intercept & std. Error & Correlation Coefficient $\left(\mathrm{R}^{2}\right)$ \\
\hline Run 1 & 0.993 & 0.487 & 1.20 & 0.994 \\
\hline Run 2 & 0.992 & 0.168 & 0.732 & 0.998 \\
\hline Run 3 & 0.995 & 0.232 & 1.08 & 0.995 \\
\hline
\end{tabular}

Table 26. The coefficient of regression from analysis of speed data from FTP-75 test schedule using the empirical unladed coastdown

\begin{tabular}{|l|c|c|c|c|}
\hline & Slope & Intercept & Std. Error & Correlation Coefficient $\left(\mathrm{R}^{2}\right)$ \\
\hline Run 1 & 0.998 & 0.140 & 0.773 & 0.998 \\
\hline Run 2 & 1.00 & 0.139 & 0.719 & 0.998 \\
\hline Run 3 & 1.00 & 0.155 & 0.727 & 0.998 \\
\hline
\end{tabular}


Table 27. The coefficient of regression from analysis of speed data from FTP-75 test schedule using the method of empirical loaded coastdown

\begin{tabular}{|l|c|c|c|c|}
\hline & Slope & Intercept & Std. Error & Correlation Coefficient $\left(\mathrm{R}^{2}\right)$ \\
\hline Run 32 & 0.987 & -0.016 & 1.34 & 0.993 \\
\hline Run 33 & 0.991 & 0.003 & 1.34 & 0.993 \\
\hline Run 34 & 0.986 & 0.028 & 1.34 & 0.993 \\
\hline
\end{tabular}

Table 28. The coefficient of regression from the analysis of speed data from FTP-75 test schedule using the method of theoretical loaded coastdown

\begin{tabular}{|l|c|c|c|c|}
\hline & Slope & Intercept & Std. Error & Correlation Coefficient (R2) \\
\hline Run 21 & 0.978 & 0.248 & 1.47 & 0.991 \\
\hline Run 22 & 0.978 & 0.243 & 1.47 & 0.991 \\
\hline Run 23 & 0.978 & 0.217 & 1.47 & 0.991 \\
\hline
\end{tabular}

Table 29. The coefficient of regression from the analysis of speed data from FTP-75 test schedule using the method of theoretical unladen coastdown

\begin{tabular}{|l|c|c|c|c|}
\hline & Slope & Intercept & Std. Error & Correlation Coefficient $\left(\mathrm{R}^{2}\right)$ \\
\hline Run 30 & 0.988 & 0.155 & 1.12 & 0.995 \\
\hline Run 31 & 0.988 & 0.105 & 1.13 & 0.995 \\
\hline
\end{tabular}

Table 30. The coefficient of regression from the analysis of power data from the FTP-75 test schedule using the method of theoretical unladed coastdown

\begin{tabular}{|l|c|c|c|c|}
\hline & Slope & Intercept & Std. Error & Correlation Coefficient (R2) \\
\hline Run 30 & 0.998 & 0.026 & 0.558 & 0.998 \\
\hline Run 31 & 0.999 & 0.020 & 0.548 & 0.998 \\
\hline
\end{tabular}

Table 31. The coefficient of regression from the analysis of power data from the FTP-75 test schedule using the method of theoretical loaded coastdown

\begin{tabular}{|l|c|c|c|c|}
\hline & Slope & Intercept & Std. Error & Correlation Coefficient $\left(\mathrm{R}^{2}\right)$ \\
\hline Run 21 & 0.998 & 0.024 & 0.483 & 0.999 \\
\hline Run 22 & 0.998 & 0.021 & 0.498 & 0.999 \\
\hline Run 23 & 0.998 & 0.022 & 0.521 & 0.999 \\
\hline
\end{tabular}


Table 32. The coefficient of regression from the analysis of power data from the FTP-75 test schedule using the method of empirical coastdown

\begin{tabular}{|l|c|c|c|c|}
\hline & Slope & Intercept & Std. Error & Correlation Coefficient (R2) \\
\hline Run 32 & 1.003 & -0.006 & 0.188 & 0.999 \\
\hline Run 33 & 1.002 & -0.002 & 0.273 & 0.998 \\
\hline Run 34 & 0.917 & 0.176 & 1.90 & 0.874 \\
\hline
\end{tabular}

Table 33. The coefficient of regression from the analysis of power data from the FTP-75 test schedule using the method of theoretical loaded coastdown

\begin{tabular}{|l|c|c|c|c|}
\hline & Slope & Intercept & Std. Error & Correlation Coefficient $\left(\mathrm{R}^{2}\right)$ \\
\hline Run 1 & 1.09 & -0.507 & 3.79 & 0.965 \\
\hline Run 2 & 1.07 & -0.537 & 4.66 & 0.946 \\
\hline Run 3 & 1.00 & -0.297 & 5.75 & 0.910 \\
\hline
\end{tabular}

Table 34. The coefficient of regression from the analysis of power data from the FTP-75 test schedule using the method of empirical loaded coastdown

\begin{tabular}{|l|c|c|c|c|}
\hline & Slope & Intercept & Std. Error & Correlation Coefficient $\left(\mathrm{R}^{2}\right)$ \\
\hline Run 1 & 1.02 & -0.061 & 0.496 & 0.991 \\
\hline Run 2 & 1.02 & -0.072 & 0.426 & 0.993 \\
\hline Run 3 & 1.01 & -0.051 & 0.553 & 0.988 \\
\hline
\end{tabular}

Table 35. The coefficient of regression from the analysis of power data from the FTP-75 test schedule theoretical unladed coastdown

\begin{tabular}{|l|c|c|c|c|}
\hline & Slope & Intercept & Std. Error & Correlation Coefficient $\left(\mathrm{R}^{2}\right)$ \\
\hline Run 1 & 1.09 & -0.545 & 4.10 & 0.963 \\
\hline Run 2 & 1.01 & -0.376 & 5.42 & 0.930 \\
\hline Run 3 & 0.993 & -0.387 & 5.93 & 0.914 \\
\hline
\end{tabular}

Table 36. The coefficient of regression from the analysis of power data from the FTP-75 test schedule using the method of empirical unladed coastdown

\begin{tabular}{|l|c|c|c|c|}
\hline & Slope & Intercept & Std. Error & Correlation Coefficient $\left(\mathrm{R}^{2}\right)$ \\
\hline Run 1 & 1.02 & -0.074 & 0.397 & 0.990 \\
\hline Run 2 & 1.02 & -0.084 & 0.407 & 0.990 \\
\hline Run 3 & 1.02 & -0.087 & 0.408 & 0.990 \\
\hline
\end{tabular}


Table 37. FTP-75 test results from the baseline vehicle using the loaded empirical coastdown

\begin{tabular}{|l|l|l|c|c|c|c|c|}
\hline Test & Run & $\begin{array}{l}\text { Distance } \\
\text { Traveled (mi) }\end{array}$ & $\begin{array}{l}\text { Volume } \\
\text { (gallons) }\end{array}$ & MPG & Work/Hr & $\begin{array}{l}\text { Work } \\
\text { (whphr) }\end{array}$ & $\begin{array}{l}\text { Fuel / Work } \\
\text { (lbs/whphr) }\end{array}$ \\
\hline 4 & 1 & 11.2 & 1.26 & 8.89 & 18.11 & 9.43 & 0.492 \\
\hline 4 & 2 & 11.1 & 1.22 & 9.07 & 18.0 & 9.36 & 0.481 \\
\hline 4 & 3 & 11.0 & 1.28 & 8.63 & 18.2 & 9.45 & 0.498 \\
\hline average & & 11.1 & 1.25 & 8.86 & 18.1 & 9.41 & 0.490 \\
\hline std & & 0.087 & 0.029 & 0.224 & 0.094 & 0.049 & 0.009 \\
\hline COV \% & & 0.784 & 2.28 & 2.53 & 0.518 & 0.518 & 1.77 \\
\hline
\end{tabular}

Table 38. FTP-75 test results from the baseline vehicle using the unladen empirical coastdown

\begin{tabular}{|l|l|l|c|c|c|c|c|}
\hline Test & Run & $\begin{array}{l}\text { Distance } \\
\text { Traveled (mi) }\end{array}$ & $\begin{array}{l}\text { Volume } \\
\text { (gallons) }\end{array}$ & MPG & Work/Hr & $\begin{array}{l}\text { Work } \\
\text { (whphr) }\end{array}$ & $\begin{array}{l}\text { Fuel / Work } \\
\text { (lbs/whphr) }\end{array}$ \\
\hline 3 & 1 & 11.1 & 0.967 & 11.5 & 14.7 & 7.64 & 0.466 \\
\hline 3 & 2 & 11.1 & 0.910 & 12.2 & 15.0 & 7.78 & 0.431 \\
\hline 3 & 3 & 11.1 & 0.910 & 12.2 & 14.86 & 7.74 & 0.433 \\
\hline average & & 11.1 & 0.929 & 12.0 & 14.8 & 7.72 & 0.443 \\
\hline std & & 0.027 & 0.032 & 0.436 & 0.141 & 0.074 & 0.020 \\
\hline COV \% & & 0.243 & 3.49 & 3.64 & 0.954 & 0.954 & 4.42 \\
\hline
\end{tabular}

Table 39. FTP-75 test results from the baseline vehicle using the loaded theoretical coastdown

\begin{tabular}{|l|l|l|l|c|c|c|c|}
\hline Test & Run & $\begin{array}{l}\text { Distance } \\
\text { Traveled (mi) }\end{array}$ & $\begin{array}{l}\text { Volume } \\
\text { (gallons) }\end{array}$ & MPG & Work/Hr & $\begin{array}{l}\text { Work } \\
\text { (whphr) }\end{array}$ & $\begin{array}{l}\text { Fuel / Work } \\
\text { (lbs/whphr) }\end{array}$ \\
\hline 5 & 1 & 10.9 & 1.15 & 9.46 & 22.1 & 11.5 & 0.370 \\
\hline 5 & 2 & 10.9 & 1.20 & 9.12 & 22.6 & 11.7 & 0.375 \\
\hline 5 & 3 & 10.9 & 1.16 & 9.41 & 22.0 & 11.4 & 0.374 \\
\hline average & & 10.9 & 1.17 & 9.33 & 22.2 & 11.6 & 0.373 \\
\hline std & & 0.004 & 0.024 & 0.185 & 0.327 & 0.170 & 0.003 \\
\hline COV \% & & 0.033 & 2.02 & 1.99 & 1.47 & 1.47 & 0.824 \\
\hline
\end{tabular}


Table 40. FTP-75 test results from the baseline vehicle using the unladen theoretical coastdown

\begin{tabular}{|l|l|c|c|c|c|c|c|}
\hline Test & Run & $\begin{array}{l}\text { Distance } \\
\text { Traveled (mi) }\end{array}$ & $\begin{array}{l}\text { Volume } \\
\text { (gallons) }\end{array}$ & MPG & Work/Hr & $\begin{array}{l}\text { Work } \\
\text { (whphr) }\end{array}$ & $\begin{array}{l}\text { Fuel / Work } \\
\text { (lbs/whphr) }\end{array}$ \\
\hline 2 & 1 & 10.8 & 1.13 & 9.54 & 21.2 & 11.1 & 0.376 \\
\hline 2 & 2 & 10.9 & 1.09 & 10.0 & 20.4 & 10.6 & 0.376 \\
\hline 2 & 3 & 10.6 & 1.09 & 9.75 & 20.4 & 10.6 & 0.376 \\
\hline average & & 10.7 & 1.10 & 9.76 & 20.7 & 10.8 & 0.376 \\
\hline std & & 0.139 & 0.025 & 0.227 & 0.462 & 0.241 & 0.000 \\
\hline COV \% & & 1.29 & 2.29 & 2.32 & 2.23 & 2.23 & 0.074 \\
\hline
\end{tabular}

Table 41. FTP-75 test results from the hybrid vehicle using the loaded empirical coastdown

\begin{tabular}{|l|l|l|c|c|c|c|c|}
\hline Test & Run & $\begin{array}{l}\text { Distance } \\
\text { Traveled (mi) }\end{array}$ & $\begin{array}{l}\text { Volume } \\
\text { (gallons) }\end{array}$ & MPG & Work/Hr & $\begin{array}{l}\text { Work } \\
\text { (whphr) }\end{array}$ & $\begin{array}{l}\text { Fuel / Work } \\
\text { (lbs/whphr) }\end{array}$ \\
\hline 8 & 32 & 10.9 & 0.823 & 13.2 & 17.3 & 9.01 & 0.646 \\
\hline 8 & 33 & 10.9 & 0.804 & 13.6 & 17.4 & 9.08 & 0.627 \\
\hline 8 & 34 & 10.9 & 0.792 & 13.8 & 17.3 & 9.01 & 0.622 \\
\hline average & & 10.9 & 0.807 & 13.5 & 17.4 & 9.03 & 0.632 \\
\hline std & & 0.026 & 0.016 & 0.274 & 0.074 & 0.038 & 0.013 \\
\hline COV (\%) & & 0.234 & 1.95 & 2.03 & 0.425 & 0.425 & 2.03 \\
\hline
\end{tabular}

Table 42. FTP-75 test results from the hybrid vehicle using the unladen theoretical coastdown

\begin{tabular}{|l|l|l|c|c|c|c|c|}
\hline Test & Run & $\begin{array}{l}\text { Distance } \\
\text { Traveled (mi) }\end{array}$ & $\begin{array}{l}\text { Volume } \\
\text { (gallons) }\end{array}$ & MPG & Work/Hr & $\begin{array}{l}\text { Work } \\
\text { (whphr) }\end{array}$ & $\begin{array}{l}\text { Fuel / Work } \\
\text { (lbs/whphr) }\end{array}$ \\
\hline 7 & 30 & 11.0 & 0.811 & 13.5 & 17.8 & 9.29 & 0.617 \\
\hline 7 & 31 & 11.0 & 0.811 & 13.5 & 17.7 & 9.20 & 0.623 \\
\hline average & & 11.0 & 0.811 & 13.5 & 17.8 & 9.24 & 0.620 \\
\hline std & & 0.018 & 0.000 & 0.022 & 0.122 & 0.063 & 0.004 \\
\hline COV (\%) & 0.163 & 0.000 & 0.163 & 0.685 & 0.685 & 0.685 \\
\hline
\end{tabular}


Table 43. FTP-75 results from the hybrid vehicle using the loaded theoretical coastdown

\begin{tabular}{|l|l|c|c|c|c|c|c|}
\hline Test & Run & $\begin{array}{l}\text { Distance } \\
\text { Traveled (mi) }\end{array}$ & $\begin{array}{l}\text { Volume } \\
\text { (gallons) }\end{array}$ & MPG & Work/Hr & $\begin{array}{l}\text { Work } \\
\text { (whph) }\end{array}$ & $\begin{array}{l}\text { Fuel / Work } \\
\text { (lbs/whphr) }\end{array}$ \\
\hline 3 & 21 & 10.9 & 0.879 & 12.4 & 19.3 & 10.1 & 0.618 \\
\hline 4 & 22 & 10.9 & 0.886 & 12.3 & 19.3 & 10.1 & 0.623 \\
\hline 4 & 23 & 10.9 & 0.873 & 12.5 & 19.3 & 10.1 & 0.614 \\
\hline average & & 10.9 & 0.879 & 12.4 & 19.3 & 10.1 & 0.618 \\
\hline std & & 0.008 & 0.006 & 0.084 & 0.002 & 0.001 & 0.004 \\
\hline COV (\%) & & 0.077 & 0.709 & 0.676 & 0.010 & 0.010 & 0.704 \\
\hline
\end{tabular}

